TRANSACTIONS OF THE

AMERICAN MATHEMATICAL SOCIETY

Volume 363, Number 2, February 2011, Pages 801-856

S 0002-9947(2010)05145-1

Article electronically published on September 30, 2010

\title{
LOCAL OPERATOR ALGEBRAS, FRACTIONAL POSITIVITY AND THE QUANTUM MOMENT PROBLEM
}

\author{
ANAR DOSI
}

\begin{abstract}
In the present paper we introduce quantum measures as a concept of quantum functional analysis and develop the fractional space technique in the quantum (or local operator) space framework. We prove that each local operator algebra (or quantum *-algebra) has a fractional space realization. This approach allows us to formulate and prove a noncommutative AlbrechtVasilescu extension theorem, which in turn solves the quantum moment problem.
\end{abstract}

\section{INTRODUCTION}

The paper deals with the quantum, or quantized measures as a concept of local operator space (or quantum space) theory [26, [12, [9]. Quantum spaces play the role of locally convex spaces in quantum functional analysis [11, 14] (see also [16]). They unify various mathematical structures such as unbounded operators on a Hilbert space, multinormed $C^{*}$-algebras and locally convex spaces under the same matrix framework 9]. Thus each quantum space or local operator space can be realized as a subspace of the multinormed $C^{*}$-algebra of unbounded operators with its canonical quantum (or matrix) topology [7, whereas a normed quantum space or operator space is identified [12, 2.3.5] with a subspace in the $C^{*}$-algebra $\mathcal{B}(H)$ of all bounded linear operators on a Hilbert space $H$.

One of the fundamental concepts of classical functional analysis is that of spectral measure, which is the basic tool in the operator moment problem. The problem is to find a positive operator-valued measure which expresses an integral representation for the given unital linear mapping from the algebra of all complex-valued polynomials in several real variables into the space of all sesquilinear forms on a pre-Hilbert space. Namely, fix an inner-product space $\Delta$ whose completion is the Hilbert space $K, S F(\Delta)$ is a linear space of all sesquilinear forms on $\Delta$, and let $\mathcal{P}_{t}$ be an algebra of all complex-valued polynomial functions in $n$-real variables $t=\left(t_{1}, \ldots, t_{n}\right)$. The operator moment problem 23] with respect to a fixed unital linear mapping (or distribution) $\phi: \mathcal{P}_{t} \rightarrow S F(\Delta)$ is to find a positive measure $F$

Received by the editors November 11, 2008 and, in revised form, April 1, 2009.

2000 Mathematics Subject Classification. Primary 47L60; Secondary 47L25, 46L07, 46G12.

Key words and phrases. Local operator algebra, quantum system, quantum moment problem, fractional space, fractional positivity. 
over all Borel subsets of $\mathbb{R}^{n}$ with the operator values in $\mathcal{B}(K)$ such that

$$
\phi(p(t))(x, x)=\int p(t) d F_{x, x}
$$

for all $p(t) \in \mathcal{P}_{t}$ and $x \in \Delta$. In this case the mapping $\phi: \mathcal{P}_{t} \rightarrow S F(\Delta)$ is called a moment form and $F$ is a representing measure for $\phi$. If the measure $F$ is focusing on a fixed closed set $L \subseteq \mathbb{R}^{n}$, then we say that $\phi$ is an $L$-moment form. Since an operator-valued positive measure $F$ might be treated as a unital (matrix) positive linear mapping $\Psi: C(\Omega) \rightarrow \mathcal{B}(K), \Psi(f)=\int_{\Omega} f d F$, on the algebra of all complex continuous functions $C(\Omega)$ over some compact topological space $\Omega$, the operator moment problem can be thought of as a matrix positive extension problem of the original mapping. Consequently, this problem has purely quantum nature; just recall Arveson-Wittstock type extension theorems [12, 26]. The main tool in this approach uses the fractional space machinery developed in 2. by Albrecht and Vasilescu, and in [24, 25] by Vasilescu. Considering $\mathcal{P}_{t}$ as a fractional subspace of the fractional space associated by the commutative $C^{*}$ algebra $C\left(\left(\mathbb{R}_{\infty}\right)^{n}\right)$, the original "positive" mapping $\phi: \mathcal{P}_{t} \rightarrow S F(\Delta)$ is extending up to a "positive" mapping over the fractional space, which in turn generates the required positive mapping $\Psi: C\left(\left(\mathbb{R}_{\infty}\right)^{n}\right) \rightarrow \mathcal{B}(K)$, where $\mathbb{R}_{\infty}=\mathbb{R} \cup\{\infty\}$ is the point compactification of the real line $\mathbb{R}$. Replacing $\mathbb{R}^{n}$ by its closed subset $L$ and $\left(\mathbb{R}_{\infty}\right)^{n}$ by the closure of $L$ in $\left(\mathbb{R}_{\infty}\right)^{n}$, the operator moment problem has a solution in a similar manner, just demanding the original mapping $\phi: \mathcal{P}_{t} \rightarrow S F(\Delta)$ to be $L$-compatible in the sense that $\phi(p(t))=0$ whenever $p(L)=\{0\}$.

A simple quantum review (see [8]) of the considered real variables $t_{1}, \ldots, t_{n}$ amounts to the quantum measures, which is the central concept of the present manuscript. We are replacing the complex-valued polynomial functions in several real variables $t_{1}, \ldots, t_{n}$ by elements of a local operator algebra (or quantum *-algebra) generated by several symmetric unbounded operators $T_{1}, \ldots, T_{n}$ on a Hilbert space. The quantum moment problem asks whether a given distribution over the polynomial algebra $\mathcal{P}_{T}$ would determine a quantum measure. This general form of the quantum moment problem can be used in various applications (see [5], [18, 1]). The fractional space construction has been quantized in terms of the quantum (or local operator) systems (see [9]). We prove that each local operator algebra (or quantum *-algebra) is a fractional subspace of the multinormed $C^{*}$ algebra of all noncommutative continuous functions on a quantum domain. The quantum measures are introduced as matrix positive mappings over the quantized fractional space. In particular, the operator moment problem turns out to be a "functional version" of the quantum one. Based upon a locally convex version of the Arveson-Wittstock theorem [9] and the fractional space technique, we derive the existence of a quantum measure that would lead to the solution of the quantum moment problem. The crucial role in this approach plays the fractional positivity. To be precise, let us present some details.

Fix a Hilbert space $H$ with its upward filtered family $\mathcal{E}=\left\{H_{\alpha}\right\}$ of closed subspaces whose union $\mathcal{D}$ is a dense subspace. So we have a net $\mathfrak{p}=\left\{P_{\alpha}\right\}$ of projections in $\mathcal{B}(H)$ such that $\lim \mathfrak{p}=1_{H}$ SOT. The space $\mathcal{D}$ can be thought of as a quantization of the space $\mathbb{R}^{n}$ (which is the union of its directed compact subsets), whereas $H$ plays the role of $\left(\mathbb{R}_{\infty}\right)^{n}$. By the $*$-algebra $C_{\mathcal{E}}^{*}(\mathcal{D})$ of all "noncommutative continuous functions" over the domain $\mathcal{E}$ with its union space $\mathcal{D}[9$, we mean the set of those linear transformations $T: \mathcal{D} \rightarrow \mathcal{D}$ such that $P_{\alpha} T \subseteq T P_{\alpha}$ for all $\alpha \in \Lambda$. Thus 
each $T \in C_{\mathcal{E}}^{*}(\mathcal{D})$ being an unbounded operator on $H$ with the domain $\operatorname{dom}(T)=\mathcal{D}$ admits an unbounded dual $T^{\star}$ such that $\mathcal{D} \subseteq \operatorname{dom}\left(T^{\star}\right)$ and $T^{*}=T^{\star} \mid \mathcal{D} \in C_{\mathcal{E}}^{*}(\mathcal{D})$. The correspondence $T \mapsto T^{*}$ is an involution in $C_{\mathcal{E}}^{*}(\mathcal{D})$, and we have a family of $C^{*}$-seminorms

$$
\|T\|_{\alpha}=\left\|T \mid H_{\alpha}\right\|_{\mathcal{B}\left(H_{\alpha}\right)}, \quad T \in C_{\mathcal{E}}^{*}(\mathcal{D}), \quad \alpha \in \Lambda .
$$

It can be proved that $C_{\mathcal{E}}^{*}(\mathcal{D})$ turns out to be a unital multinormed $C^{*}$-algebra with respect to the indicated involution and the family of seminorms (see [9]). In particular, it possesses a canonical quantum space structure (see [9]). By a quantum *-algebra we mean any $*$-subalgebra in $C_{\mathcal{E}}^{*}(\mathcal{D})$. We also introduce the $C^{*}$-algebra $C_{\mathcal{E}}^{*}(H)$ of all bounded operators $T \in \mathcal{B}(H)$ such that $T P_{\alpha}=P_{\alpha} T$ for all $\alpha$; that is, $C_{\mathcal{E}}^{*}(H)=\mathfrak{p}^{\prime}$ is the commutant of $\mathfrak{p}$ in $\mathcal{B}(H)$. The algebras $C_{\mathcal{E}}(\mathcal{D})$ and $C_{\mathcal{E}}^{*}(H)$ are the quantized (or noncommutative) versions of $C\left(\mathbb{R}^{n}\right)$ and $C\left(\left(\mathbb{R}_{\infty}\right)^{n}\right)$, respectively. We mention that $C_{\mathcal{E}}^{*}(H)$ can be identified with a dense subalgebra in $C_{\mathcal{E}}^{*}(\mathcal{D})$ (see Section 5) by means of the restriction mapping $T \mapsto T \mid \mathcal{D}$.

A self-adjoint element $m \in C_{\mathcal{E}}^{*}(H)$ is said to be a denominator if it is an $\mathcal{E}$ bijection; that is, $m \mid H_{\alpha}$ is invertible in $\mathcal{B}\left(H_{\alpha}\right)$ for all $\alpha$. The relevant fractional space is defined as

$$
C_{\mathcal{E}}^{*}(H) / m=\left\{T / m: T \in C_{\mathcal{E}}^{*}(H)\right\},
$$

where $T / m=(T \mid \mathcal{D})(m \mid \mathcal{D})^{-1}$ is an unbounded operator on $H$. Actually, $C_{\mathcal{E}}^{*}(H) / m$ is a dense subspace in $C_{\mathcal{E}}^{*}(\mathcal{D})$ (Section 5 ). Moreover, it possesses a canonical operator space (or normed quantum space) structure derived from the $C^{*}$-algebra $C_{\mathcal{E}}^{*}(H)$. For a (unital) subset $M \subseteq C_{\mathcal{E}}^{*}(H)$ of denominators the relevant fractional space is denoted by $C_{\mathcal{E}}^{*}(H) / M$. Thus

$$
C_{\mathcal{E}}^{*}(H) / M=\sum_{m \in M} C_{\mathcal{E}}^{*}(H) / m \subseteq C_{\mathcal{E}}^{*}(\mathcal{D}) .
$$

Replacing $C_{\mathcal{E}}^{*}(H)$ by some $C^{*}$-subalgebra $\mathfrak{J}_{\mathcal{E}}$, one may introduce a fractional space $\mathfrak{J}_{\mathcal{E}} / M$ in a similar manner (see Definition [5.2). Being an inductive limit of the normed operator spaces, the fractional space $\mathfrak{J}_{\mathcal{E}} / M$ turns out to be a quantum space. The fractionality is preserved over all matrix spaces $\mathbb{M}_{k}(\mathfrak{J} \mathcal{E} / M), k \in \mathbb{N}$. Namely, for each $k$ we have

$$
\mathbb{M}_{k}\left(\mathfrak{J}_{\mathcal{E}} / M\right)=\sum_{m \in M} \mathbb{M}_{k}\left(\mathfrak{J}_{\mathcal{E}}\right) /\left(m \cdot 1_{H^{k}}\right)=\mathbb{M}_{k}\left(\mathfrak{J}_{\mathcal{E}}\right) /\left(M \cdot 1_{H^{k}}\right) .
$$

Using the Cayley transform technique, one may derive that each quantum $*$-algebra $V \subseteq C_{\mathcal{E}}^{*}(\mathcal{D})$ is a fractional space. In particular,

$$
C_{\mathcal{E}}^{*}(\mathcal{D})=\sum_{m} C_{\mathcal{E}}^{*}(H) / m
$$

Each fractional space $\mathfrak{J}_{\mathcal{E}} / M$ has the cone $\left(\mathfrak{J}_{\mathcal{E}} / M\right)^{+}$of positive elements. Namely, an element $b \in \mathfrak{J}_{\mathcal{E}} / m$ is said to be fractionally positive if $b=\sum_{i=1}^{k} T_{i} / n_{i}$ for some $T_{i} \in \mathfrak{J}_{\mathcal{E}}, n_{i} \in M$, such that all $T_{i} \geq 0$ and $n_{i}^{-1} m$ are bounded. Similarly, we have the cones $\mathbb{M}_{k}\left(\mathfrak{J}_{\mathcal{E}} / M\right)^{+}, k \in \mathbb{N}$. This positivity strongly depends on the choice of the denominator set $M$. Further, let $\Delta$ be an inner product space whose completion is the Hilbert space $K$. By a quantum $\mathcal{B}(K)$-valued measure on $\mathfrak{J}_{\mathcal{E}}$ (with respect to $M)$ we mean a unital matrix positive linear mapping $\Psi: \mathfrak{J}_{\mathcal{E}} \rightarrow \mathcal{B}(K)$ which has a 
matrix positive sesquilinear extension $\mu: \mathfrak{J}_{M} / M \rightarrow S F(\Delta)$. Namely, assume that the functionals

$$
\mu_{x, y}: \mathfrak{J}_{\mathcal{E}} \rightarrow \mathbb{C}, \quad \mu_{x, y}(T)=\langle\Psi(T) x, y\rangle
$$

have linear extensions $\widetilde{\mu}_{x, y}: \mathfrak{J}_{\mathcal{E}} / M \rightarrow \mathbb{C}, x, y \in \Delta$, such that the mapping $\Delta \times \Delta \rightarrow$ $\mathbb{C},(x, y) \mapsto \widetilde{\mu}_{x, y}(b)$ is a sesquilinear form on $\Delta$ for each $b \in \mathfrak{J}_{M} / M$. Then we say that the linear mapping

$$
\mu: \mathfrak{J}_{M} / M \rightarrow S F(\Delta), \quad \mu(b)(x, y)=\widetilde{\mu}_{x, y}(b)
$$

is a sesquilinear extension of $\Psi$. Further, the mapping $\mu$ is matrix positive if for each $k \in \mathbb{N}$ and $x=\left[x_{i}\right] \in \Delta^{k}$ the mapping

$$
\mu_{x}^{[k]}: \mathbb{M}_{k}\left(\mathfrak{J}_{M} / M\right) \rightarrow \mathbb{M}_{k^{2}}, \quad \mu_{x}^{[k]}(b)=\left[\begin{array}{lll}
\tilde{\mu}_{x_{1}, x_{1}}^{(k)}(b) & \cdots & \widetilde{\mu}_{x_{k}, x_{1}}^{(k)}(b) \\
\vdots & & \vdots \\
\tilde{\mu}_{x_{1}, x_{k}}^{(k)}(b) & \cdots & \widetilde{\mu}_{x_{k}, x_{k}}^{(k)}(b)
\end{array}\right]
$$

is fractionally positive. We briefly say that $\Psi: \mathfrak{J}_{\mathcal{E}} \rightarrow \mathcal{B}(K)$ admits a sesquilinear extension $\mu: \mathfrak{J}_{M} / M \rightarrow S F(\Delta)$ which is fractionally matrix positive.

Now let us consider mutually commuting symmetric operators $T=\left(T_{1}, \ldots, T_{n}\right)$ in $C_{\mathcal{E}}^{*}(\mathcal{D})$, that is, $T_{i}=T_{i}^{*}$ for all $i$, instead of the real variables $t=\left(t_{1}, \ldots, t_{n}\right)$ in the classical operator moment problem. They are quantum variables or operators of observables. The unital associative subalgebra in $C_{\mathcal{E}}^{*}(\mathcal{D})$ generated by $T$ is denoted by $\mathcal{P}_{T}$. Obviously, $\mathcal{P}_{T}$ is a quantum $*$-algebra in $C_{\mathcal{E}}^{*}(\mathcal{D})$. The tuple $T$ generates a set of positive denominators

$$
M=\left\{\left(1+T_{1}^{2}\right)^{-\lambda_{1}} \cdots\left(1+T_{n}^{2}\right)^{-\lambda_{n}}: \lambda=\left(\lambda_{1}, \ldots, \lambda_{n}\right) \in \mathbb{Z}_{+}^{n}\right\}
$$

in $C_{\mathcal{E}}^{*}(H)$. Let $M_{\mathcal{E}}^{\prime}$ be the commutant in $C_{\mathcal{E}}^{*}(H)$ of the set $M$, which is a unital (noncommutative) $C^{*}$-algebra. So, we have a fractional space $M_{\mathcal{E}}^{\prime} / M$. The algebra $M_{\mathcal{E}}^{\prime}$ plays the role of $C\left(\left(\mathbb{R}_{\infty}\right)^{n}\right)$. Moreover, $\mathcal{P}_{T}$ is a fractional subspace in $M_{\mathcal{E}}^{\prime} / M$. Thus the quantum moment problem with respect to a fixed unital linear mapping $\phi: \mathcal{P}_{T} \rightarrow S F(\Delta)$ is to find a quantum $\mathcal{B}(K)$-valued measure $\mu$ on the $C^{*}$-algebra $M_{\mathcal{E}}^{\prime}$ such that

$$
\phi(p(T))(x, x)=\widetilde{\mu}_{x}(p(T))
$$

for all $p(T) \in \mathcal{P}_{T}$ and $x \in \Delta$. In this case the mapping $\phi: \mathcal{P}_{T} \rightarrow S F(\Delta)$ is called a moment form and $\mu$ is a representing quantum measure for $\phi$.

The main result (see Theorem 7.3) of the paper is the noncommutative AlbrechtVasilescu extension theorem (see [2, Theorem 2.5] for the functional case), where a matrix contractive inner-product mapping $\phi: \mathcal{F} \rightarrow S F(\Delta)$ on a fractional subspace $\mathcal{F} \subseteq \mathfrak{J}_{M} / M$ is extended up to a unital matrix positive mapping $\psi: \mathfrak{J}_{M} / M \rightarrow$ $S F(\Delta)$ with the required properties. Each such extension produces a quantized measure on $\mathfrak{J}_{M}$ automatically (see Proposition 7.2 ) and solves the quantum moment problem.

It is worth noting that the classical functional scheme of the construction can be thought of as a particular case of the quantized one. Indeed, set $H=L^{2}\left(\mathbb{R}^{n}\right)$ and $H_{\alpha}=\left\{f \in H: \operatorname{supp}(f) \subseteq K_{\alpha}\right\}$, where $\left\{K_{\alpha}\right\}$ is an upward filtered (countable) family of a compact subset in $\mathbb{R}^{n}$ such that $\mathbb{R}^{n}=\bigcup_{\alpha} K_{\alpha}$. The real variable $t_{k}$ can be identified with the unbounded multiplication operators on $H$. Then $t_{k}\left(H_{\alpha}\right) \subseteq H_{\alpha}$ and $t_{k} \mid H_{\alpha} \in \mathcal{B}\left(H_{\alpha}\right)$ for all $k$ and $\alpha$. Thus the algebra $\mathcal{P}_{t}$ of all polynomial functions turns out to be a quantum $*$-algebra in the algebra $C_{\mathcal{E}}^{*}(\mathcal{D})$ of 
all noncommutative continuous functions on a quantum domain $\mathcal{E}=\left\{H_{\alpha}\right\}$ with its union space $\mathcal{D}=\bigcup_{\alpha} H_{\alpha}$.

Finally, confirm that some of the results of the present paper have been announced in 8 .

\section{Preliminaries}

The set of all positive integers is denoted by $\mathbb{N}$, and $\mathbb{C}$ denotes the complex number field. The unit circle in $\mathbb{C}$ centered at the origin is denoted by $\mathbb{T}$. The direct product of complex linear spaces $E$ and $F$ is denoted by $E \times F$, and we put $E^{k}$ for the $k$-times product $E \times \cdots \times E$. The linear space of all linear transformations between $E$ and $F$ is denoted by $L(E, F)$, and we write $L(E)$ instead of $L(E, E)$. The identity operator on $E$ is denoted by $I_{E}$ or just $I$. It is the unit of the associative algebra $L(E)$. Take $T \in L(E)$. The $n$-fold inflation $T^{\oplus n}=T \oplus \cdots \oplus T \in L\left(E^{n}\right)$ of $T$ is acting as $\left(x_{i}\right)_{i} \mapsto\left(T x_{i}\right)_{i}, x_{i} \in E, 1 \leq i \leq n$. If $T$ leaves invariant a subspace $F \subseteq E$, then $T \mid F$ denotes the restriction of $T$ onto $F$. The kernel of $T$ is denoted by $\mathcal{N}(T)$.

The domain of an unbounded operator $T$ on a Hilbert space $H$ is denoted by $\mathcal{D}(T)$. The range of $T$ is denoted by $\mathcal{R}(T)$. For unbounded operators $T$ and $S$ on $H$ we write $T \subseteq S$ if $\mathcal{D}(T) \subseteq \mathcal{D}(S)$ and $T x=S x$ for all $x \in \mathcal{D}(T)$. If $T$ is a densely defined operator on $H$, then $T^{\star}$ denotes its dual operator. Thus $\langle T x, y\rangle=\left\langle x, T^{\star} y\right\rangle$ for all $x \in \mathcal{D}(T), y \in \mathcal{D}\left(T^{\star}\right)$, where $\langle\cdot, \cdot\rangle$ is the inner product in $H$. An unbounded operator $T$ on $H$ is said to be symmetric if $T \subseteq T^{\star}$ (see [20, 13.13.3]); that is, $\langle T x, y\rangle=\langle x, T y\rangle$ whenever $x, y \in \mathcal{D}(T)$. If $T=T^{\star}$, then $T$ is said to be self-adjoint. The closure of a closable operator $T$ is denoted by $\bar{T}$. Recall that a densely defined closed operator $T$ on a Hilbert space $H$ is said to be normal if $\mathcal{D}(T)=\mathcal{D}\left(T^{\star}\right)$ and $T^{\star} T=T T^{\star}[20,13.13 .29]$. Obviously, every self-adjoint operator is normal.

The $C^{*}$-algebra of all continuous functions $f: \Omega \rightarrow \mathbb{C}$ on a compact (Hausdorff) space $\Omega$ with the uniform norm $\|f\|_{\infty}=\sup \{|f(\omega)|: \omega \in \Omega\}$ is denoted by $C(\Omega)$. The $C^{*}$-algebra of all bounded linear operators on a Hilbert space $H$ is denoted by $\mathcal{B}(H)$. The usual spectrum of an operator $T \in \mathcal{B}(H)$ is denoted by $\sigma(T)$. A unital commutative $C^{*}$-subalgebra $A \subseteq \mathcal{B}(H)$ is called a normal algebra. A typical example of a normal algebra is the unital $C^{*}$-algebra generated by a normal operator. The space of characters of $A$ equipped with the weak* topology from the norm dual $A^{*}$ is denoted by $\operatorname{Spec}(A)$. The Gelfand transform of an element $T \in A$ is denoted by $\widehat{T}$, so, $\widehat{T} \in C(\operatorname{Spec}(A)), \widehat{T}(\lambda)=\lambda(T)$.

Lemma 2.1. Let $A$ be a normal algebra in $\mathcal{B}(H)$ and let $M \subseteq H$ be a reducing subspace of $A$. Then $A \mid M=\{T \mid M: T \in A\}$ is a normal algebra in $\mathcal{B}(M)$, and if $E$ and $E^{\prime}$ are spectral measures of $A$ and $A \mid M$, respectively, then $E(\omega) \mid M=$ $E^{\prime}(\omega \cap \operatorname{Spec}(A \mid M))$ for each Borel subset $\omega \subseteq \operatorname{Spec}(A)$.

Proof. First, note the restriction mapping $A \rightarrow \mathcal{B}(M), T \mapsto T \mid M$, is a $*$-homomorphism, since $M$ is a reducing subspace of $A$. It follows that $A \mid M$ as the range of this $*$-homomorphism is a commutative $C^{*}$-algebra (see for instance [15, 4.7.83]). If $I_{M}=\{T \in A: T \mid M=0\}$ is the kernel of the $*$-homomorphism, then the canonical identification $(A \mid M)^{*} \simeq I_{M}^{\perp}$ is a weak* homeomorphism, where $I_{M}^{\perp} \subseteq A^{*}$ is the annihilator of $I_{M}$. In particular, $\operatorname{Spec}(A \mid M)$ is identified with a compact subset 
in $\operatorname{Spec}(A)$. Fix $x, y \in M$. If $T \in A$, then $\left.\widehat{T}\right|_{\operatorname{Spec}(A \mid M)}(\lambda)=\lambda(T)=\lambda(T \mid M)=$ $\widehat{T \mid M}(\lambda)$ and

$$
\begin{aligned}
\int_{\operatorname{Spec}(A)} \widehat{T} d E_{x, y} & =\langle T x, y\rangle=\langle T \mid M x, y\rangle \\
& =\int_{\operatorname{Spec}(A \mid M)} \widehat{T \mid M} d E_{x, y}^{\prime}=\int_{\operatorname{Spec}(A)} \widehat{T} \chi_{\operatorname{Spec}(A \mid M)} d E_{x, y}^{\prime} .
\end{aligned}
$$

It follows that $d E_{x, y}=\chi_{\operatorname{Spec}(A \mid M)} d E_{x, y}^{\prime}$ or $E_{x, y}(\omega)=E_{x, y}^{\prime}(\omega \cap \operatorname{Spec}(A \mid M))$ for each Borel subset $\omega \subseteq \operatorname{Spec}(A)$. But the latter is true for all $x, y \in M$, hence $E(\omega) \mid M=E^{\prime}(\omega \cap \operatorname{Spec}(A \mid M))$.

The following lemma of the mathematical folklore will be used later, and its short proof has been proposed by F.-H. Vasilescu.

Lemma 2.2. Let $A$ be a normal algebra in $\mathcal{B}(H)$ and let $\left\{H_{\alpha}\right\}_{\alpha \in \Lambda}$ be an upward filtered family reducing subspaces of $A$ such that their union $\bigcup_{\alpha \in \Lambda} H_{\alpha}$ is dense in $H$. Then $\operatorname{Spec}(A)$ is the closure of the union of all $\operatorname{Spec}\left(A \mid H_{\alpha}\right)$; that is,

$$
\operatorname{Spec}(A)=\overline{\bigcup_{\alpha \in \Lambda} \operatorname{Spec}\left(A \mid H_{\alpha}\right)} \text {. }
$$

Proof. Each $A \mid H_{\alpha}$ is a normal algebra in $\mathcal{B}\left(H_{\alpha}\right)$ thanks to Lemma 2.1. Let $E_{\alpha}$ be the spectral measure of $A \mid H_{\alpha}, E$ the spectral measure of $A, \sigma=\operatorname{Spec}(A)$, and let $\sigma_{\alpha}=\operatorname{Spec}\left(A \mid H_{\alpha}\right)$ for all $\alpha \in \Lambda$. We also set $\sigma_{0}=\overline{\bigcup_{\alpha \in \Lambda} \sigma_{\alpha}}$. Clearly, $\sigma_{0} \subseteq \sigma$. By Lemma 2.1, $E(\omega) \mid H_{\alpha}=E_{\alpha}\left(\omega \cap \sigma_{\alpha}\right)$ for each Borel set $\omega \subseteq \sigma$. If $\sigma_{0} \neq \sigma$, then $\sigma \backslash \sigma_{0}$ is a nonempty open subset in $\sigma$. Therefore $E\left(\sigma \backslash \sigma_{0}\right) \neq 0[20,12.22$ (d)]. Take a unit vector $x \in \mathcal{R}\left(E\left(\sigma \backslash \sigma_{0}\right)\right)$. Taking into account that the union $\bigcup H_{\alpha}$ is dense in $H$, we conclude that there exists a sequence $\left(x_{\alpha_{n}}\right)$ convergent to $x$, where $x_{\alpha_{n}} \in H_{\alpha_{n}}, n \in \mathbb{N}$. Using Lemma 2.1 again, infer that $E\left(\sigma_{0}\right) x=$ $\lim _{n} E\left(\sigma_{0}\right) x_{\alpha_{n}}=\lim _{n}\left(E\left(\sigma_{0}\right) \mid H_{\alpha_{n}}\right) x_{\alpha_{n}}=\lim _{n}\left(E_{\alpha_{n}}\left(\sigma_{\alpha_{n}}\right)\right) x_{\alpha_{n}}=\lim _{n} x_{\alpha_{n}}=x ;$ that is, $x \in \mathcal{R}\left(E\left(\sigma_{0}\right)\right)$. But the ranges of the projections $E\left(\sigma \backslash \sigma_{0}\right)$ and $E\left(\sigma_{0}\right)$ are orthogonal to each other. Then $x=0$, a contradiction. Whence $\sigma_{0}=\sigma$.

Corollary 2.1. Let $T \in \mathcal{B}(H)$ be a normal operator and let $\left\{H_{\alpha}\right\}_{\alpha \in \Lambda}$ be an upward filtered family reducing subspaces of $T$ such that their union $\bigcup_{\alpha \in \Lambda} H_{\alpha}$ is dense in $H$. Then $\sigma(T)=\overline{\bigcup_{\alpha \in \Lambda} \sigma\left(T \mid H_{\alpha}\right)}$.

Proof. It suffices to apply Lemma 2.2 to the normal algebra $A$ generated by $T$ and to use the well known formula $\sigma(T)=\{\widehat{T}(\lambda): \lambda \in \operatorname{Spec}(A)\}$.

2.1. Quantum spaces. The linear space of all $m \times n$-matrices $x=\left[x_{i j}\right]$ over a linear space $E$ is denoted by $\mathbb{M}_{m, n}(E)$, and we set $\mathbb{M}_{m}(E)=\mathbb{M}_{m, m}(E), \mathbb{M}_{m, n}=$ $\mathbb{M}_{m, n}(\mathbb{C})$. Further, $\mathbb{M}(E)$ denotes the linear space of all infinite matrices $\left[x_{i j}\right]$ $\left(x_{i j} \in E\right)$, where all but finitely many of $x_{i j}$ are zero. If $E=\mathbb{C}$ we write $\mathbb{M}$ instead of $\mathbb{M}(\mathbb{C})$. Each $\mathbb{M}_{m, n}(E)$ is a subspace in $\mathbb{M}(E)$ comprising those matrices $x=\left[x_{i j}\right]$ with $x_{i j}=0$ whenever $i>m$ or $j>n$. Moreover, $\mathbb{M}(E)=\underline{\lim } \mathbb{M}_{m, n}(E)$ is the inductive limit of these subspaces. Note that $\mathbb{M}_{m, n}(L(E))=L\left(E^{n}, E^{m}\right)$ 
up to a canonical identification. In particular, $\mathbb{M}_{n}(L(E))=L\left(E^{n}\right)$. The space $\mathbb{M}_{m, n}(E)$ (respectively, $\mathbb{M}(E)$ ) equipped with a certain polynormed (or locally convex) topology, is denoted by $M_{m, n}(E)$ (respectively, $M(E)$ ). For instance, if $E=H$ is a Hilbert space, then $\mathbb{M}_{n}(\mathcal{B}(H))=\mathcal{B}\left(H^{n}\right)=M_{n}(\mathcal{B}(H))$. In particular, $M_{m, n}$ is the space $\mathbb{M}_{m, n}$ with the operator norm $\|\cdot\|$ between the canonical Hilbert spaces $\mathbb{C}^{n}$ and $\mathbb{C}^{m}$. Take $a \in \mathbb{M}_{m, s}, v \in \mathbb{M}_{s, t}(E)$ and $b \in \mathbb{M}_{t, n}$. The matrix product $a v b \in \mathbb{M}_{m, n}(E)$ is defined by the usual way, $a v b=\left[\sum_{k, l} a_{i k} v_{k l} b_{l j}\right]$. The direct sum of matrices $v \in \mathbb{M}_{s, t}(E)$ and $w \in \mathbb{M}_{m, n}(E)$ is denoted by $v \oplus w \in \mathbb{M}_{s+m, t+n}(E)$. A linear mapping $\varphi: E \rightarrow F$ has the canonical linear extensions $\varphi^{(n)}: \mathbb{M}_{n}(E) \rightarrow$ $\mathbb{M}_{n}(F), \varphi^{(n)}\left(\left[x_{i j}\right]\right)=\left[\varphi\left(x_{i j}\right)\right], n \in \mathbb{N}$, over all matrix spaces. We also have a linear mapping $\varphi^{(\infty)}: \mathbb{M}(E) \rightarrow \mathbb{M}(F)$ such that $\varphi^{(\infty)} \mid \mathbb{M}_{n}(E)=\varphi^{(n)}, n \in \mathbb{N}$.

Let $p^{(n)}: \mathbb{M}_{n}(E) \rightarrow[0, \infty], n \in \mathbb{N}$, be gauges (respectively, seminorms) over all matrix spaces. The family $p=\left(p^{(n)}\right)_{n \in \mathbb{N}}$ is said to be a matrix gauge (respectively, a matrix seminorm) [13, [12, 26] on $E$ if $p$ possesses the following properties

$$
\begin{gathered}
\text { M1 } p^{(m+n)}(v \oplus w) \leq \max \left\{p^{(m)}(v), p^{(n)}(w)\right\}, \\
\text { M2 } p^{(n)}(a v b) \leq\|a\| p^{(m)}(v)\|b\|
\end{gathered}
$$

for all $v=\left[v_{i j}\right] \in \mathbb{M}_{m}(E), w=\left[w_{i j}\right] \in \mathbb{M}_{n}(E), a \in M_{n, m}, b \in M_{m, n}, n, m \in \mathbb{N}$. Note that M2 implies that

$$
p^{(1)}\left(v_{i j}\right)=p^{(1)}\left(\varepsilon_{i} v \varepsilon_{j}^{*}\right) \leq p^{(m)}(v)=p^{(m)}\left(\sum \varepsilon_{i}^{*} v_{i j} \varepsilon_{j}\right) \leq \sum p^{(1)}\left(v_{i j}\right)
$$

for any $v=\left[v_{i j}\right] \in \mathbb{M}_{m}(E)$, where $\varepsilon_{i}$ are the canonical row matrices. Let $p$ and $q$ be matrix gauges on $E$. We write $p \preceq q$ whenever $p^{(n)} \leq q^{(n)}$ for all $n \in \mathbb{N}$. It is a partial order structure over all matrix gauges on $E$. In particular, we define $\sup _{\iota} p_{\iota}=\left\{\sup _{\iota} p_{\iota}^{(n)}: n \in \mathbb{N}\right\}$ for a family $\left\{p_{\iota}\right\}$ of matrix gauges on $E$. Note that for a matrix gauge $p$ on $E$, M1 implies that we have a well defined gauge $p^{(\infty)}: \mathbb{M}(E) \rightarrow[0, \infty]$ given by the rule $p^{(\infty)}(x)=p^{(n)}(x), x \in \mathbb{M}_{n}(E)$. Furthermore the relation $p \preceq q$ for matrix gauges turns out to be a usual relation $p^{(\infty)} \leq q^{(\infty)}$ between the gauges on $\mathbb{M}(E)$. If $p$ is a matrix gauge, then the corresponding $p^{(\infty)}$ is a $\mathbb{M}$-module gauge on $\mathbb{M}(E)$ 13; that is, $p^{(\infty)}(x+y)=$ $\max \left\{p^{(\infty)}(x), p^{(\infty)}(y)\right\}$ for orthogonal elements $x, y \in \mathbb{M}(E)$, and $p^{(\infty)}(a x b) \leq$ $\|a\| p^{(\infty)}(x)\|b\|$ for all $a, b \in \mathbb{M}, x \in \mathbb{M}(E)$. Moreover, this correspondence is a one-to-one relation between the matrix gauges on $E$ and $\mathbb{M}$-module gauges on $\mathbb{M}(E)$ [13. If $\left\{p_{\iota}\right\}$ is a family of matrix seminorms on $E$, then obviously $\sup _{\iota} p_{\iota}$ is a matrix gauge on $E$ and $\left(\sup _{\iota} p_{\iota}\right)^{(\infty)}=\sup _{\iota} p_{\iota}^{(\infty)}$.

A linear space $E$ with a (separated) family of matrix seminorms $\left\{p_{\alpha}: \alpha \in \Lambda\right\}$ is called an abstract quantum space or local operator space. Note that the quantum space structure on $E$ determines a polynormed (Hausdorff) topology on $\mathbb{M}(E)$ by means of the family of seminorms $\left\{p_{\alpha}^{(\infty)}: \alpha \in \Lambda\right\}$. The relevant polynormed space is denoted by $M(E)$. A linear space $E$ is said to be a normed quantum space or an operator space if $E$ is equipped with a matrix norm. Recall that a concrete normed quantum space $E$ is defined as a subspace of $\mathcal{B}(H)$ for a certain Hilbert space $H$ (see [12, 2.1]). The inclusions $\mathbb{M}_{n}(E) \subseteq M_{n}(\mathcal{B}(H))=\mathcal{B}\left(H^{n}\right), n \in \mathbb{N}$, determine the relevant matrix norm on $E$. 
Let $E$ be a quantum space. Then each matrix space $\mathbb{M}_{n}(E)$ turns into a polynormed space (or a normed space in the normed quantum space case) denoted by $M_{n}(E)$ with a defining family of seminorms $\left\{p_{\alpha}^{(n)}: \alpha \in \Lambda\right\}$; that is, $M_{n}(E)$ is just a closed subspace in $M(E)$ (see (2.1)). The matrix seminorms $\left\{p_{\alpha}: \alpha \in \Lambda\right\}$ and $\left\{q_{\iota}: \iota \in \Omega\right\}$ on the same space $E$ are assumed to be equivalent if for each $\alpha \in \Lambda$ there corresponds a finite subset $F \subseteq \Omega$ and a positive constant $C_{\alpha F}$ such that $p_{\alpha} \preceq C_{\alpha F} \sup \left\{q_{\iota}: \iota \in F\right\}$ and vice versa; that is, the family of seminorms $\left\{p_{\alpha}^{(\infty)}: \alpha \in \Lambda\right\}$ and $\left\{q_{\iota}^{(\infty)}: \iota \in \Omega\right\}$ on $M(E)$ are equivalent in a usual manner. By a defining matrix seminorm family we mean any matrix seminorm family that is equivalent to the original one. Obviously, all equivalent families of matrix seminorms define the same topology on $M(E)$, in particular, over all matrix spaces $M_{n}(E)$, which is just the direct-product topology inherited by means of the canonical identifications $\mathbb{M}_{n}(E) \cong E^{n^{2}}$ (see (2.1)), $n \in \mathbb{N}$. Given a defining family of matrix seminorms, one also has its saturation $\left\{\sup \left\{p_{\alpha}: \alpha \in F\right\}: F \subseteq \Lambda\right\}$, where $F$ runs over all finite subsets. Note that the saturation is an upward filtered family of matrix seminorms which is equivalent to the original family. Thereby, when convenient, one can assume that the considered family of matrix seminorms is saturated.

Let $E$ and $F$ be quantum spaces with their (saturated) family of matrix seminorms $\left\{p_{\alpha}: \alpha \in \Lambda\right\}$ and $\left\{q_{\iota}: \iota \in \Omega\right\}$, respectively. A linear mapping $\varphi: E \rightarrow F$ is said to be matrix continuous if for each $\iota \in \Omega$ there corresponds $\alpha \in \Lambda$ and a positive constant $C_{\iota \alpha}$ such that $q_{\iota}^{(\infty)} \varphi^{(\infty)} \leq C_{\iota \alpha} p_{\alpha}^{(\infty)}$. If $\varphi$ is invertible and $\varphi^{-1}$ is matrix continuous too, then we say that $\varphi$ is a topological matrix isomorphism. If $C_{\iota \alpha} \leq 1$ for all possible $\iota$ and $\alpha$, then $\varphi$ is called a local matrix contraction with respect to the families $\left\{p_{\alpha}: \alpha \in \Lambda\right\}$ and $\left\{q_{\iota}: \iota \in \Omega\right\}$. Finally, a matrix injective mapping $\varphi$ (all $\varphi^{(n)}$ are injective) is said to be a local matrix isometry if $\Omega=\Lambda$ and $q_{\alpha}^{(\infty)} \varphi^{(\infty)}=p_{\alpha}^{(\infty)}$ for all $\alpha$.

2.2. Quantum domains. Let $H$ be a Hilbert space. By a quantum, or quantized domain (or merely a domain) in $H$ we mean an upward filtered family $\mathcal{E}=\left\{H_{\alpha}\right\}_{\alpha \in \Lambda}$ of closed subspaces in $H$ whose union $\mathcal{D}=\bigcup \mathcal{E}$ is dense in $H$. Note that $\mathcal{D}$ is a linear subspace in $H$ called the union space of the quantum domain $\mathcal{E}$. If $\mathcal{E}=\left\{H_{\alpha}\right\}_{\alpha \in \Lambda}$ and $\mathcal{K}=\left\{K_{\iota}\right\}_{\iota \in \Omega}$ are domains in $H$, then we write $\mathcal{E} \subseteq \mathcal{K}$ whenever $\Lambda=\Omega$ and $H_{\alpha} \subseteq K_{\alpha}$ for all $\alpha \in \Lambda$. Thus $\mathcal{E}=\mathcal{K}$ if and only if $\mathcal{E} \subseteq \mathcal{K}$ and $\mathcal{K} \subseteq \mathcal{E}$. Further, the domains $\mathcal{E}=\left\{H_{\alpha}\right\}_{\alpha \in \Lambda}$ and $\mathcal{K}=\left\{K_{\iota}\right\}_{\iota \in \Omega}$ in $H$ are assumed to be equivalent $\mathcal{E} \sim \mathcal{K}$ if for each $\alpha \in \Lambda$ there corresponds $\iota \in \Omega$ with $H_{\alpha} \subseteq K_{\iota}$, and vice versa. We note that the equivalent domains have the same union space. In particular, the disjoint union $\mathcal{E} \vee \mathcal{K}=\left\{H_{\alpha}, K_{\iota}\right\}_{\alpha \in \Lambda, \iota \in \Omega}$ is a domain in $H$ with the same union space $\mathcal{D}$, whenever $\mathcal{E} \sim \mathcal{K}$. Each domain $\mathcal{E}=\left\{H_{\alpha}\right\}_{\alpha \in \Lambda}$ in $H$ automatically associates a projection net $\mathfrak{p}=\left\{P_{\alpha}\right\}_{\alpha \in \Lambda}$ in $\mathcal{B}(H)$ over all subspaces $H_{\alpha}, \alpha \in \Lambda$, such that $1_{H}=\lim \mathfrak{p}($ WOT) (see [9]).

Let us introduce the algebra of all noncommutative continuous functions on a quantum domain $\mathcal{E}=\left\{H_{\alpha}\right\}_{\alpha \in \Lambda}$ with its union space $\mathcal{D}$ as

$$
C_{\mathcal{E}}(\mathcal{D})=\left\{T \in L(\mathcal{D}): T P_{\alpha}=P_{\alpha} T P_{\alpha} \in \mathcal{B}(H), \quad \alpha \in \Lambda\right\},
$$

where $\mathfrak{p}=\left\{P_{\alpha}\right\}_{\alpha \in \Lambda}$ is the projection net associated with $\mathcal{E}$. Thus $T\left(H_{\alpha}\right) \subseteq H_{\alpha}$ and $T \mid H_{\alpha} \in \mathcal{B}\left(H_{\alpha}\right)$ whenever $T \in C_{\mathcal{E}}(\mathcal{D})$. Obviously, $C_{\mathcal{E}}(\mathcal{D})$ is a unital subalgebra in $L(\mathcal{D})$. 
We also introduce the *-algebra of all noncommutative continuous functions on a quantum domain $\mathcal{E}$ as the subalgebra

$$
C_{\mathcal{E}}^{*}(\mathcal{D})=\left\{T \in C_{\mathcal{E}}(\mathcal{D}): P_{\alpha} T \subseteq T P_{\alpha}, \quad \alpha \in \Lambda\right\}
$$

in $C_{\mathcal{E}}(\mathcal{D})$. Each unbounded operator $T \in C_{\mathcal{E}}^{*}(\mathcal{D})$ has an unbounded dual $T^{\star}$ on $H$ such that

$$
\mathcal{D} \subseteq \mathcal{D}\left(T^{\star}\right), \quad T^{\star}(\mathcal{D}) \subseteq \mathcal{D} \quad \text { and } \quad T^{*}=T^{\star} \mid \mathcal{D} \in C_{\mathcal{E}}^{*}(\mathcal{D})
$$

The correspondence $T \mapsto T^{*}$ is an involution on $C_{\mathcal{E}}^{*}(\mathcal{D})$, thereby $C_{\mathcal{E}}^{*}(\mathcal{D})$ is a unital *-algebra. Conversely, if an unbounded operator $T \in C_{\mathcal{E}}(\mathcal{D})$ admits an unbounded dual $T^{\star}$ such that $T^{*}=T^{\star} \mid \mathcal{D} \in C_{\mathcal{E}}(\mathcal{D})$, then $T \in C_{\mathcal{E}}^{*}(\mathcal{D})$. In particular, $C_{\mathcal{E}}^{*}(\mathcal{D})$ consists of closable unbounded operators (see [9, Proposition 3.1] for the details). Moreover, if $C_{\mathcal{E}}(\mathcal{D})_{\text {sym }}$ is the set of all symmetric unbounded operators from the algebra $C_{\mathcal{E}}(\mathcal{D})$, then

$$
C_{\mathcal{E}}(\mathcal{D})_{\text {sym }}=C_{\mathcal{E}}^{*}(\mathcal{D})_{h},
$$

where $C_{\mathcal{E}}^{*}(\mathcal{D})_{h}$ is the set of all hermitian (or self-adjoint) elements of the $*$-algebra $C_{\mathcal{E}}^{*}(\mathcal{D})$.

Any Hilbert space $H$ can be treated as a quantum domain $\mathcal{E}=\{H\}$. In this case $C_{\mathcal{E}}(\mathcal{D})=C_{\mathcal{E}}^{*}(\mathcal{D})=\mathcal{B}(H)$. Note also that the family of all finite dimensional subspaces of a dense subspace in a Hilbert space is an example of a quantum domain. The algebra $C_{\mathcal{E}}^{*}(\mathcal{D})$ can be treated as a quantized version of the (commutative) multinormed $C^{*}$-algebra $C\left(\mathbb{R}^{n}\right)$ of all complex continuous functions on $\mathbb{R}^{n}$ equipped with the compact-open topology. Respectively, the space $\mathcal{D}$ can be referred to as a quantized version of the space $\mathbb{R}^{n}$ exhausted by an increasing family of its compact subsets. In this manner, one can also introduce the following algebra:

$$
C_{\mathcal{E}}^{*}(H)=\left\{T \in C_{\mathcal{E}}^{*}(\mathcal{D}): \mathcal{D}(T)=H\right\} .
$$

The elements of $C_{\mathcal{E}}^{*}(H)$ represent analogs of bounded continuous functions in $C\left(\mathbb{R}^{n}\right)$ or continuous functions over the point compactification of $\mathbb{R}^{n}$. The following assertion justifies this analog between classical and quantized theories.

Lemma 2.3. The algebra $C_{\mathcal{E}}^{*}(H)$ consists of bounded operators and

$$
C_{\mathcal{E}}^{*}(H)=\left\{T \in \mathcal{B}(H): T P_{\alpha}=P_{\alpha} T, \quad \alpha \in \Lambda\right\} .
$$

In particular, $C_{\mathcal{E}}^{*}(H)$ is a $C^{*}$-algebra in $\mathcal{B}(H)$.

Proof. Take $T \in C_{\mathcal{E}}^{*}(H)$. Then $T$ is an unbounded operator with $\mathcal{D}(T)=H$ and $T P_{\alpha}=P_{\alpha} T \in \mathcal{B}(H)$ for all $\alpha \in \Lambda$. It follows that $T x=\lim _{\alpha} P_{\alpha} T x$ for each $x \in H$. Taking into account that $\Lambda$ is an upward filtered set, we deduce that $\sup \left\{\left\|P_{\alpha} T x\right\|\right\}=\lim \left\|P_{\alpha} T x\right\|=\|T x\|<\infty$ for all $x \in H$. Using the Uniform Boundedness Principle (see for example [17, 7.2.5]), we deduce that $\sup \left\{\left\|P_{\alpha} T\right\|\right\}<$ $\infty$, which in turn implies that $\|T x\|=\sup \left\{\left\|P_{\alpha} T x\right\|\right\} \leq \sup \left\{\left\|P_{\alpha} T\right\|\right\}\|x\|$; that is, $T \in \mathcal{B}(H)$.

Now fix a positive integer $n$ and consider the $n$-th Hilbert space power $H^{n}$ of the Hilbert space $H$. If $\mathcal{E}=\left\{H_{\alpha}\right\}_{\alpha \in \Lambda}$ is a quantum domain in $H$ with its union space $\mathcal{D}$, then so is $\mathcal{E}^{n}=\left\{H_{\alpha}^{n}\right\}_{\alpha \in \Lambda}$ in $H^{n}$ whose union space is $\mathcal{D}^{n}$. If $\mathfrak{p}=\left\{P_{\alpha}\right\}_{\alpha \in \Lambda}$ is a projection net associated with $\mathcal{E}$, then so is $\mathfrak{p}^{\oplus n}=\left\{P_{\alpha}^{\oplus n}\right\}$ associated with $\mathcal{E}^{n}$. Furthermore, we have $\mathbb{M}_{n}\left(C_{\mathcal{E}}(\mathcal{D})\right)=C_{\mathcal{E}^{n}}\left(\mathcal{D}^{n}\right)$ and $\mathbb{M}_{n}\left(C_{\mathcal{E}}^{*}(\mathcal{D})\right)=$ $C_{\mathcal{E}^{n}}^{*}\left(\mathcal{D}^{n}\right)$. Thus if $T \in \mathbb{M}_{n}\left(C_{\mathcal{E}}(\mathcal{D})\right)$, then $T$ leaves invariant each subspace $H_{\alpha}^{n}$ and 
$\left\|T \mid H_{\alpha}^{n}\right\|_{\mathcal{B}\left(H_{\alpha}^{n}\right)}=\left\|P_{\alpha}^{\oplus n} T P_{\alpha}^{\oplus n}\right\|$. Put $p_{\alpha}=\left(p_{\alpha}^{(n)}\right)_{n \in \mathbb{N}}$, where $p_{\alpha}^{(n)}(T)=\left\|P_{\alpha}^{\oplus n} T P_{\alpha}^{\oplus n}\right\|$, $T \in \mathbb{M}_{n}\left(C_{\mathcal{E}}(\mathcal{D})\right), \alpha \in \Lambda$. The family $\left\{p_{\alpha}: \alpha \in \Lambda\right\}$ is an upward filtered family of matrix seminorms on $C_{\mathcal{E}}(\mathcal{D})$, which defines a matrix topology on $C_{\mathcal{E}}(\mathcal{D})$. If $\mathcal{E} \sim \mathcal{K}$ for some domain $\mathcal{K}$ in $H$, then both matrix topologies on $C_{\mathcal{E}}(\mathcal{D})$ and $C_{\mathcal{K}}(\mathcal{D})$ coincide on $C_{\mathcal{E}}(\mathcal{D}) \cap C_{\mathcal{K}}(\mathcal{D})=C_{\mathcal{E} \vee \mathcal{K}}(\mathcal{D})$. Moreover, $\left\{p_{\alpha}^{(1)}: \alpha \in \Lambda\right\}$ are multiplicative seminorms on $C_{\mathcal{E}}(\mathcal{D})$, which are $C^{*}$-seminorms on the $*$-subalgebra $C_{\mathcal{E}}^{*}(\mathcal{D})$. Thus $C_{\mathcal{E}}(\mathcal{D})$ has a canonical quantum space structure given by the family $\left\{p_{\alpha}: \alpha \in \Lambda\right\}$ of matrix seminorms associated with the domain $\mathcal{E}$. Moreover, the quantum space $C_{\mathcal{E}}(\mathcal{D})$ is complete; therefore $C_{\mathcal{E}}(\mathcal{D})$ is an Arens-Michael algebra [15, 2.1] whose *-subalgebra $C_{\mathcal{E}}^{*}(\mathcal{D})$ is a multinormed $C^{*}$-algebra 9 .

2.3. Concrete models. Now we introduce concrete quantum spaces, quantum systems and quantum $*$-algebras as relevant subspaces in $C_{\mathcal{E}}(\mathcal{D})$ compatible with its interior structures.

If $V$ is a linear subspace in $C_{\mathcal{E}}^{*}(\mathcal{D})$, then we set $V^{*}=\left\{T^{*}: T \in V\right\}$ for the space of all dual operators taken from $V$. A linear subspace $V \subseteq C_{\mathcal{E}}^{*}(\mathcal{D})$ is said to be self-adjoint (respectively, unital) if $V^{*}=V$ (respectively, $I_{\mathcal{D}} \in V$ ).

Definition 2.1. Any linear subspace in $C_{\mathcal{E}}(\mathcal{D})$ is called a concrete quantum space on a quantum domain $\mathcal{E}$. A unital self-adjoint subspace in $C_{\mathcal{E}}^{*}(\mathcal{D})$ is called a quantum system. If $T S \in V$ for all elements $T, S$ of a quantum system $V$, then we say that $V$ is a local operator algebra or a quantum $*$-algebra.

The completion of the concrete models introduced in Definition 2.1 remain the same concrete models. In particular, if $V \subseteq C_{\mathcal{E}}^{*}(\mathcal{D})$ is a local operator algebra, then its completion $\widetilde{V} \subseteq C_{\mathcal{E}}^{*}(\mathcal{D})$ is a multinormed $C^{*}$-algebra called a quantum $C^{*}$-algebra.

Let $V \subseteq C_{\mathcal{E}}^{*}(\mathcal{D})$ be a local operator algebra. An element $T \in V$ is called locally hermitian if $T=T^{*}$ on a certain subspace $H_{\alpha}$; that is, $T\left|H_{\alpha}=T^{*}\right| H_{\alpha}=\left(T \mid H_{\alpha}\right)^{*}$ in $\mathcal{B}\left(H_{\alpha}\right)$. In this case, we write $T={ }_{\alpha} T^{*}$. If the latter is true for all $\alpha$, we say that $T$ is (globally) hermitian; that is, $T \in C_{\mathcal{E}}^{*}(\mathcal{D})_{h}$. An element $T \in V$ is said to be locally positive if $T \geq_{\alpha} 0$; that is, $T \mid H_{\alpha} \geq 0$ in $\mathcal{B}\left(H_{\alpha}\right)$ for some $\alpha \in \Lambda$. Similarly, it is defined a (globally) positive element in $V$.

Let $V \subseteq C_{\mathcal{E}}^{*}(\mathcal{D})$ and $W \subseteq C_{\mathcal{K}}^{*}(\mathcal{O})$ be quantum systems on the quantum domains $\mathcal{E}=\left\{H_{\alpha}\right\}_{\alpha \in \Lambda}$ and $\mathcal{K}=\left\{K_{\iota}\right\}_{\iota \in \Omega}$ with their union spaces $\mathcal{D}$ and $\mathcal{O}$, respectively. A linear mapping $\varphi: V \rightarrow W$ is said to be locally positive if for each $\iota \in \Omega$ there corresponds $\alpha \in \Lambda$ such that $\varphi(v) \geq_{\iota} 0$ whenever $v \geq_{\alpha} 0$, and $\varphi(v)=_{\iota} 0$ if $v={ }_{\alpha} 0, v \in V$. For brevity, we write $\varphi(v)>_{\iota} 0$ whenever $v>_{\alpha} 0$. Further, a linear mapping $\varphi: V \rightarrow W$ is called local matrix positive if for each $\iota \in \Omega$ there corresponds $\alpha \in \Lambda$ such that $\varphi^{(n)}(v) \geq_{\iota} 0$ (that is, $\varphi^{(n)}(v) \mid K_{\iota}^{n} \geq 0$ ) whenever $v \geq_{\alpha} 0$ (that is, $v \mid H_{\alpha}^{n} \geq 0$ ), and $\varphi^{(n)}(v)={ }_{\iota} 0$ if $v={ }_{\alpha} 0, v \in M_{n}(V), n \in \mathbb{N}$. Thus $\varphi^{(n)}(v)>_{\iota} 0$ whenever $v>_{\alpha} 0, v \in M_{n}(V), n \in \mathbb{N}$. In particular, all $\varphi^{(n)}$ are local positive maps.

Finally, if for each $\iota \in \Omega$ there corresponds $\alpha \in \Lambda$ such that $\left\|\varphi^{(n)}(v)\right\|_{\mathcal{B}\left(K_{\iota}^{n}\right)} \leq$ $\|v\|_{\mathcal{B}\left(H_{\alpha}^{n}\right)}$ for all $v \in M_{n}(V), n \in \mathbb{N}$, then we say that $\varphi: V \rightarrow W$ is a local matrix contraction. Moreover, if $\Omega=\Lambda$ and $\left\|\varphi^{(n)}(v)\right\|_{\mathcal{B}\left(K_{\alpha}^{n}\right)}=\|v\|_{\mathcal{B}\left(H_{\alpha}^{n}\right)}$ for all $v \in M_{n}(V), n \in \mathbb{N}, \alpha \in \Lambda$, then $\varphi$ is called a local matrix isometry. 


\section{The Cayley transform}

In this section we use the Cayley transform to describe the multinormed $C^{*}$ algebra $C_{\mathcal{E}}^{*}(\mathcal{D})$ of all noncommutative continuous functions on a domain $\mathcal{E}$ with its union space $\mathcal{D}$.

3.1. The set $\mathfrak{U}_{\mathcal{E}}$ of unitary operators. Let $H$ be a Hilbert space and let $T$ be a densely defined operator on $H$. We investigate the Cayley transform of a symmetric operator $T$ which leaves invariant its domain $\mathcal{D}(T)$; that is, $T(\mathcal{D}(T)) \subseteq \mathcal{D}(T)$ (see also [21], 25]). For the sake of the reader we introduce some details of this construction.

Lemma 3.1. Let $T$ be a densely defined symmetric operator on $H$ such that $T(\mathcal{D}(T)) \subseteq \mathcal{D}(T)$. Then $T$ is self-adjoint iff $\mathcal{D}(T)=H$.

Proof. If $\mathcal{D}(T)=H$, then $T=T^{*}$, for $T$ is symmetric.

Now assume that $T$ is self-adjoint. So, $T$ is a densely defined closed operator. Then $\mathcal{R}\left(1+T^{2}\right)=H$ by virtue of $[20,13.13 .13]$. Since $(T+i)(T-i)=1+T^{2}$, it follows that $\mathcal{R}(T+i)=H$. But $(T+i) x=T x+i x \in \mathcal{D}(T)$ for $T(\mathcal{D}(T)) \subseteq \mathcal{D}(T)$; that is, $\mathcal{R}(T+i) \subseteq \mathcal{D}(T)$. Whence $\mathcal{D}(T)=H$.

Let $T$ be a densely defined symmetric operator such that $T(\mathcal{D}(T)) \subseteq \mathcal{D}(T)$ and let $U_{T}$ be the Cayley transform of $T$. According to the definition [20, 13.17], $U_{T}$ is an isometry between $\mathcal{D}\left(U_{T}\right)=\mathcal{R}(T+i)$ and $\mathcal{R}\left(U_{T}\right)=\mathcal{R}(T-i)$ such that

$$
U_{T}(T x+i x)=T x-i x, \quad x \in \mathcal{D}(T) .
$$

Note that $\mathcal{R}(T+i) \subseteq \mathcal{D}(T)$ and $\mathcal{R}(T-i) \subseteq \mathcal{D}(T)$. Moreover,

$$
I-U_{T}: \mathcal{D}\left(U_{T}\right) \longrightarrow \mathcal{R}\left(I-U_{T}\right)=\mathcal{D}(T)
$$

is one-to-one, and $I-U_{T}=2 i(T+i)^{-1}$.

Conversely, if $V$ is a partial isometry on $H$ such that $I-V: \mathcal{D}(V) \rightarrow \mathcal{R}(I-V)$ is one-to-one, then $V$ is the Cayley transform of a symmetric operator

$$
S: \mathcal{D}(S)=\mathcal{R}(I-V) \rightarrow \mathcal{R}(S), \quad S(z-V z)=i(z+V z), \quad z \in \mathcal{D}(V) .
$$

Thus $V=U_{S}$ and $\mathcal{D}(V)=\mathcal{R}(S+i), \mathcal{R}(V)=\mathcal{R}(S-i)$ [20, 13.13.19].

Lemma 3.2. Let $T$ be a densely defined symmetric operator on $H$. Then

$$
T(\mathcal{D}(T)) \subseteq \mathcal{D}(T) \quad \text { iff } \quad \mathcal{R}\left(I+U_{T}\right) \subseteq \mathcal{R}\left(I-U_{T}\right)
$$

Proof. Put $V=U_{T}$. Since $I+V=2 T(T+i)^{-1}$, it follows that $\mathcal{R}(I+V)=\mathcal{R}(T)$. Hence $T$ leaves invariant its domain if and only if $\mathcal{R}(I+V)=\mathcal{R}(T) \subseteq \mathcal{D}(T)=$ $\mathcal{R}(I-V)$; that is, $\mathcal{R}(I+V) \subseteq \mathcal{R}(I-V)$.

Now let $\mathcal{E}=\left\{H_{\alpha}: \alpha \in \Lambda\right\}$ be a quantum domain in $H$ with its union space $\mathcal{D}$ and let $T \in C_{\mathcal{E}}(\mathcal{D})_{\text {sym }}$. Then $T$ is a densely defined symmetric operator on $H$ such that $\mathcal{D}(T)=\mathcal{D}=\bigcup \mathcal{E}$. Moreover, $T \in C_{\mathcal{E}}^{*}(\mathcal{D})_{h}$, thanks to (2.4). Note that $(T \pm i)(\mathcal{E}) \subseteq \mathcal{E}$; that is, $(T \pm i)\left(H_{\alpha}\right) \subseteq H_{\alpha}$ for all $\alpha$. Furthermore, $T \pm i$ are $\mathcal{E}$ bijections; that is, $(T \pm i)\left|H_{\alpha}=T\right| H_{\alpha} \pm i$ are invertible in $\mathcal{B}\left(H_{\alpha}\right)$, respectively. So,

$$
\mathcal{R}(T \pm i)=\bigcup_{\alpha \in \Lambda} \mathcal{R}\left((T \pm i) \mid H_{\alpha}\right)=\bigcup_{\alpha \in \Lambda} H_{\alpha}=\mathcal{D} .
$$

Therefore, $T \pm i: \mathcal{D} \rightarrow \mathcal{D}$ are bijections such that $(T \pm i)^{-1} \mid H_{\alpha}=\left(T \mid H_{\alpha} \pm i\right)^{-1} \in$ $\mathcal{B}\left(H_{\alpha}\right)$ for all $\alpha$. It follows that $\mathcal{D}\left(U_{T}\right)=\mathcal{R}(T+i)=\mathcal{D}$ and $\mathcal{R}\left(U_{T}\right)=\mathcal{R}(T-i)=$ 
$\mathcal{D}$; therefore $U_{T}: \mathcal{D} \rightarrow \mathcal{D}$ is an isometry of $\mathcal{D}$ onto $\mathcal{D}$. In particular, $U_{T}$ has a unique unitary extension $\bar{U}_{T} \in \mathcal{B}(H)$.

Lemma 3.3. Let $T \in C_{\mathcal{E}}(\mathcal{D})_{\text {sym }}$ and let $U_{\bar{T}}$ be the Cayley transform of the closure $\bar{T}$ of $T$. Then $U_{\bar{T}}=\bar{U}_{T}$ and $\bar{T}$ is a self-adjoint unbounded operator.

Proof. Since $T \subseteq T^{\star}$, the closure $\bar{T}$ is a closed symmetric operator on $H$. In particular, $\mathcal{R}(\bar{T} \pm i)$ are closed subspaces [20, 13.13.16]. But $\mathcal{D}=\mathcal{R}(T \pm i) \subseteq$ $\mathcal{R}(\bar{T} \pm i)$ for $T \pm i$ are $\mathcal{E}$-bijections. Then $\mathcal{R}(\bar{T} \pm i)=H$ or $\mathcal{D}\left(U_{\bar{T}}\right)=\mathcal{R}\left(U_{\bar{T}}\right)=H$. Hence $U_{\bar{T}}=\bar{U}_{T}$. In particular, $U_{\bar{T}}$ is unitary and $\bar{T}$ is a self-adjoint unbounded operator [20, 13.13.19].

$U_{T}$ being the Cayley transform of $T$, we conclude that

$$
\left(I-U_{\bar{T}}\right) \mid \mathcal{D}=I-U_{T}: \mathcal{D} \rightarrow \mathcal{D}
$$

is one-to-one and onto. In particular, $\mathcal{N}\left(I-U_{\bar{T}}\right)=\mathcal{N}\left(I-U_{\bar{T}}^{*}\right)=\mathcal{R}\left(I-U_{\bar{T}}\right)^{\perp} \subseteq$ $\mathcal{R}\left(I-U_{T}\right)^{\perp}=\mathcal{D}^{\perp}=\{0\}$; that is, 1 does not belong to the point spectrum of $U_{\bar{T}}$.

Proposition 3.1. If $T \in C_{\mathcal{E}}(\mathcal{D})_{\mathrm{sym}}$, then $\sigma\left(U_{\bar{T}}\right)=\overline{\bigcup_{\alpha \in \Lambda} \sigma\left(U_{T} \mid H_{\alpha}\right)}$ and $1 \notin$ $\bigcup_{\alpha \in \Lambda} \sigma\left(U_{\bar{T}} \mid H_{\alpha}\right)$. Moreover, $1 \in \sigma\left(U_{\bar{T}}\right)$ iff $T$ is discontinuous.

Proof. Since $U_{\bar{T}}$ is unitary (see Lemma 3.3) and $U_{\bar{T}}\left(H_{\alpha}\right)=H_{\alpha}$, we conclude that $U_{\bar{T}}^{*}\left(H_{\alpha}\right)=U_{\bar{T}}^{-1}\left(H_{\alpha}\right) \subseteq H_{\alpha}$, which in turn implies that $U_{\bar{T}} P_{\alpha}=P_{\alpha} U_{\bar{T}} P_{\alpha}=$ $\left(P_{\alpha} U_{\bar{T}}^{*} P_{\alpha}\right)^{*}=\left(U_{\bar{T}}^{*} P_{\alpha}\right)^{*}=P_{\alpha} U_{\bar{T}}$ for all $\alpha \in \Lambda$, where $P_{\alpha}$ is the projection onto $H_{\alpha}$. It remains to use Corollary 2.1

Further, $\left(I-U_{T}\right)\left(T x_{\alpha}+i x_{\alpha}\right)=2 i x_{\alpha}, x_{\alpha} \in H_{\alpha}$; therefore $\left(I-\bar{U}_{T}\right) H_{\alpha}=H_{\alpha}$ for all $\alpha$. Thus $U_{\bar{T}}\left|H_{\alpha}=U_{T}\right| H_{\alpha}$ is the Cayley transform of the selfadjoint and bounded operator $T \mid H_{\alpha}$. Hence $1 \notin \sigma\left(U_{\bar{T}} \mid H_{\alpha}\right)$ for each $\alpha$ (see Remark 3.1 below).

Finally, if $T$ is bounded, then $\bar{T}$ is a bounded self-adjoint operator whose Cayley transform $U_{\bar{T}}$ is unitary and $1 \notin \sigma\left(U_{\bar{T}}\right)$. Conversely, if $1 \notin \sigma\left(U_{\bar{T}}\right)$, then $\bar{T}=$ $i\left(I+U_{\bar{T}}\right)\left(I-U_{\bar{T}}\right)^{-1}$ is bounded. In particular, $T$ is continuous.

Remark 3.1. If $T$ is bounded, then $T$ has the self-adjoint extension $\bar{T} \in \mathcal{B}(H)$ (Lemma 3.1). So $\mathcal{R}(\bar{T}+i)=H$. Take $x \in H$ and let $x=\lim \left\{x_{n}\right\}, x_{n} \in \mathcal{D}$. Then $U_{\bar{T}}(\bar{T} x+i x)=\lim \bar{U}_{T}\left(T x_{n}+i x_{n}\right)=\lim U_{T}\left(T x_{n}+i x_{n}\right)=\lim T x_{n}-i x_{n}=$ $\bar{T} x-i x$. Thus $U_{\bar{T}}(\bar{T}+i)=\bar{T}-i$. Since $(\bar{T}+i)^{-1} \in \mathcal{B}(H)$, it follows that $U_{\bar{T}}=(\bar{T}-i)(\bar{T}+i)^{-1}=r(\bar{T})$, where $r(t)=(t-i)(t+i)^{-1}, t \in \mathbb{R}$, is a rational function. In particular, $\sigma\left(U_{\bar{T}}\right)=r(\sigma(\bar{T})) \subseteq \mathbb{T} \backslash\{1\}$.

Theorem 3.1. Let $\mathcal{E}=\left\{H_{\alpha}: \alpha \in \Lambda\right\}$ be a domain in a Hilbert space with its union space $\mathcal{D}$ and let $\mathfrak{U}_{\mathcal{E}} \subseteq \mathcal{B}(H)$ be a subset of all unitary operators $V$ such that $V\left(H_{\alpha}\right)=H_{\alpha}, \alpha \in \Lambda$, and $1 \notin \bigcup_{\alpha \in \Lambda} \sigma\left(V \mid H_{\alpha}\right)$. The Cayley transform $T \mapsto U_{\bar{T}}$ implements a bijection

$$
C_{\mathcal{E}}(\mathcal{D})_{\mathrm{sym}} \rightarrow \mathfrak{U}_{\mathcal{E}}
$$

Moreover, $T$ is bounded iff $1 \notin \sigma\left(U_{\bar{T}}\right)$. 
Proof. Take $V \in \mathfrak{U}_{\mathcal{E}}$. Then $V\left(H_{\alpha}\right)=H_{\alpha}$ and $1 \notin \sigma\left(V \mid H_{\alpha}\right)$ for each $\alpha$. Therefore $V(\mathcal{D})=\mathcal{D}$ and

$$
\mathcal{R}(I-V \mid \mathcal{D})=\bigcup_{\alpha} \mathcal{R}\left(I-V \mid H_{\alpha}\right)=\bigcup_{\alpha} H_{\alpha}=\mathcal{D}
$$

that is, $I-V \mid \mathcal{D}: \mathcal{D} \rightarrow \mathcal{D}$ is one-to-one and onto. In particular, $\mathcal{R}(I+V \mid \mathcal{D}) \subseteq$ $\mathcal{D}=\mathcal{R}(I-V \mid \mathcal{D})$. Put

$$
S: \mathcal{D}(S)=\mathcal{D} \rightarrow \mathcal{D}, \quad S(x-V x)=i(x+V x), \quad x \in \mathcal{D} .
$$

If $x \in H_{\alpha}$, then $S(x-V x) \in H_{\alpha}$; that is, $S\left(H_{\alpha}\right) \subseteq H_{\alpha}$ for all $\alpha$. Moreover, $V \mid \mathcal{D}=U_{S}$ thanks to [20, 13.13.19] and Lemma 3.2, Therefore $V=\bar{U}_{S}$ or $V=U_{\bar{S}}$ by virtue of Lemma 3.3. Taking into account that $S=i(1+V \mid \mathcal{D})(1-V \mid \mathcal{D})^{-1}$, we conclude that $S \mid H_{\alpha} \in \mathcal{B}\left(H_{\alpha}\right)$ for all $\alpha$. In particular, $S \in C_{\mathcal{E}}(\mathcal{D})_{\text {sym }}$.

Since the Cayley transform $T \mapsto U_{T}$ is one-to-one, it follows that the mapping $C_{\mathcal{E}}(\mathcal{D})_{\text {sym }} \rightarrow \mathfrak{U}_{\mathcal{E}}, T \mapsto U_{\bar{T}}$ is a bijection.

Finally, $T$ is bounded iff $1 \notin \sigma\left(U_{\bar{T}}\right)$, by virtue of Proposition 3.1

3.2. The relationship between spectra. Now we propose some relationships between the sets $\sigma\left(\bar{U}_{T}\right) \backslash \bigcup_{\alpha \in \Lambda} \sigma\left(U_{T} \mid H_{\alpha}\right)$ and $\overline{\mathbb{R} \backslash \sigma\left(T \mid H_{\beta}\right)}$. Let us introduce the function $f: \mathbb{T} \backslash\{1\} \rightarrow \mathbb{R}$ given by the rule

$$
f(\lambda)=\left\{\begin{array}{ccc}
\left(\frac{1+\operatorname{Re}(\lambda)}{1-\operatorname{Re}(\lambda)}\right)^{1 / 2}, & \text { if } & \operatorname{Im}(\lambda) \leq 0, \\
-\left(\frac{1+\operatorname{Re}(\lambda)}{1-\operatorname{Re}(\lambda)}\right)^{1 / 2}, & \text { if } & \operatorname{Im}(\lambda)>0 .
\end{array}\right.
$$

One can easily verify that $f$ is a continuous bijection such that $f\{\operatorname{Im}(\lambda) \leq 0\}=$ $\{r \geq 0\}$ and $f\{\operatorname{Im}(\lambda)>0\}=\{r<0\}$.

Proposition 3.2. If $T \in C_{\mathcal{E}}(\mathcal{D})_{\mathrm{sym}}$ is a discontinuous operator, then

$$
f\left(\sigma\left(\bar{U}_{T}\right) \backslash\{1\} \cup \bigcup_{\alpha \in \Lambda} \sigma\left(U_{T} \mid H_{\alpha}\right)\right) \subseteq \overline{\mathbb{R} \backslash \sigma\left(T \mid H_{\beta}\right)}
$$

for each $\beta \in \Lambda$.

Proof. Since $T$ is not bounded, we have $1 \in \sigma\left(\bar{U}_{T}\right)$ thanks to Proposition 3.1. Fix $\beta \in \Lambda$ and consider an upward filtered set $\Omega=\{\alpha \in \Lambda: \alpha \geq \beta\}$. Take $\lambda \in$ $\sigma\left(\bar{U}_{T}\right) \backslash \bigcup_{\alpha \in \Lambda} \sigma\left(U_{T} \mid H_{\alpha}\right)$ with $\lambda \neq 1$. Put $c_{\lambda}=2|\operatorname{Im}(\lambda)||\lambda-1|^{-2}$. If $\lambda=a+i b$, then

$$
\begin{aligned}
c_{\lambda} & =\frac{2|b|}{(a-1)^{2}+b^{2}}=\frac{2|b|}{a^{2}+1-2 a+b^{2}}=\frac{|b|}{1-a}=\frac{\sqrt{1-a^{2}}}{1-a} \\
& =\frac{\sqrt{1-a} \sqrt{1+a}}{1-a}=\frac{\sqrt{1+a}}{\sqrt{1-a}}=\left(\frac{1+\operatorname{Re}(\lambda)}{1-\operatorname{Re}(\lambda)}\right)^{1 / 2} ;
\end{aligned}
$$

recall that $\lambda \in \mathbb{T}$. Hence $f(\lambda)=c_{\lambda}$ if $\operatorname{Im}(\lambda) \leq 0$, and $f(\lambda)=-c_{\lambda}$ if $\operatorname{Im}(\lambda)>0$.

By assumption $\lambda \notin \bigcup_{\alpha \in \Lambda} \sigma\left(U_{T} \mid H_{\alpha}\right)$. There are positive constants $\delta_{\alpha}(\lambda)$ with $\left\|\left(\lambda-U_{T} \mid H_{\alpha}\right) z_{\alpha}\right\| \geq \delta_{\alpha}(\lambda)\left\|z_{\alpha}\right\|$ for all $z_{\alpha}=T x_{\alpha}+i x_{\alpha} \in H_{\alpha}, x_{\alpha} \in H_{\alpha}, \alpha \in \Omega$. One may assume that $\delta_{\alpha}(\lambda) \downarrow 0$. Indeed, if $\delta_{\alpha}(\lambda) \geq C(\lambda)>0$ for all $\alpha \in \Omega$, then $\left\|\left(\lambda-\bar{U}_{T}\right) z\right\| \geq C(\lambda)\|z\|$ for all $z \in \mathcal{D}$. $\mathcal{D}$ being a dense subspace in $H$, we 
conclude that the latter inequality is true for all $z \in H$. Since $\lambda-\bar{U}_{T}$ is normal, it follows that $\lambda \notin \sigma\left(\bar{U}_{T}\right)$ (see [20, 12.12.12]), a contradiction.

Finally, using similar arguments one can derive that $f(\lambda) \in \overline{\mathbb{R} \backslash \sigma\left(T \mid H_{\beta}\right)}$. We omit the details.

Remark 3.2. $f$ being a bijection, we conclude from Proposition 3.2 that if spectra $\sigma\left(T \mid H_{\alpha}\right)$ are concentrated to the positive side in $\mathbb{R}$ and "rich" enough (for instance, connected), then the points from $\sigma\left(\bar{U}_{T}\right) \backslash \bigcup_{\alpha \in \Lambda} \sigma\left(U_{T} \mid H_{\alpha}\right)$ are located in the upper semicircle, and vice versa (see Corollary 3.1 below). In particular, if each $\sigma\left(T \mid H_{\alpha}\right)$ is a closed connected interval and $\bigcup_{\alpha \in \Lambda} \sigma\left(T \mid H_{\alpha}\right)=\mathbb{R}$, then

$$
\sigma\left(\bar{U}_{T}\right)=\{1\} \cup \bigcup_{\alpha \in \Lambda} \sigma\left(U_{T} \mid H_{\alpha}\right) .
$$

3.3. The normal elements in $C_{\mathcal{E}}^{*}(\mathcal{D})$. We say that $T \in C_{\mathcal{E}}^{*}(\mathcal{D})$ is a normal element if $T T^{*}=T^{*} T$. One can easily verify that $T$ is a normal element in $C_{\mathcal{E}}^{*}(\mathcal{D})$ iff $T=A+i B$ and $A B=B A$ for the uniquely determined hermitian elements $A, B \in C_{\mathcal{E}}^{*}(\mathcal{D})_{h}$. In particular, all hermitian elements in $C_{\mathcal{E}}^{*}(\mathcal{D})$ are normal. As we have mentioned in Subsection 2.2, the multinormed $C^{*}$-algebra $C_{\mathcal{E}}^{*}(\mathcal{D})$ consists of closable unbounded operators, and the closure $\bar{T}$ of a hermitian element $T \in C_{\mathcal{E}}^{*}(\mathcal{D})_{h}$ is a self-adjoint unbounded operator on $H$ thanks to Lemma 3.3 .

Proposition 3.3. Let $T \in C_{\mathcal{E}}^{*}(\mathcal{D})$. Then $T$ is a normal element if and only if $\bar{T}$ is an unbounded normal operator on $H$. In this case $\bar{T}=\bar{A}+i \bar{B}$ whenever $T=A+i B$.

Proof. If the closure $\bar{T}$ is a normal operator on $H$, then $D(\bar{T})=D\left(T^{\star}\right)$ and $T^{\star} \bar{T}=\bar{T} T^{\star}$. It follows that $D \subseteq D\left(T^{\star} \bar{T}\right), D \subseteq D\left(\bar{T} T^{\star}\right)$ and $T^{*} T=T^{\star} \bar{T} \mid D=$ $\bar{T} T^{\star} \mid D=T T^{*}$; that is, $T$ is a normal element in $C_{\mathcal{E}}^{*}(\mathcal{D})$.

Conversely, assume that $T$ is a normal element. First, let us prove that $\overline{T^{*}}=T^{\star}$. Since $T^{*}=A-i B \subseteq T^{\star}$, it follows that $\overline{T^{*}} \subseteq T^{\star}$. Take a point $\left(x, T^{\star} x\right)$ from the graph of the operator $T^{\star}$ which is orthogonal to the graph of $T^{*}$. Then $x \in \mathcal{D}\left(T^{\star}\right)$ and

$\left\langle x, y+T T^{*} y\right\rangle=\langle x, y\rangle+\left\langle x, T T^{*} y\right\rangle=\langle x, y\rangle+\left\langle T^{\star} x, T^{*} y\right\rangle=\left\langle\left(x, T^{\star} x\right),\left(y, T^{*} y\right)\right\rangle=0$

for all $y \in \mathcal{D}$. But $\left(I+T T^{*}\right)(\mathcal{D})=\mathcal{D}$. Indeed, $I+T T^{*}$ is $\mathcal{E}$-bijection, since

$$
\left(I+T T^{*}\right)\left(H_{\alpha}\right)=\left(I_{H_{\alpha}}+\left(T \mid H_{\alpha}\right)\left(T \mid H_{\alpha}\right)^{*}\right)\left(H_{\alpha}\right)=H_{\alpha} \quad \text { for all } \quad \alpha \in \Lambda .
$$

In particular, $\left(I+T T^{*}\right)(\mathcal{D})=\bigcup_{\alpha}\left(I+T T^{*}\right)\left(H_{\alpha}\right)=\mathcal{D}$. Consequently, $x \perp \mathcal{D}$; that is, $x=0$, for $\mathcal{D}$ is dense in $H$. In particular, $\overline{T^{*}}=T^{\star}$.

Since $T^{*} T=T T^{*}$, it follows that $\|T x\|=\left\|T^{*} x\right\|$ for all $x \in \mathcal{D}$. Then $\mathcal{D}(\bar{T})=$ $\mathcal{D}\left(\overline{T^{*}}\right)$. Indeed, if $z \in \mathcal{D}(\bar{T})$, then $z=\lim x_{n}$ and $\bar{T} z=\lim T x_{n}$ for a certain sequence $\left(x_{n}\right)$ in $\mathcal{D}$. But $\left(T^{*} x_{n}\right)$ is a Cauchy sequence in $H$, for $\left\|T^{*}\left(x_{n}-x_{m}\right)\right\|=$ $\left\|T\left(x_{n}-x_{m}\right)\right\|$. Therefore $\overline{T^{*}} z=\lim T^{*} x_{n}$. This means that $z \in \mathcal{D}\left(\overline{T^{*}}\right)$. Hence $\mathcal{D}(\bar{T}) \subseteq \mathcal{D}\left(\overline{T^{*}}\right) \subseteq \mathcal{D}\left(\overline{T^{* *}}\right)=\mathcal{D}(\bar{T})$. In particular, $\mathcal{D}(\bar{T})=\mathcal{D}\left(\overline{T^{*}}\right)=\mathcal{D}\left(T^{\star}\right)=$ $\mathcal{D}\left(\bar{T}^{\star}\right)$; that is, $\mathcal{D}(\bar{T})=\mathcal{D}\left(\bar{T}^{\star}\right)$. 
Finally, if $z=\lim x_{n} \in \mathcal{D}(\bar{T})$ with $\left(x_{n}\right) \subseteq \mathcal{D}$ and $\bar{T} z=\lim T x_{n}$, then $\left(T^{*} x_{n}\right)$ converges and $\|\bar{T} z\|=\lim \left\|T x_{n}\right\|=\lim \left\|T^{*} x_{n}\right\|=\lim \left\|T^{\star} x_{n}\right\|=\left\|\bar{T}^{\star} z\right\|$. Therefore, $\|\bar{T} z\|=\left\|\bar{T}^{\star} z\right\|$ for all $z \in \mathcal{D}(\bar{T})=\mathcal{D}\left(\bar{T}^{\star}\right)$. It follows that $\bar{T}$ is a normal operator (see [20, Ch. 13, Exercise 16]).

It remains to prove that $\bar{T}=\bar{A}+i \bar{B}$ whenever $T=A+i B$ with $A B=$ $B A$. By Lemma [3.3, $\bar{A}$ and $\bar{B}$ are self-adjoint operators and we have bounded operators $(\bar{A}+i)^{-1}$ and $(\bar{B}+i)^{-1}$. Since $(\bar{A}+i)^{-1} \mid H_{\alpha}=\left(A \mid H_{\alpha}+i\right)^{-1}$ and $(\bar{B}+i)^{-1} \mid H_{\alpha}=\left(B \mid H_{\alpha}+i\right)^{-1}$, it follows that $(\bar{A}+i)^{-1}$ and $(\bar{B}+i)^{-1}$ commute on $\mathcal{D}$. Hence $(\bar{A}+i)^{-1}$ and $(\bar{B}+i)^{-1}$ are commuting operators on $H$. In particular, $\bar{A}+i \bar{B}$ is a normal operator (see [25, Section 4]). Since $T=A+i B \subseteq \bar{A}+i \bar{B}$, it follows that $\bar{T} \subseteq \bar{A}+i \bar{B}$. But $\bar{T}$ is normal; therefore it is maximally normal 20 , 13.13.32]. Whence $\bar{T}=\bar{A}+i \bar{B}$.

3.4. The local realization for an operator $T \in C_{\mathcal{E}}(\mathcal{D})_{\mathrm{sym}}$. Let $\mathcal{E}=\left\{H_{\alpha}: \alpha \in \Lambda\right\}$ be a domain in a Hilbert space $H$ with its union space $\mathcal{D}$. An operator $S \in \mathcal{B}(H)$ is said to be a $\mathcal{D}$-bijection if $S(\mathcal{D}) \subseteq \mathcal{D}$ and $S \mid \mathcal{D}: \mathcal{D} \rightarrow \mathcal{D}$ is a bijection. Respectively, we say that $S$ is an $\mathcal{E}$-bijection if $S(\mathcal{E}) \subseteq \mathcal{E}$ and all restrictions $S \mid H_{\alpha}: H_{\alpha} \rightarrow H_{\alpha}$ are bijections; that is, $S \mid H_{\alpha}$ are invertible in $\mathcal{B}\left(H_{\alpha}\right)$, respectively. As follows from Theorem 3.1. all unitary operators from the class $\mathfrak{U}_{\mathcal{E}}$ introduced in Theorem 3.1 are $\mathcal{E}$-bijections.

Now let $T \in C_{\mathcal{E}}(\mathcal{D})_{\text {sym }}$ and put

$$
D_{T}=2 I-U_{\bar{T}}-U_{\bar{T}}^{*} \in \mathcal{B}(H),
$$

which is a hermitian operator. Actually, $D_{T} \geq 0$ for

$$
\sigma\left(D_{T}\right)=\left\{2-\lambda-\bar{\lambda}: \lambda \in \sigma\left(U_{\bar{T}}\right)\right\}=\left\{2(1-\operatorname{Re}(\lambda)): \lambda \in \sigma\left(U_{\bar{T}}\right)\right\} \geq 0 .
$$

Moreover, $D_{T}$ leaves invariant each subspace $H_{\alpha}$ and $D_{T}\left|H_{\alpha}=2-U_{T}\right| H_{\alpha}-$ $\left(U_{T} \mid H_{\alpha}\right)^{*}$, which in turn implies that

$$
\sigma\left(D_{T} \mid H_{\alpha}\right)=\left\{2(1-\operatorname{Re}(\lambda)): \lambda \in \sigma\left(U_{T} \mid H_{\alpha}\right)\right\}>0
$$

for $1 \notin \sigma\left(U_{T} \mid H_{\alpha}\right)$. Thus $\left(D_{T} \mid H_{\alpha}\right)^{-1} \in \mathcal{B}\left(H_{\alpha}\right)$ for all $\alpha$. The latter means that $D_{T}$ is an $\mathcal{E}$-bijection. We also set

$$
W_{T}=i\left(U_{\bar{T}}-U_{\bar{T}}^{*}\right) \in \mathcal{B}(H),
$$

which is a hermitian operator. Note that $W_{T}\left(H_{\alpha}\right) \subseteq H_{\alpha}$ and

$$
W_{T} \mid H_{\alpha}=i\left(U_{T} \mid H_{\alpha}-\left(U_{T} \mid H_{\alpha}\right)^{*}\right)
$$

for all $\alpha$.

Lemma 3.4. If $T \in C_{\mathcal{E}}(\mathcal{D})_{\mathrm{sym}}$, then $T \mid H_{\alpha}=\left(W_{T} \mid H_{\alpha}\right)\left(D_{T} \mid H_{\alpha}\right)^{-1}$ for all $\alpha \in \Lambda$.

Proof. We use the same argument carried out in [25]. Take $x \in H_{\alpha}$. Then $\left(I-U_{T}\right)\left(T \mid H_{\alpha}+i\right) x=T x+i x-T x+i x=2 i x$. But $x=\left(T \mid H_{\alpha}+i\right)^{-1}\left(T \mid H_{\alpha}+i\right) x ;$ therefore

$$
\left(I-U_{T}\right)\left(T \mid H_{\alpha}+i\right) x=2 i\left(T \mid H_{\alpha}+i\right)^{-1}\left(T \mid H_{\alpha}+i\right) x .
$$

Taking into account that $\mathcal{R}\left(T \mid H_{\alpha}+i\right)=H_{\alpha}$, we deduce that

$$
I-U_{T} \mid H_{\alpha}=2 i\left(T \mid H_{\alpha}+i\right)^{-1} \in \mathcal{B}\left(H_{\alpha}\right)
$$


for all $\alpha \in \Lambda$. Thereby

$$
I-U_{\bar{T}}^{*} \mid H_{\alpha}=\left(1-U_{T} \mid H_{\alpha}\right)^{*}=\left(2 i\left(T \mid H_{\alpha}+i\right)^{-1}\right)^{*}=-2 i\left(T \mid H_{\alpha}-i\right)^{-1} .
$$

It follows that

$$
\begin{aligned}
2 I-U_{T}\left|H_{\alpha}-U_{T}^{*}\right| H_{\alpha} & =I-U_{T}\left|H_{\alpha}+I-U_{T}^{*}\right| H_{\alpha}=2 i\left(T \mid H_{\alpha}+i\right)^{-1}-2 i\left(T \mid H_{\alpha}-i\right)^{-1} \\
& =2 i\left(\left(T \mid H_{\alpha}+i\right)^{-1}-\left(T \mid H_{\alpha}-i\right)^{-1}\right) \\
& =2 i\left(T \mid H_{\alpha}+i\right)^{-1}\left(T \mid H_{\alpha}-i-\left(T \mid H_{\alpha}+i\right)\right)\left(T \mid H_{\alpha}-i\right)^{-1} \\
& =4\left(T \mid H_{\alpha}+i\right)^{-1}\left(T \mid H_{\alpha}-i\right)^{-1} \\
& =4\left(1+T^{2} \mid H_{\alpha}\right)^{-1}
\end{aligned}
$$

that is,

$$
D_{T}\left|H_{\alpha}=2-U_{T}\right| H_{\alpha}-U_{T}^{*} \mid H_{\alpha}=4\left(1+T^{2} \mid H_{\alpha}\right)^{-1} .
$$

Further,

$$
\begin{aligned}
U_{T} \mid & H_{\alpha}-U_{T}^{*} \mid H_{\alpha} \\
& =-\left(1-U_{T} \mid H_{\alpha}\right)+\left(1-U_{T}^{*} \mid H_{\alpha}\right)=-2 i\left(T \mid H_{\alpha}+i\right)^{-1}-2 i\left(T \mid H_{\alpha}-i\right)^{-1} \\
& =-2 i\left(\left(T \mid H_{\alpha}+i\right)^{-1}+\left(T \mid H_{\alpha}-i\right)^{-1}\right) \\
& =-2 i\left(T \mid H_{\alpha}+i\right)^{-1}\left(T \mid H_{\alpha}-i+\left(T \mid H_{\alpha}+i\right)\right)\left(T \mid H_{\alpha}-i\right)^{-1} \\
& =-4 i\left(T \mid H_{\alpha}+i\right)^{-1}\left(T \mid H_{\alpha}\right)\left(T \mid H_{\alpha}-i\right)^{-1}=-4 i\left(T \mid H_{\alpha}\right)\left(1+T^{2} \mid H_{\alpha}\right)^{-1} .
\end{aligned}
$$

That is, $U_{T}\left|H_{\alpha}-U_{T}^{*}\right| H_{\alpha}=-4 i\left(T \mid H_{\alpha}\right)\left(1+T^{2} \mid H_{\alpha}\right)^{-1}$, thereupon

$$
W_{T} \mid H_{\alpha}=4\left(T \mid H_{\alpha}\right)\left(1+T^{2} \mid H_{\alpha}\right)^{-1} .
$$

Using (3.3) and (3.4), we derive that

$$
\begin{aligned}
\left(W_{T} \mid H_{\alpha}\right)\left(D_{T} \mid H_{\alpha}\right)^{-1} & =4\left(T \mid H_{\alpha}\right)\left(1+T^{2} \mid H_{\alpha}\right)^{-1} 4^{-1}\left(1+T^{2} \mid H_{\alpha}\right) \\
& =\left(T \mid H_{\alpha}\right)\left(1+T^{2} \mid H_{\alpha}\right)^{-1}\left(1+T^{2} \mid H_{\alpha}\right) \\
& =T \mid H_{\alpha}
\end{aligned}
$$

that is, $\left(W_{T} \mid H_{\alpha}\right)\left(D_{T} \mid H_{\alpha}\right)^{-1}=T \mid H_{\alpha}$.

Corollary 3.1. Let $T \in C_{\mathcal{E}}(\mathcal{D})_{\mathrm{sym}}$. Then $T \geq_{\alpha} 0$ iff $\operatorname{Im}(\lambda) \leq 0$ for all $\lambda \in$ $\sigma\left(U_{T} \mid H_{\alpha}\right)$. In particular, $T \geq 0$ iff $\sigma\left(U_{\bar{T}}\right)$ lies on the lower semicircle of $\mathbb{T}$.

Proof. By Lemma 3.4. $T \mid H_{\alpha}$ belongs to the commutative $C^{*}$-algebra generated by $U_{T} \mid H_{\alpha}$ in $\mathcal{B}\left(H_{\alpha}\right)$. Due to the spectral mapping theorem, we have

$$
\sigma\left(T \mid H_{\alpha}\right)=\left\{\frac{i(\lambda-\bar{\lambda})}{2-\lambda-\bar{\lambda}}: \lambda \in \sigma\left(U_{T} \mid H_{\alpha}\right)\right\}=\left\{\frac{-\operatorname{Im}(\lambda)}{1-\operatorname{Re}(\lambda)}: \lambda \in \sigma\left(U_{T} \mid H_{\alpha}\right)\right\} .
$$

It follows that $\sigma\left(T \mid H_{\alpha}\right) \geq 0$ iff $\operatorname{Im}(\lambda) \leq 0$ for all $\lambda \in \sigma\left(U_{T} \mid H_{\alpha}\right)$.

Finally, using Proposition 3.1, infer that $\sigma\left(U_{\bar{T}}\right)=\overline{\bigcup_{\alpha \in \Lambda} \sigma\left(U_{T} \mid H_{\alpha}\right)}$. Therefore $T \geq 0$ iff $\operatorname{Im}(\lambda) \leq 0$ for all $\lambda \in \bigcup_{\alpha \in \Lambda} \sigma\left(U_{T} \mid H_{\alpha}\right)$. The latter means that $\sigma\left(U_{\bar{T}}\right)$ lies in the lower semicircle of $\mathbb{T}$. 


\section{Noncommutative RATional FUnCtions}

In this section we introduce noncommutative rational functions on an innerproduct space in the pure algebraic context. The fractional space construction proposed in 25] has a quite commutative nature. When we deal with noncommutative fractions it is automatically appeared as a problem with their sums and products, which is the known algebraic phenomenon. A way to handle noncommutative fractions in elements of a Lie algebra has been proposed in [22 and applied to the multivariable spectral theory in [6]. Some tinges of these ideas are proposed below.

Let $\mathcal{D}$ be an inner-product space whose completion is $H$. Put

$$
\mathfrak{B}_{\mathcal{D}}=\left\{T \in \mathcal{B}(H): T(\mathcal{D}) \subseteq \mathcal{D} \text { and } T^{*}(\mathcal{D}) \subseteq \mathcal{D}\right\} .
$$

Obviously, $\mathfrak{B}_{\mathcal{D}}$ is a unital $*$-subalgebra in $\mathcal{B}(H)$. Further, consider the set $\mathfrak{M}_{\mathcal{D}}$ of all self-adjoint elements $m$ from $\mathfrak{B}_{\mathcal{D}}$ such that $m \mid \mathcal{D}: \mathcal{D} \rightarrow \mathcal{D}$ is a bijection; that is,

$$
\mathfrak{M}_{\mathcal{D}}=\left\{m \in \mathfrak{B}_{\mathcal{D}}: m \text { is a } \mathcal{D} \text {-bijection, } \quad m=m^{*}\right\} .
$$

Obviously, $1_{H} \in \mathfrak{M}_{\mathcal{D}}$ and $m n \in \mathfrak{M}_{\mathcal{D}}$ whenever $m, n \in \mathfrak{M}_{\mathcal{D}}$ and $m n=n m$. We say that $\mathfrak{M}_{\mathcal{D}}$ is the set of all denominators in $\mathfrak{B}_{\mathcal{D}}$.

To define a fraction space $\mathfrak{B}_{\mathcal{D}} / \mathfrak{M}_{\mathcal{D}}$ consider the set $\mathfrak{B}_{\mathcal{D}} \times \mathfrak{M}_{\mathcal{D}}$ of all ordered pairs $(T, m), T \in \mathfrak{B}_{\mathcal{D}}, m \in \mathfrak{M}_{\mathcal{D}}$. Put

$$
(T, m) \sim(S, n) \quad \text { if } \quad T(m \mid \mathcal{D})^{-1}=S(n \mid \mathcal{D})^{-1} .
$$

Note that $T(m \mid \mathcal{D})^{-1}$ and $S(n \mid \mathcal{D})^{-1}$ are linear transformations on $\mathcal{D}$; that is, $T(m \mid \mathcal{D})^{-1}, S(n \mid \mathcal{D})^{-1} \in L(\mathcal{D})$. One can easily verify that $\sim$ is an equivalence relation on $\mathfrak{B}_{\mathcal{D}} \times \mathfrak{M}_{\mathcal{D}}$. We use $T / m$ to indicate the equivalence class of $(T, m)$, that is, an element of the quotient set $\mathfrak{B}_{\mathcal{D}} \times \mathfrak{M}_{\mathcal{D}} / \sim$ denoted by $\mathfrak{B}_{\mathcal{D}} / \mathfrak{M}_{\mathcal{D}}$. The set $\mathfrak{B}_{\mathcal{D}} / \mathfrak{M}_{\mathcal{D}}$ is embedded into $L(\mathcal{D})$ by means of the injective mapping

$$
\mathfrak{B}_{\mathcal{D}} / \mathfrak{M}_{\mathcal{D}} \longrightarrow L(\mathcal{D}), \quad T / m \mapsto T(m \mid \mathcal{D})^{-1} \text {. }
$$

We shall identify $\mathfrak{B}_{\mathcal{D}} / \mathfrak{M}_{\mathcal{D}}$ with its range in $L(\mathcal{D})$, and put $T / m=T(m \mid \mathcal{D})^{-1}$. We also write $T$ instead of $T\left(1_{H} \mid \mathcal{D}\right)^{-1}$, where $T \in \mathfrak{B}_{\mathcal{D}}$. Note that if $T \mid \mathcal{D}=0$, then $T=0$ for $T \in \mathcal{B}(H)$. Thus $\mathfrak{B}_{\mathcal{D}} \subseteq \mathfrak{B}_{\mathcal{D}} / \mathfrak{M}_{\mathcal{D}} \subseteq L(\mathcal{D})$.

Take $T / m \in \mathfrak{B}_{\mathcal{D}} / \mathfrak{M}_{\mathcal{D}}$. Set $(T / m)^{*}=(m \mid \mathcal{D})^{-1} T^{*} \mid \mathcal{D} \in L(\mathcal{D})$; recall that $T^{*}(\mathcal{D}) \subseteq \mathcal{D}$. We also set

$$
\left(\mathfrak{B}_{\mathcal{D}} / \mathfrak{M}_{\mathcal{D}}\right)^{*}=\left\{(T / m)^{*}: T / m \in \mathfrak{B}_{\mathcal{D}} / \mathfrak{M}_{\mathcal{D}}\right\} \subseteq L(\mathcal{D}) .
$$

Then

$$
\begin{aligned}
\left\langle(m \mid \mathcal{D})^{-1} x, y\right\rangle & =\left\langle(m \mid \mathcal{D})^{-1} x,(m \mid \mathcal{D})(m \mid \mathcal{D})^{-1} y\right\rangle=\left\langle(m \mid \mathcal{D})^{-1} x, m(m \mid \mathcal{D})^{-1} y\right\rangle \\
& =\left\langle m(m \mid \mathcal{D})^{-1} x,(m \mid \mathcal{D})^{-1} y\right\rangle \\
& =\left\langle x,(m \mid \mathcal{D})^{-1} y\right\rangle
\end{aligned}
$$

for all $x, y \in \mathcal{D}$. It follows that

$$
\begin{aligned}
\langle(T / m) x, y\rangle & =\left\langle T(m \mid \mathcal{D})^{-1} x, y\right\rangle=\left\langle(m \mid \mathcal{D})^{-1} x, T^{*} y\right\rangle \\
& =\left\langle x,(m \mid \mathcal{D})^{-1} T^{*} y\right\rangle=\left\langle x,(T / m)^{*} y\right\rangle
\end{aligned}
$$


for all $x, y \in \mathcal{D}$. Let us consider the full (or the inverse preserving) [4, 1.1.4] subalgebra $C(\mathcal{D})$ in $L(\mathcal{D})$ generated by the set $\left(\mathfrak{B}_{\mathcal{D}} / \mathfrak{M}_{\mathcal{D}}\right) \cup\left(\mathfrak{B}_{\mathcal{D}} / \mathfrak{M}_{\mathcal{D}}\right)^{*}$. Evidently, each $a \in C(\mathcal{D})$ has the adjoint $a^{*} \in C(\mathcal{D})$ with the property $\langle a x, y\rangle=\left\langle x, a^{*} y\right\rangle$ for all $x, y \in \mathcal{D}$. In this sense, $C(\mathcal{D})$ is a unital $*$-algebra called the algebra of noncommutative rational functions over $\mathcal{D}$.

The following simple description of the *-algebra $C(\mathcal{D})$ can be done. Let $r^{(1)}(\mathcal{D})$ be the subalgebra in $L(\mathcal{D})$ generated by the set $\left(\mathfrak{B}_{\mathcal{D}} / \mathfrak{M}_{\mathcal{D}}\right) \cup\left(\mathfrak{B}_{\mathcal{D}} / \mathfrak{M}_{\mathcal{D}}\right)^{*}$. Each element $a \in r^{(1)}(\mathcal{D})$ is a polynomial $p(F)$ in elements of a finite subset $F \subseteq$ $\left(\mathfrak{B}_{\mathcal{D}} / \mathfrak{M}_{\mathcal{D}}\right) \cup\left(\mathfrak{B}_{\mathcal{D}} / \mathfrak{M}_{\mathcal{D}}\right)^{*}$. Therefore $a$ has the adjoint $a^{*} \in r^{(1)}(\mathcal{D})$ with the property $\langle a x, y\rangle=\left\langle x, a^{*} y\right\rangle$ for all $x, y \in \mathcal{D}$. Let us introduce the set

$$
\mathfrak{M}_{\mathcal{D}}^{(2)}=\left\{m \in r^{(1)}(\mathcal{D}): m \text { is a } \mathcal{D} \text {-bijection, } \quad m=m^{*}\right\}
$$

and put $\mathfrak{M}_{\mathcal{D}}^{(1)}=\mathfrak{M}_{\mathcal{D}}$. By $r^{(1)}(\mathcal{D}) / \mathfrak{M}_{\mathcal{D}}^{(2)}$ we denote the set of all linear transformations $a(m \mid \mathcal{D})^{-1}$. Put again $a / m=a(m \mid \mathcal{D})^{-1}$. Evidently, $(a / m)^{*}=(m \mid \mathcal{D})^{-1} a^{*}$ and let

$$
\left(r^{(1)}(\mathcal{D}) / \mathfrak{M}_{\mathcal{D}}^{(2)}\right)^{*}=\left\{(a / m)^{*}: a / m \in r^{(1)}(\mathcal{D}) / \mathfrak{M}_{\mathcal{D}}^{(2)}\right\} .
$$

We have a subalgebra $r^{(2)}(\mathcal{D}) \subseteq L(\mathcal{D})$ generated by the set $\left(r^{(1)}(\mathcal{D}) / \mathfrak{M}_{\mathcal{D}}^{(2)}\right) \cup$ $\left(r^{(1)}(\mathcal{D}) / \mathfrak{M}_{\mathcal{D}}^{(2)}\right)^{*}$. Based upon the inductive argument, one may define an increasing sequence of $*$-subalgebras $r^{(n)}(\mathcal{D}), n \geq 1$, such that

$$
\mathfrak{B}_{\mathcal{D}} \subseteq r^{(1)}(\mathcal{D}) \subseteq r^{(2)}(\mathcal{D}) \subseteq \cdots \subseteq r^{(n)}(\mathcal{D}) \subseteq \cdots .
$$

By its very definition,

$$
C(\mathcal{D})=\bigcup_{n \geq 0} r^{(n)}(\mathcal{D})
$$

where $r^{(0)}(\mathcal{D})=\mathfrak{B}_{\mathcal{D}}$

\section{Multinormed $C^{*}$-ALgebra of noncommutative Rational FunCtions}

To introduce a topological version of the $*$-algebra $C(\mathcal{D})$ proposed in Section 4 we assume that the inner-product space $\mathcal{D}$ is the union space of a quantum domain $\mathcal{E}=\left\{H_{\alpha}: \alpha \in \Lambda\right\}$ in a Hilbert space $H$.

5.1. The $C^{*}$-algebra $C_{\mathcal{E}}^{*}(H)$ and its multinormed completion. Let $\mathfrak{p}=\left\{P_{\alpha}\right.$ : $\alpha \in \Lambda\}$ be the projection net in $\mathcal{B}(H)$ associated with $\mathcal{E}$. As in Section 4 we set

$$
\mathfrak{B}_{\mathcal{E}}=\left\{T \in \mathcal{B}(H): T(\mathcal{E}) \subseteq \mathcal{E} \text { and } T^{*}(\mathcal{E}) \subseteq \mathcal{E}\right\} .
$$

Thus $T\left(H_{\alpha}\right) \subseteq H_{\alpha}$ and $T^{*}\left(H_{\alpha}\right) \subseteq H_{\alpha}$ for all $\alpha \in \Lambda$. Evidently, $\mathfrak{B}_{\mathcal{E}} \subseteq \mathfrak{B}_{\mathcal{D}}$ and $\mathfrak{B}_{\mathcal{E}}$ is the commutant of the net $\mathfrak{p}$ in $\mathcal{B}(H)$. In particular, it is a $C^{*}$-algebra. Furthermore,

$$
\mathfrak{B}_{\mathcal{E}}=C_{\mathcal{E}}^{*}(H),
$$

thanks to Lemma 2.3. Fix an index $\alpha$, and consider the restriction mapping $\pi_{\alpha}: C_{\mathcal{E}}^{*}(H) \rightarrow \mathcal{B}\left(H_{\alpha}\right), \pi_{\alpha}(T)=T \mid H_{\alpha}$, which is a unital $*$-homomorphism. Therefore its range, denoted by $\mathfrak{B}_{\alpha}$, is a $C^{*}$-algebra in $\mathcal{B}\left(H_{\alpha}\right)$. Furthermore, $\|T\|_{\alpha}=\left\|T \mid H_{\alpha}\right\|_{\mathcal{B}\left(H_{\alpha}\right)}$ is a $C^{*}$-seminorm on $C_{\mathcal{E}}^{*}(H)$, and the $C^{*}$-algebra generated by this seminorm is reduced to $\mathfrak{B}_{\alpha}$. That is, $\mathfrak{B}_{\alpha}$ is the completion of the quotient algebra $C_{\mathcal{E}}^{*}(H) / I_{\alpha}$, where $I_{\alpha}=\left\{T \in C_{\mathcal{E}}^{*}(H):\|T\|_{\alpha}=0\right\}$. Moreover, if $\alpha \leq \beta$ for some $\alpha, \beta \in \Lambda$, then there is a canonical $*$-homomorphism $\pi_{\alpha \beta}: \mathfrak{B}_{\beta} \rightarrow \mathfrak{B}_{\alpha}$ 
such that $\pi_{\alpha \beta}\left(T \mid H_{\beta}\right)=T \mid H_{\alpha}$. Thus we have an inverse system $\left\{\mathfrak{B}_{\alpha}, \pi_{\alpha \beta}\right\}$ of $C^{*}$ -

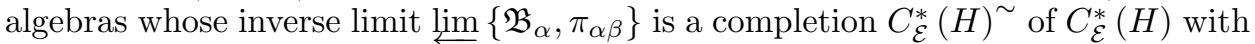
respect to the topology associated by the family of $C^{*}$-seminorms $\left\{\|\cdot\|_{\alpha}: \alpha \in \Lambda\right\}$ (see [15, 5.2.10]).

Definition 5.1. The multinormed $C^{*}$-algebra $C_{\mathcal{E}}^{*}(H)^{\sim}$ is called a topological algebra of noncommutative rational functions on $\mathcal{E}$.

The inclusion $C_{\mathcal{E}}^{*}(H) \subseteq C_{\mathcal{E}}^{*}(H)^{\sim}$ is given by the local contractive $*$-monomorphism

$$
\left(C_{\mathcal{E}}^{*}(H),\|\cdot\|_{\alpha}, \alpha \in \Lambda\right) \rightarrow \lim _{\longleftarrow}\left\{\mathfrak{B}_{\alpha}, \pi_{\alpha \beta}\right\}, \quad T \mapsto\left(T \mid H_{\alpha}\right)_{\alpha \in \Lambda} .
$$

Let us note that if $T \mid H_{\alpha}=0$ for all $\alpha$, then $T \mid \mathcal{D}=0$, and keeping the fact $T \in \mathcal{B}(H)$ in mind, we infer that $T=0$. Thus the $C^{*}$-algebra $C_{\mathcal{E}}^{*}(H)$ has an additional polynormed space structure. Being an inverse limit of the $C^{*}$-algebras, $C_{\mathcal{E}}^{*}(H)^{\sim}$ possesses a canonical quantum space structure. Actually, it is just the completion of the quantum space $C_{\mathcal{E}}^{*}(H)$ whose structure is well defined by the identifications $\mathbb{M}_{k}\left(C_{\mathcal{E}}^{*}(H)\right)=C_{\mathcal{E}^{k}}^{*}\left(H^{k}\right), k \in \mathbb{N}$.

Lemma 5.1. The algebra $C_{\mathcal{E}}^{*}(H)^{\sim}$ can be identified with a certain $*$-subalgebra in $C_{\mathcal{E}}^{*}(\mathcal{D})$ up to a topological $*$-isomorphism.

Proof. Take $b=\left(b_{\alpha}\right)_{\alpha \in \Lambda} \in C_{\mathcal{E}}^{*}(H)^{\sim}=\lim _{\{}\left\{\mathfrak{B}_{\alpha}, \pi_{\alpha \beta}\right\}$. Put $S_{b} x=b_{\alpha} x$ whenever $x \in H_{\alpha}$. If $\alpha \leq \beta$, then $S_{b} x=b_{\alpha} x=\left(b_{\beta} \mid H_{\alpha}\right) x$. So $S_{b} \in L(\mathcal{D})$ is well defined. One can easily verify that $S_{b c}=S_{b} S_{c}$ and $S_{1_{H}}=1_{\mathcal{D}}$. The correspondence $b \mapsto$ $S_{b}$ implements an embedding $C_{\mathcal{E}}^{*}(H)^{\sim} \rightarrow L(\mathcal{D})$. Note that $S_{b}\left(H_{\alpha}\right) \subseteq H_{\alpha}$ and $S_{b} \mid H_{\alpha}=b_{\alpha} \in \mathcal{B}\left(H_{\alpha}\right)$ for all $\alpha$. Thereby, $S_{b} \in C_{\mathcal{E}}(\mathcal{D})$. Furthermore, if $x, y \in H_{\alpha}$, then $\left\langle S_{b} x, y\right\rangle=\left\langle b_{\alpha} x, y\right\rangle=\left\langle x, b_{\alpha}^{*} y\right\rangle=\left\langle x, S_{b^{*}} y\right\rangle$. Thus $S_{b}$ has an unbounded dual $S_{b}^{\star}$ such that $\mathcal{D} \subseteq \mathcal{D}\left(S_{b}^{\star}\right)$ and $S_{b}^{\star} \mid \mathcal{D}=S_{b^{*}} \in C_{\mathcal{E}}(\mathcal{D})$. It follows that $S_{b} \in C_{\mathcal{E}}^{*}(\mathcal{D})$, thanks to [9, Proposition 3.1] (see also Subsection 2.2).

Finally, $\left\|S_{b}\right\|_{\alpha}=\left\|S_{b} \mid H_{\alpha}\right\|=\left\|b_{\alpha}\right\|=\|b\|_{\alpha}$ for all $\alpha$. Consequently, the embedding $C_{\mathcal{E}}^{*}(H)^{\sim} \rightarrow C_{\mathcal{E}}^{*}(\mathcal{D})$ is a topological $*$-isomorphism.

Based upon Lemma 5.1 we shall identify $C_{\mathcal{E}}^{*}(H)^{\sim}$ with its range in $C_{\mathcal{E}}^{*}(\mathcal{D})$. Actually, we shall prove below (see Theorem [5.1) that the inclusion $C_{\mathcal{E}}^{*}(H)^{\sim} \subseteq$ $C_{\mathcal{E}}^{*}(\mathcal{D})$ cannot be proper.

5.2. The fractional spaces. Now let us introduce the set of all denominators $\mathfrak{M}_{\mathcal{E}}$ of the $C^{*}$-algebra $C_{\mathcal{E}}^{*}(H)$ in the following way:

$$
\mathfrak{M}_{\mathcal{E}}=\left\{m \in C_{\mathcal{E}}^{*}(H): m \text { is a } \mathcal{E} \text {-bijection, } \quad m=m^{*}\right\} .
$$

Thus each restriction $m \mid H_{\alpha}$ is invertible in $\mathcal{B}\left(H_{\alpha}\right)$, and $\left(m \mid H_{\alpha}\right)^{-1} \in \mathfrak{B}_{\alpha}$ (see for instance [10, 1.1.12]) for all $\alpha$. In particular, $m$ is a $\mathcal{D}$-bijection too; that is, $m \mid \mathcal{D}: \mathcal{D} \rightarrow \mathcal{D}$ is a bijection. Thereby, $\mathfrak{M}_{\mathcal{E}} \subseteq \mathfrak{M}_{\mathcal{D}}$ (see Section 4). Take $m \in$ $\mathfrak{M}_{\mathcal{E}}$. The family $\left(\left(m \mid H_{\alpha}\right)^{-1}\right)_{\alpha \in \Lambda}$ is compatible in the sense that $\left(m \mid H_{\alpha}\right)^{-1}=$ $\left(m \mid H_{\beta}\right)^{-1} \mid H_{\alpha} \in \mathfrak{B}_{\alpha}$ whenever $\alpha \leq \beta$. Moreover, $(m \mid \mathcal{D})^{-1}=\left(\left(m \mid H_{\alpha}\right)^{-1}\right)_{\alpha \in \Lambda} \in$ $C_{\mathcal{E}}^{*}(H)^{\sim} \subseteq C_{\mathcal{E}}^{*}(\mathcal{D})$ on the grounds of Lemma 5.1. In particular, $T(m \mid \mathcal{D})^{-1} \in$ $C_{\mathcal{E}}^{*}(\mathcal{D})$ whenever $T \in C_{\mathcal{E}}^{*}(H)$. Note also that

$$
T(m \mid \mathcal{D})^{-1}\left|H_{\alpha}=T\left(m \mid H_{\alpha}\right)^{-1}=T\right| H_{\alpha}\left(m \mid H_{\alpha}\right)^{-1} \in \mathcal{B}\left(H_{\alpha}\right),
$$


for all $\alpha$. As in Section 4 , let us introduce an equivalence relation on the set $C_{\mathcal{E}}^{*}(H) \times \mathfrak{M}_{\mathcal{E}}$ of all ordered pairs $(T, m), T \in C_{\mathcal{E}}^{*}(H), m \in \mathfrak{M}_{\mathcal{E}}$, by setting

$$
(T, m) \sim(S, n) \quad \text { if } \quad T(m \mid \mathcal{D})^{-1}=S(n \mid \mathcal{D})^{-1} \quad \text { in } \quad C_{\mathcal{E}}^{*}(\mathcal{D}) .
$$

We put $T / m$ to indicate the equivalence class representing $(T, m)$. Moreover, the quotient set $C_{\mathcal{E}}^{*}(H) \times \mathfrak{M}_{\mathcal{E}} / \sim$ is identified with a subset in $C_{\mathcal{E}}^{*}(\mathcal{D})$ via the identification

$$
C_{\mathcal{E}}^{*}(H) \times \mathfrak{M}_{\mathcal{E}} / \sim \subseteq L(\mathcal{D}), \quad T / m=T(m \mid \mathcal{D})^{-1} .
$$

For each $m \in \mathfrak{M}_{\mathcal{E}}$ we set

$$
C_{\mathcal{E}}^{*}(H) / m=\left\{T / m: T \in C_{\mathcal{E}}^{*}(H)\right\},
$$

which is a subspace in $C_{\mathcal{E}}^{*}(\mathcal{D})$. We say that $C_{\mathcal{E}}^{*}(H) / m$ is a fractional space with the denominator $m$. Thus $\sum_{m \in \mathfrak{M}_{\mathcal{E}}} C_{\mathcal{E}}^{*}(H) / m$ is a subspace in $L(\mathcal{D})$ generated by $C_{\mathcal{E}}^{*}(H) \times \mathfrak{M}_{\mathcal{E}} / \sim$, which is denoted by $C_{\mathcal{E}}^{*}(H) / \mathfrak{M}_{\mathcal{E}}$. Confirm that

$$
1_{\mathcal{D}}=1_{H} / 1_{H}=m / m \in C_{\mathcal{E}}^{*}(H) / m
$$

for all $m \in \mathfrak{M}_{\mathcal{E}}$. In particular, each fractional space $C_{\mathcal{E}}^{*}(H) / m$ is a unital quantum space in $C_{\mathcal{E}}^{*}(\mathcal{D})$.

We equip the set $\mathfrak{M}_{\mathcal{E}}$ of all denominators with a pre-order structure. Namely, set $n \preceq m$ for $n, m \in \mathfrak{M}_{\mathcal{E}}$ if $(n \mid \mathcal{D})^{-1}(m \mid \mathcal{D})$ is bounded. Identifying $(n \mid \mathcal{D})^{-1}(m \mid \mathcal{D})$ with its completion we write $n^{-1} m \in \mathcal{B}(H)$. Moreover, $m n^{-1}$ is also bounded and $\left(n^{-1} m\right)^{*}=m n^{-1}$. Since $n^{-1} m\left(H_{\alpha}\right) \subseteq H_{\alpha}$ and $m n^{-1}\left(H_{\alpha}\right) \subseteq H_{\alpha}$ for all $\alpha$, it follows that $n^{-1} m$ commutes with all projections from the net $\mathfrak{p}$. Whence $n^{-1} m \in \mathfrak{B}_{\mathcal{E}}=C_{\mathcal{E}}^{*}(H)$.

Lemma 5.2. Let $n, m \in \mathfrak{M}_{\mathcal{E}}$. Then $n \preceq m$ iff $C_{\mathcal{E}}^{*}(H) / n \subseteq C_{\mathcal{E}}^{*}(H) / m$.

Proof. If $n^{-1} m$ is bounded and $T \in C_{\mathcal{E}}^{*}(H)$, then we have

$$
\begin{aligned}
T / n & =(T \mid \mathcal{D})(n \mid \mathcal{D})^{-1}=(T \mid \mathcal{D})(n \mid \mathcal{D})^{-1}(m \mid \mathcal{D})(m \mid \mathcal{D})^{-1}=(T \mid \mathcal{D})\left(n^{-1} m \mid \mathcal{D}\right)(m \mid \mathcal{D})^{-1} \\
& =\left(T\left(n^{-1} m\right) \mid \mathcal{D}\right)(m \mid \mathcal{D})^{-1}=\left(T\left(n^{-1} m\right)\right) / m .
\end{aligned}
$$

Hence $C_{\mathcal{E}}^{*}(H) / n \subseteq C_{\mathcal{E}}^{*}(H) / m$.

Conversely, if the latter inclusion holds, then $1_{H} / n=(n \mid \mathcal{D})^{-1}=(S \mid \mathcal{D})(m \mid \mathcal{D})^{-1}$ for a certain $S \in C_{\mathcal{E}}^{*}(H)$. It follows that $n^{-1} m|\mathcal{D}=S| \mathcal{D}$; that is, $n^{-1} m \mid \mathcal{D}$ is bounded.

Let $M \subseteq \mathfrak{M}_{\mathcal{E}}$ be a unital subset of denominators; that is, $1_{H} \in M$. A subset $M_{0} \subseteq M$ is said to be cofinal if for each $n \in M$ there corresponds $m \in M_{0}$ such that $n \preceq m$.

Definition 5.2. Let $M$ be a unital subset in $\mathfrak{M}_{\mathcal{E}}$, and let $\mathcal{F}_{m} \subseteq C_{\mathcal{E}}^{*}(H) / m$ be a subspace for each $m \in M$. An algebraic sum $\mathcal{F}_{M}=\sum_{m \in M} \mathcal{F}_{m}$ of these subspaces is said to be a fractional space if $1_{H} / n \in \mathcal{F}_{n} \subseteq \mathcal{F}_{m}$ whenever $n \preceq m, n, m \in M$.

The sum $\sum_{m \in M} C_{\mathcal{E}}^{*}(H) / m$, denoted by $C_{\mathcal{E}}^{*}(H) / M$, is an example of a fractional space thanks to Lemma 5.2. Another example can be proposed involving a unital $C^{*}$-subalgebra $\mathfrak{J}_{M} \subseteq C_{\mathcal{E}}^{*}(H)$ which contains all $n^{-1} m, n \preceq m, n, m \in M$. In particular, $m=1_{H}^{-1} m \in \mathfrak{J}_{M}$ for each $m \in M$. We say that $\mathfrak{J}_{M}$ is a $C^{*}$-algebra related to the subset $M \subseteq \mathfrak{M}_{\mathcal{E}}$. We again set

$$
\mathfrak{J}_{M} / m=\left\{T / m: T \in \mathfrak{J}_{M}\right\} .
$$


If $n \preceq m$ for some $n, m \in M$, and $T \in \mathfrak{J}_{M}$, then $T\left(n^{-1} m\right) \in \mathfrak{J}_{M}$. Therefore $\mathfrak{J}_{M} / n \subseteq \mathfrak{J}_{M} / m$. Thus $\mathfrak{J}_{M} / M=\sum_{m \in M} \mathfrak{J}_{M} / m$ is a fractional space.

Remark 5.1. Note that $\mathcal{F}_{M}=\sum_{m \in M} \mathcal{F}_{m}=\sum_{m \in M_{0}} \mathcal{F}_{m}=\mathcal{F}_{M_{0}}$ for each cofinal subset $M_{0} \subseteq M$, as follows from Definition 5.2 .

If $T / m \in C_{\mathcal{E}}^{*}(H) / \mathfrak{M}_{\mathcal{E}}$, then we put $(T / m)^{*}=(m \mid \mathcal{D})^{-1} T^{*} \mid \mathcal{D} \in C_{\mathcal{E}}^{*}(\mathcal{D})$ and $\left(C_{\mathcal{E}}^{*}(H) / \mathfrak{M}_{\mathcal{E}}\right)^{*}=\left\{(T / m)^{*}: T / m \in C_{\mathcal{E}}^{*}(H) / \mathfrak{M}_{\mathcal{E}}\right\}$. Thus

$$
\langle(T / m) x, y\rangle=\left\langle x,(T / m)^{*} y\right\rangle
$$

for all $x, y \in \mathcal{D}$ (see Section 4). As in the purely algebraic case, we use $r^{(1)}(\mathcal{E})$ to indicate the subalgebra in $C_{\mathcal{E}}^{*}(\mathcal{D})$ generated by the set $\left(C_{\mathcal{E}}^{*}(H) / \mathfrak{M}_{\mathcal{E}}\right) \cup\left(C_{\mathcal{E}}^{*}(H) / \mathfrak{M}_{\mathcal{E}}\right)^{*}$. This is a $*$-subalgebra in $C_{\mathcal{E}}^{*}(\mathcal{D})$. Replacing $\mathcal{D}$-bijections by $\mathcal{E}$-bijections and using the same argument as in Section 4 one may propose a sequence $r^{(n)}(\mathcal{E}), n \geq 0$, of *subalgebras in $C_{\mathcal{E}}^{*}(\mathcal{D})$ such that $C_{\mathcal{E}}^{*}(H)=r^{(0)}(\mathcal{E}) \subseteq r^{(1)}(\mathcal{E}) \subseteq \cdots \subseteq r^{(n)}(\mathcal{E}) \subseteq \cdots$.

Lemma 5.3. All $r^{(n)}(\mathcal{E})$ are $*$-subalgebras in $C_{\mathcal{E}}^{*}(H)^{\sim}$.

Proof. For $n=0, r^{(0)}(\mathcal{E})=C_{\mathcal{E}}^{*}(H) \subseteq C_{\mathcal{E}}^{*}(H)^{\sim}$ thanks to Lemma 5.1. Assume that $r^{(n-1)}(\mathcal{E}) \subseteq C_{\mathcal{E}}^{*}(H)^{\sim}$ is a $*$-subalgebra. Take a self-adjoint element $b \in r^{(n-1)}(\mathcal{E})$ which is a $\mathcal{E}$-bijection; that is, $b \in \mathfrak{M}_{\mathcal{E}}^{(n)}$ (see Section 4). Thus $b\left(H_{\alpha}\right) \subseteq H_{\alpha}$ and $\left(b \mid H_{\alpha}\right)^{-1} \in \mathcal{B}\left(H_{\alpha}\right)$ for all $\alpha \in \Lambda$. With $C_{\mathcal{E}}^{*}(H)^{\sim}=\varliminf_{\swarrow}\left\{\mathfrak{B}_{\alpha}, \pi_{\alpha \beta}\right\}$ in mind, infer that $b \mid H_{\alpha} \in \mathfrak{B}_{\alpha}$. Then $\left(b \mid H_{\alpha}\right)^{-1} \in \mathfrak{B}_{\alpha}$ for all $\alpha$. Since the family $c=\left(\left(b \mid H_{\alpha}\right)^{-1}\right)_{\alpha \in \Lambda}$ is compatible, we conclude that $c \in C_{\mathcal{E}}^{*}(H)^{\sim}$ (Lemma 5.1) and $c=b^{-1}$. In particular, $a b^{-1} \in C_{\mathcal{E}}^{*}(H)^{\sim}$ for all $a \in r^{(n-1)}(\mathcal{E})$; that is, $r^{(n-1)}(\mathcal{E}) / \mathfrak{M}_{\mathcal{E}}^{(n)} \subseteq C_{\mathcal{E}}^{*}(H)^{\sim}$. Then $\left(r^{(n-1)}(\mathcal{E}) / \mathfrak{M}_{\mathcal{E}}^{(n)}\right)^{*} \subseteq C_{\mathcal{E}}^{*}(H)^{\sim}$, which in turn implies that $r^{(n)}(\mathcal{E}) \subseteq C_{\mathcal{E}}^{*}(H)^{\sim}$.

Remark 5.2. Note that the inclusion $r^{(0)}(\mathcal{E}) \subseteq r^{(1)}(\mathcal{E})$ is proper except in trivial cases. The inverse $(m \mid \mathcal{D})^{-1}$ of a self-adjoint $\mathcal{E}$-bijection $m \in C_{\mathcal{E}}^{*}(H)$ can be discontinuous, which explains the following argument. Take $T \in C_{\mathcal{E}}^{*}(\mathcal{D})_{h}$, which is a symmetric unbounded operator on $H$. Then $D_{T}=2-\bar{U}_{T}-\bar{U}_{T}^{*}$ (see (3.1)) is a positive $\mathcal{E}$-bijection; that is, $D_{T} \in \mathfrak{M}_{\mathcal{E}}$. Moreover, according to (3.3) (see to the proof of Lemma 3.4),

$$
D_{T} \mid H_{\alpha}=4\left(1+T^{2} \mid H_{\alpha}\right)^{-1} \in \mathcal{B}\left(H_{\alpha}\right)
$$

for all $\alpha$. Therefore $\left(D_{T} \mid \mathcal{D}\right)^{-1} \mid H_{\alpha}=\left(D_{T} \mid H_{\alpha}\right)^{-1}=4^{-1}\left(1+T^{2} \mid H_{\alpha}\right)$, whence

$$
\left(D_{T} \mid \mathcal{D}\right)^{-1}=4^{-1}\left(1_{\mathcal{D}}+T^{2}\right) .
$$

The latter in turn implies that $\left(D_{T} \mid \mathcal{D}\right)^{-1}$ is bounded iff $T$ is as well. Indeed, if $T$ is bounded, then $4^{-1}\left(1+T^{2}\right)$ is a bounded operator without any doubt. Conversely, if $\left(D_{T} \mid \mathcal{D}\right)^{-1}$ is bounded, then so is $T^{2}$. Thus $\left\|T \mid H_{\alpha}\right\|^{2}=\left\|\left(T \mid H_{\alpha}\right)^{2}\right\| \leq\left\|T^{2}\right\|$ for all $\alpha$; that is, $\sup \left\{\left\|T \mid H_{\alpha}\right\|\right\}<\infty$. Hence $T$ is bounded. Thus $\left(D_{T} \mid \mathcal{D}\right)^{-1} \in$ $r^{(1)}(\mathcal{E}) \backslash r^{(0)}(\mathcal{E})$ whenever $T$ is not bounded. Moreover, $\left(D_{T} \mid \mathcal{D}\right)^{-1} \in C_{\mathcal{E}}^{*}(H)^{\sim}$ thanks to Lemma 5.3 . 
Theorem 5.1. Let $\mathcal{E}$ be a quantum domain in $H$ with its union space $\mathcal{D}$. Then $r^{(n+1)}(\mathcal{E})=r^{(n)}(\mathcal{E})$ for all $n \geq 1$, and

$$
C_{\mathcal{E}}^{*}(\mathcal{D})=C_{\mathcal{E}}^{*}(H)^{\sim}=r^{(1)}(\mathcal{E})=C_{\mathcal{E}}^{*}(H) / \mathfrak{M}_{\mathcal{E}}
$$

Proof. Using Lemmas 5.1 and 5.3 infer that $\sum_{m \in \mathfrak{M}_{\mathcal{E}}} C_{\mathcal{E}}^{*}(H) / m \subseteq r^{(1)}(\mathcal{E}) \subseteq$ $C_{\mathcal{E}}^{*}(H)^{\sim} \subseteq C_{\mathcal{E}}^{*}(\mathcal{D})$. It remains to prove that $C_{\mathcal{E}}^{*}(\mathcal{D}) \subseteq C_{\mathcal{E}}^{*}(H) / \mathfrak{M}_{\mathcal{E}}$. The canonical expansion of an element from $C_{\mathcal{E}}^{*}(\mathcal{D})$ into its real and imaginary parts allows us to conclude that $C_{\mathcal{E}}^{*}(\mathcal{D})=C_{\mathcal{E}}^{*}(\mathcal{D})_{h}+i C_{\mathcal{E}}^{*}(\mathcal{D})_{h}$, where $C_{\mathcal{E}}^{*}(\mathcal{D})_{h}$ is the set of all hermitian elements in $C_{\mathcal{E}}^{*}(\mathcal{D})$. It suffices to prove that $C_{\mathcal{E}}^{*}(\mathcal{D})_{h} \subseteq C_{\mathcal{E}}^{*}(H) / \mathfrak{M}_{\mathcal{E}}$. Take $T \in C_{\mathcal{E}}^{*}(\mathcal{D})_{h}$. Then $T \in C_{\mathcal{E}}(\mathcal{D})_{\text {sym }}$ (see (2.4)). That is, $T$ is a symmetric unbounded operator on $H$ such that $\mathcal{D}(T)=\mathcal{D}, T\left(H_{\alpha}\right) \subseteq H_{\alpha}$ and $T \mid H_{\alpha} \in \mathcal{B}\left(H_{\alpha}\right)$ for all $\alpha \in \Lambda$. By Theorem 3.1 the Cayley transform $S \mapsto \bar{U}_{S}$ implements a bijection $C_{\mathcal{E}}^{*}(\mathcal{D})_{h} \rightarrow \mathfrak{U}_{\mathcal{E}}$, where $\mathfrak{U}_{\mathcal{E}}$ is the set of all unitary operators $V \in \mathcal{B}(H)$ such that $V\left(H_{\alpha}\right)=H_{\alpha}, \alpha \in \Lambda$, and $1 \notin \bigcup_{\alpha \in \Lambda} \sigma\left(V \mid H_{\alpha}\right)$. Thus $\mathfrak{U}_{\mathcal{E}}$ consists of $\mathcal{E}$-bijections; in particular, $\mathfrak{U}_{\mathcal{E}} \subseteq C_{\mathcal{E}}^{*}(H)$ (see Subsection [5.1). Consider the operators $D_{T}$ and $W_{T}$ determined in (3.1) and (3.2), respectively. As we have just shown in Remark 5.2, $D_{T} \in \mathfrak{M}_{\mathcal{E}}$ for all $T \in C_{\mathcal{E}}^{*}(\mathcal{D})_{h}$. Using Lemma 3.4 infer that $T \mid H_{\alpha}=\left(W_{T} \mid H_{\alpha}\right)\left(D_{T} \mid H_{\alpha}\right)^{-1}$ for all $T \in C_{\mathcal{E}}^{*}(\mathcal{D})_{h}$ and $\alpha$. It follows that $T=A_{T}$, where $A_{T}=W_{T} / D_{T} \in C_{\mathcal{E}}^{*}(H) / D_{T}$. Hence

$$
C_{\mathcal{E}}^{*}(\mathcal{D}) \subseteq \sum_{T \in C_{\mathcal{E}}^{*}(\mathcal{D})_{h}} C_{\mathcal{E}}^{*}(H) / D_{T} \subseteq \sum_{m \in \mathfrak{M}_{\mathcal{E}}} C_{\mathcal{E}}^{*}(H) / m=C_{\mathcal{E}}^{*}(H) / \mathfrak{M}_{\mathcal{E}}
$$

that is, $C_{\mathcal{E}}^{*}(\mathcal{D})=C_{\mathcal{E}}^{*}(H)^{\sim}=r^{(1)}(\mathcal{E})=\sum_{m \in \mathfrak{M}_{\mathcal{E}}} C_{\mathcal{E}}^{*}(H) / m$.

Remark 5.3. As follows from the argument proposed in the proof of Theorem 5.1. $p\left(A_{T}\right)=p(T)$ whenever $T=\left(T_{1}, \ldots, T_{n}\right)$ is an $n$-tuple in $C_{\mathcal{E}}^{*}(\mathcal{D})_{h}, A_{T}=$ $\left(A_{T_{1}}, \ldots, A_{T_{n}}\right), p(T)=\sum_{\tau} c_{\tau} T^{\tau}$ is a noncommutative polynomial in elements of $T$, where $\tau:\{1, \ldots, n\} \rightarrow\{1, \ldots, n\}$ is a mapping and $T^{\tau}=T_{\tau(1)} \cdots T_{\tau(n)}$ is a monomial. In particular,

$$
C_{\mathcal{E}}^{*}(\mathcal{D})=\sum_{T \in C_{\mathcal{E}}^{*}(\mathcal{D})_{h}} C_{\mathcal{E}}^{*}(H) / D_{T}=C_{\mathcal{E}}^{*}(H) / \mathfrak{D},
$$

where $\mathfrak{D}=\left\{D_{T}: T \in C_{\mathcal{E}}^{*}(\mathcal{D})_{h}\right\}$ is a set of positive denominators.

Corollary 5.1. Let $V \subseteq C_{\mathcal{E}}^{*}(\mathcal{D})$ be a quantum system. If $T^{*} T \in V$ for each $T \in V$, then $V$ is a fractional subspace in $C_{\mathcal{E}}^{*}(\mathcal{D})$. In particular, each quantum $*$-algebra is a fractional space.

Proof. Being a quantum system, we have the canonical expansion $V=V_{h}+$ $i V_{h}$. Using Theorem 5.1 and Remark [5.3, we infer that $V \subseteq \sum_{T \in V_{h}} C_{\mathcal{E}}^{*}(H) / D_{T}$ $=C_{\mathcal{E}}^{*}(H) / \mathfrak{D}_{V}$, where $\mathfrak{D}_{V}=\left\{D_{T}: T \in V_{h}\right\}$. Fix $T \in V_{h}$ and let $\mathcal{F}_{T}=V \cap$ $\left(C_{\mathcal{E}}^{*}(H) / D_{T}\right)$ be a subspace in $C_{\mathcal{E}}^{*}(H) / D_{T}$. Note that $T=A_{T}=W_{T} / D_{T} \in V \cap$ $\left(C_{\mathcal{E}}^{*}(H) / D_{T}\right)=\mathcal{F}_{T}$. Therefore $V_{h} \subseteq \sum_{T \in V_{h}} \mathcal{F}_{T}$, which in turn implies that $V=V_{h}+i V_{h} \subseteq \sum_{T \in V_{h}} \mathcal{F}_{T} \subseteq V$; that is, $V=\sum_{T \in V_{h}} \mathcal{F}_{T}$.

If $D_{T} \preceq D_{S}$ for some $T, S \in V_{h}$, then $C_{\mathcal{E}}^{*}(H) / D_{T} \subseteq C_{\mathcal{E}}^{*}(H) / D_{T}$ by virtue of Lemma 5.2 It follows that $\mathcal{F}_{T} \subseteq \mathcal{F}_{S}$. It remains to prove that $1_{H} / D_{T} \in \mathcal{F}_{T}$ (see Definition 5.2). Due to Remark 5.2 we have $1_{H} / D_{T}=\left(D_{T} \mid \mathcal{D}\right)^{-1}=4^{-1}\left(1_{\mathcal{D}}+T^{2}\right)$. By assumption $V$ is a quantum system with $S^{*} S \in V$ for all $S \in V$. In particular, $1_{\mathcal{D}} \in V$ and $T^{2} \in V$ for all $T \in V_{h}$. Thus $1_{H} / D_{T} \in V \cap C_{\mathcal{E}}^{*}(H) / D_{T}=\mathcal{F}_{T}$. 
5.3. Normal algebras of unbounded operators. The theory of normal algebras consisting of unbounded operators has been developed in [25] by F.-H. Vasilescu. Fix a dense subspace $\mathcal{D}$ in $H$ and consider the unital $*$-algebra

$$
L^{*}(\mathcal{D})=\left\{T \in L(\mathcal{D}): \mathcal{D} \subseteq \mathcal{D}\left(T^{\star}\right), T^{\star}(\mathcal{D}) \subseteq \mathcal{D}\right\}
$$

of unbounded operators with the involution $T \mapsto T^{*}=T^{\star} \mid \mathcal{D}$. A unital *subalgebra $\mathcal{N} \subseteq L^{*}(\mathcal{D})$ is said to be a normal algebra if the closure $\bar{T}$ of each $T \in \mathcal{N}$ is a normal unbounded operator on $H$. Being a unital $*$-algebra, every normal algebra is generated by its hermitian elements.

Proposition 5.1. A unital *-algebra is a normal algebra if and only if it is a unital commutative quantum *-algebra on a certain quantum domain up to a unital *-isomorphism.

Proof. Let $\mathcal{N}$ be a normal algebra in $L^{*}(\mathcal{D})$ and let $A=\left(A_{\iota}\right)_{\iota \in \mathcal{I}}$ be a family of hermitian generators of the algebra $\mathcal{N}$. So, $\mathcal{N}=\left\{p(A): p \in \mathcal{P}_{\mathcal{I}}\right\}$, where $\mathcal{P}_{\mathcal{I}}$ is the $*$-algebra of all complex polynomials in $\mathcal{I}$-real variables. One of the central results of the paper [25] asserts that $\mathcal{N}$ can be identified with a $*$-subalgebra of the algebra of fractions on a compact subset $\Omega$ of $\mathbb{T}^{\mathcal{I}}$. Namely, each $A_{\iota}$ is identified with the fraction $\theta_{\iota}(z)=-\operatorname{Im}\left(z_{\iota}\right) /\left(1-\operatorname{Re}\left(z_{\iota}\right)\right), z \in \Omega, \iota \in \mathcal{I}$. Consequently, $\mathcal{N}$ is identified with the set of all rational functions like $p \circ \theta$, where $p \in \mathcal{P}_{\mathcal{I}}$, $\theta=\left(\theta_{\iota}\right)_{\iota \in \mathcal{I}}$. In fact, $\mathcal{N}$ has a realization as polynomial functions $p(t)$ over a certain "unbounded" set $S \subseteq \mathbb{R}^{\mathcal{I}}$; that is, $\mathcal{N}=\mathcal{P}_{\mathcal{I}}(S)$ up to a unital $*$-isomorphism. Note that, in general, $S$ is not a locally compact space. Consider the Hilbert space $H=\ell_{2}(S)$ of all square summable families $x=\left(x_{s}\right)_{s \in S}$ of complex numbers with the inner product $\langle x, y\rangle=\sum_{s \in S} x_{s} \overline{y_{s}}$. For each compact (in particular, finite) subset $K \subseteq S$ we have a closed subspace $H_{K}=\left\{x \in H: x_{s}=0, s \notin K\right\}$ in $H$. If $G \subseteq K$, then $H_{G} \subseteq H_{K}$. That is, we deal with an upward filtered family $\mathcal{E}=\left\{H_{K}\right\}$ of closed subspaces in $H$ whose union is denoted by $\mathcal{D}$. Take $x \in H$. Then $\|x\|^{2}=\sum_{s \in S}\left|x_{s}\right|^{2}=\sup \left\{\sum_{s \in F}\left|x_{s}\right|^{2}: F \subseteq S\right\}$, which in turn implies that $x_{s}=0$ for all $s \in S$ except the points of a certain countable subset $C \subseteq S$. Indeed, $\lim _{n} \sum_{s \in F_{n}}\left|x_{s}\right|^{2}=\|x\|^{2}$ for a certain sequence $\left\{F_{n}\right\}$ of finite subsets in $S$. If $t \notin \bigcup_{n} F_{n}$, then again $\lim _{n}\left(\left|x_{t}\right|^{2}+\sum_{s \in F_{n}}\left|x_{s}\right|^{2}\right)=\|x\|^{2}$, which means that $\left|x_{t}\right|=0$. Thus $x=\sum_{n \in \mathbb{N}} x_{s_{n}} s_{n}$ for a certain countable subset $\left(s_{n}\right) \subseteq S$, and $\|x\|^{2}=$ $\sum_{n}\left|x_{s_{n}}\right|^{2}$. Put $y_{n}=\sum_{k \leq n} x_{s_{k}} s_{k} \in \mathcal{D}, n \in \mathbb{N}$. Then $\left\|x-y_{n}\right\|^{2}=\sum_{k>n}\left|x_{s_{k}}\right|^{2} \rightarrow 0$ as $n \rightarrow \infty$. It follows that $\mathcal{D}$ is dense in $H$, which means that $\mathcal{E}$ is a quantum domain in $H$ with its union space $\mathcal{D}$. Moreover, the polynomial algebra $\mathcal{P}_{\mathcal{I}}(S)$ can be embedded into the multinormed $C^{*}$-algebra $C_{\mathcal{E}}^{*}(\mathcal{D})$ by means of the unital *-homomorphism

$$
\mathcal{P}_{\mathcal{I}}(S) \rightarrow C_{\mathcal{E}}^{*}(\mathcal{D}), \quad p(t) \mapsto T_{p}, \quad T_{p}\left(\left(x_{s}\right)_{s \in S}\right)=\left(p(s) x_{s}\right)_{s \in S} .
$$

If $x=\sum_{s \in K} x_{s} s \in H_{K}$, then $T_{p} x=\sum_{s \in K} p(s) x_{s} s$ and

$$
\left\|T_{p} x\right\|^{2}=\sum_{s \in K}\left|p(s) x_{s}\right|^{2} \leq \sup \left\{|p(s)|^{2}: s \in K\right\}\|x\|^{2} ;
$$

that is,

$$
\left\|T_{p}\left|H_{K}\|\leq\| p\right|_{K}\right\|_{\infty} .
$$

Hence $\mathcal{P}_{\mathcal{I}}(S)$ is identified with a commutative unital quantum $*$-algebra in $C_{\mathcal{E}}^{*}(\mathcal{D})$. 
Now assume that $V \subseteq C_{\mathcal{E}}^{*}(\mathcal{D})$ is a unital commutative quantum $*$-algebra on a quantum domain $\mathcal{E}=\left\{H_{\alpha}\right\}_{\alpha \in \Lambda}$ with its union space $\mathcal{D}$. Evidently, $C_{\mathcal{E}}^{*}(\mathcal{D}) \subseteq L^{*}(\mathcal{D})$. Let us prove that $V$ is a normal algebra in $L^{*}(\mathcal{D})$. Take $T \in V$. Then $T^{*} \in V$ and $T T^{*}=T^{*} T$, for $V$ is commutative. It follows that $V$ consists of normal elements. Using Proposition 3.3, we derive that the closure $\bar{T}$ of each $T \in V$ is a normal unbounded operator. Whence $V$ is a normal algebra.

\section{ThE FRACTIONAL MATRIX TOPOLOGY AND FRACTIONAL POSITIVITY}

In this section we introduce the fractional matrix topology and fractional positivity in the multinormed $C^{*}$-algebra $C_{\mathcal{E}}^{*}(\mathcal{D})$ based upon Theorem 5.1 .

6.1. The fractional matrix topology. Let $\mathcal{E}=\left\{H_{\alpha}: \alpha \in \Lambda\right\}$ be a domain in a Hilbert space $H$ with its union space $\mathcal{D}$. Fix a denominator $m \in \mathfrak{M}_{\mathcal{E}}$, which is a self-adjoint $\mathcal{E}$-bijection from $C_{\mathcal{E}}^{*}(H)$. Actually, it can be assumed that $m \geq 0$ (see Remark 5.3).

Lemma 6.1. The fractional space $C_{\mathcal{E}}^{*}(H) / m$ is dense in $C_{\mathcal{E}}^{*}(\mathcal{D})$.

Proof. By Theorem 5.1 $C_{\mathcal{E}}^{*}(H)$ is a dense subspace in $C_{\mathcal{E}}^{*}(\mathcal{D})$. If $S \in C_{\mathcal{E}}^{*}(\mathcal{D})$, then $S=\lim \left\{T_{j}\right\}$ for a certain net $\left\{T_{j}\right\}$ in $C_{\mathcal{E}}^{*}(H)$. Thus $\lim _{j}\left\|S\left|H_{\alpha}-T_{j}\right| H_{\alpha}\right\|_{\mathcal{B}\left(H_{\alpha}\right)}=0$ for all $\alpha$. Put $S_{j}=\left(T_{j} m\right) / m \in C_{\mathcal{E}}^{*}(H) / m$. Then

$$
\begin{aligned}
\lim _{j}\left\|S\left|H_{\alpha}-S_{j}\right| H_{\alpha}\right\|_{\mathcal{B}\left(H_{\alpha}\right)} & =\lim _{j}\left\|S \mid H_{\alpha}-T_{j}\left(m \mid H_{\alpha}\right)\left(m \mid H_{\alpha}\right)^{-1}\right\|_{\mathcal{B}\left(H_{\alpha}\right)} \\
& =\lim _{j}\left\|S\left|H_{\alpha}-T_{j}\right| H_{\alpha}\right\|_{\mathcal{B}\left(H_{\alpha}\right)} \\
& =0 ;
\end{aligned}
$$

that is, $S=\lim \left\{S_{j}\right\}$. Therefore $C_{\mathcal{E}}^{*}(H) / m$ is dense in $C_{\mathcal{E}}^{*}(\mathcal{D})$.

Each fractional space $C_{\mathcal{E}}^{*}(H) / m$ possesses another matrix norm-topology inherited from $C_{\mathcal{E}}^{*}(H)$.

Lemma 6.2. Let $b \in C_{\mathcal{E}}^{*}(\mathcal{D})$. The following conditions are equivalent:

(i) $b \in C_{\mathcal{E}}^{*}(H) / m$,

(ii) $m b^{*}$ is bounded,

(iii) bm is bounded.

Proof. The equivalence $(i) \Leftrightarrow($ iii $)$ is clear. However, the equivalence $(i) \Leftrightarrow(i i i)$ follows from the relations $(m \mid \mathcal{D})^{*}(b \mid \mathcal{D})^{*} \subseteq(b m \mid \mathcal{D})^{*}$ and $\left(b^{*} \mid \mathcal{D}\right)^{*}(m \mid \mathcal{D})^{*} \subseteq\left(m b^{*} \mid \mathcal{D}\right)^{*}$ 20, 13.13.2].

We use the same denotation $b m$ for the bounded extension of $b m$ whenever $b \in C_{\mathcal{E}}^{*}(H) / m$. Thus $b m \in C_{\mathcal{E}}^{*}(H)$. Let us introduce the norm on the fractional space $C_{\mathcal{E}}^{*}(H) / m$ by the rule

$$
\|b\|_{m}=\|b m\|_{\mathcal{B}(H)}=\left\|m b^{*}\right\|_{\mathcal{B}(H)}
$$

which is well defined thanks to Lemma 6.2 We also introduce the seminorms

$$
\|b\|_{m, \alpha}=\left\|(b m)\left|H_{\alpha}\left\|_{\mathcal{B}\left(H_{\alpha}\right)}=\right\|\left(m b^{*}\right)\right| H_{\alpha}\right\|_{\mathcal{B}\left(H_{\alpha}\right)},
$$

where $\alpha \in \Lambda$. The space $C_{\mathcal{E}}^{*}(H) / m$ equipped with the seminorms $\|\cdot\|_{m, \alpha}$ is a (Hausdorff) polynormed space such that $\|\cdot\|_{m}=\sup \left\{\|\cdot\|_{m, \alpha}: \alpha \in \Lambda\right\}$; that is, 
$\|\cdot\|_{m}$ is a dominating norm. All these seminorms can be lifted to the matrices over $C_{\mathcal{E}}^{*}(\mathcal{D})$. First, note that

$$
\mathbb{M}_{k}\left(C_{\mathcal{E}}^{*}(H) / m\right) \subseteq \mathbb{M}_{k}\left(C_{\mathcal{E}}^{*}(\mathcal{D})\right)=C_{\mathcal{E}^{k}}\left(\mathcal{D}^{k}\right),
$$

where $\mathcal{E}^{k}=\left\{H_{\alpha}^{k}: \alpha \in \Lambda\right\}$ is a domain in $H^{k}$ with its union space $\mathcal{D}^{k}$. If $e=$ $m \cdot 1_{H^{k}} \in C_{\mathcal{E}^{k}}^{*}\left(H^{k}\right)$, then $e \in \mathfrak{M}_{\mathcal{E}^{k}}$; that is, $e$ is a denominator in $C_{\mathcal{E}^{k}}^{*}\left(H^{k}\right)$. Moreover,

$$
\mathbb{M}_{k}\left(C_{\mathcal{E}}^{*}(H) / m\right)=C_{\mathcal{E}^{k}}^{*}\left(H^{k}\right) /\left(m \cdot 1_{H^{k}}\right)
$$

Indeed,

$$
\begin{aligned}
{\left[T_{i j} / m\right] } & =\left[T_{i j}(m \mid \mathcal{D})^{-1}\right]=\left[T_{i j}\right]\left[\begin{array}{lll}
(m \mid \mathcal{D})^{-1} & & 0 \\
& \ddots & \\
& & (m \mid \mathcal{D})^{-1}
\end{array}\right] \\
& =\left[T_{i j}\right]\left(\left(m \cdot 1_{H^{k}}\right) \mid \mathcal{D}^{k}\right)^{-1} \\
& =\left[T_{i j}\right] /\left(m \cdot 1_{H^{k}}\right)
\end{aligned}
$$

for all $\left[T_{i j}\right] \in \mathbb{M}_{k}\left(C_{\mathcal{E}}^{*}(H) / m\right)$. In particular, $\mathbb{M}_{k}\left(\mathfrak{J}_{M} / m\right)=\mathbb{M}_{k}\left(\mathfrak{J}_{M}\right) /\left(m \cdot 1_{H^{k}}\right)$ for a $C^{*}$-subalgebra $\mathfrak{J}_{M} \subseteq C_{\mathcal{E}}^{*}(H)$ related to a unital subset $M \subseteq \mathfrak{M}_{\mathcal{E}}$. Moreover,

$$
\mathbb{M}_{k}\left(\mathfrak{J}_{M} / M\right)=\sum_{m \in M} \mathbb{M}_{k}\left(\mathfrak{J}_{M} / m\right)=\sum_{e \in M^{k}} \mathbb{M}_{k}\left(\mathfrak{J}_{M}\right) / e \subseteq \mathbb{M}_{k}\left(C_{\mathcal{E}}^{*}(\mathcal{D})\right)
$$

and

$$
\mathbb{M}_{k}\left(C_{\mathcal{E}}^{*}(\mathcal{D})\right)=C_{\mathcal{E}^{k}}\left(\mathcal{D}^{k}\right)=\sum_{e \in C_{\mathcal{E}^{k}}\left(\mathcal{D}^{k}\right)_{h}} C_{\mathcal{E}^{k}}^{*}\left(H^{k}\right) / e=\sum_{e \in \mathfrak{M}_{\mathcal{E}^{k}}} C_{\mathcal{E}^{k}}^{*}\left(H^{k}\right) / e,
$$

due to Theorem 5.1 where $M^{k}=\left\{m \cdot 1_{H^{k}}: m \in M\right\}$. If $b \in \mathbb{M}_{k}\left(C_{\mathcal{E}}^{*}(H) / m\right)$, then we put

$$
\|b\|_{m, \alpha}^{(k)}=\left\|\left(b\left(m \cdot 1_{H^{k}}\right)\right)\left|H_{\alpha}^{k}\left\|_{\mathcal{B}\left(H_{\alpha}^{k}\right)}=\right\|\left(\left(m \cdot 1_{H^{k}}\right) b^{*}\right)\right| H_{\alpha}^{k}\right\|_{\mathcal{B}\left(H_{\alpha}^{k}\right)},
$$

which is well defined thanks to (6.2) and Lemma 6.2. Obviously, $\|\cdot\|_{m, \alpha}^{(1)}=\|\cdot\|_{m, \alpha}$, $\alpha \in \Lambda$.

Lemma 6.3. The family $q_{m, \alpha}=\left\{\|\cdot\|_{m, \alpha}^{(k)}: k \in \mathbb{N}\right\}$ is a matrix seminorm on $C_{\mathcal{E}}^{*}(H) / m$ for all $\alpha \in \Lambda$. The fractional space $C_{\mathcal{E}}^{*}(H) / m$ equipped with the $m a-$ trix seminorms $\left\{q_{m, \alpha}: \alpha \in \Lambda\right\}$ turns out to be a quantum space. Moreover, $q_{m}=$ $\sup \left\{q_{m, \alpha}: \alpha \in \Lambda\right\}$ is a matrix norm on $C_{\mathcal{E}}^{*}(H) / m$.

Proof. Let us verify that $q_{m, \alpha}$ possesses the properties stated in the axioms M1 and M2 (see Section 2). Take $b \in \mathbb{M}_{k}\left(C_{\mathcal{E}}^{*}(H) / m\right), c \in \mathbb{M}_{s}\left(C_{\mathcal{E}}^{*}(H) / m\right), \lambda \in \mathbb{M}_{s, k}$ and $\mu \in \mathbb{M}_{k, s}$. Using (6.2), infer that $b=T /\left(m \cdot 1_{H^{k}}\right)$ and $c=S /\left(m \cdot 1_{H^{s}}\right)$ for some $T \in C_{\mathcal{E}^{k}}^{*}\left(H^{k}\right)$ and $S \in C_{\mathcal{E}^{s}}^{*}\left(H^{s}\right)$. Then

$$
\begin{aligned}
\|b \oplus c\|_{m, \alpha}^{(k+s)} & =\left\|(T \oplus S) /\left(m \cdot 1_{H^{k+s}}\right)\right\|_{m, \alpha}^{(k+s)}=\left\|(T \oplus S) \mid H_{\alpha}^{k+s}\right\|_{\mathcal{B}\left(H_{\alpha}^{k+s}\right)} \\
& =\left\|\left(T \mid H_{\alpha}^{k}\right) \oplus\left(S \mid H_{\alpha}^{s}\right)\right\|_{\mathcal{B}\left(H_{\alpha}^{k+s}\right)}=\max \left\{\left\|T\left|H_{\alpha}^{k}\left\|_{\mathcal{B}\left(H_{\alpha}^{k}\right)},\right\| S\right| H_{\alpha}^{s}\right\|_{\mathcal{B}\left(H_{\alpha}^{s}\right)}\right\} \\
& =\max \left\{\|b\|_{m, \alpha}^{(k)},\|c\|_{m, \alpha}^{(s)}\right\},
\end{aligned}
$$


so we have M1. Further,

$$
\begin{aligned}
\|\lambda b \mu\|_{m, \alpha}^{(s)} & =\left\|(\lambda T \mu) /\left(m \cdot 1_{H^{s}}\right)\right\|_{m, \alpha}^{(s)}=\left\|(\lambda T \mu) \mid H_{\alpha}^{s}\right\|_{\mathcal{B}\left(H_{\alpha}^{s}\right)}=\left\|\lambda\left(T \mid H_{\alpha}^{k}\right) \mu\right\|_{\mathcal{B}\left(H_{\alpha}^{s}\right)} \\
& \leq\|\lambda\|\left\|T \mid H_{\alpha}^{k}\right\|_{\mathcal{B}\left(H_{\alpha}^{k}\right)}\|\mu\|=\|\lambda\|\|b\|_{m, \alpha}^{(k)}\|\mu\| ;
\end{aligned}
$$

that is, M2 is satisfied. Consequently, each $q_{m, \alpha}$ is a matrix seminorm. Thus $C_{\mathcal{E}}^{*}(H) / m$ is a quantum space equipped with the matrix seminorms $\left\{q_{m, \alpha}: \alpha \in \Lambda\right\}$.

Finally, put $q_{m}=\left\{\|\cdot\|_{m}^{(k)}: k \in \mathbb{N}\right\}$, where $\|b\|_{m}^{(k)}=\left\|b\left(m \cdot 1_{H^{k}}\right)\right\|_{\mathcal{B}\left(H^{k}\right)}, b \in$ $\mathbb{M}_{k}\left(C_{\mathcal{E}}^{*}(H) / m\right)$. On the same grounds $q_{m}$ is a matrix norm. Moreover, $q_{m}=$ $\sup \left\{q_{m, \alpha}: \alpha \in \Lambda\right\}$.

Thus $q_{m}$ is a dominating matrix norm of the quantum space $C_{\mathcal{E}}^{*}(H) / m$. We say that $C_{\mathcal{E}}^{*}(H) / m$, equipped with the matrix norm $q_{m}$, is a dominating normed quantum space.

Lemma 6.4. The dominating normed quantum space $C_{\mathcal{E}}^{*}(H) / m$ is complete, and the linear mapping

$$
\theta_{m}: C_{\mathcal{E}}^{*}(H) \rightarrow C_{\mathcal{E}}^{*}(H) / m, \quad \theta_{m}(T)=T / m,
$$

is an isometrical matrix isomorphism of the relevant quantum spaces with respect to their fixed families of matrix seminorms. It also implements a matrix isometry of the $C^{*}$-algebra $C_{\mathcal{E}}^{*}(H)$ onto the dominating normed quantum space $C_{\mathcal{E}}^{*}(H) / \mathrm{m}$.

Proof. If $\left\{b_{n}\right\}$ is a Cauchy sequence in $C_{\mathcal{E}}^{*}(H) / m$, then $\lim _{n} b_{n} m=T$ in $\mathcal{B}(H)$ for some $T \in \mathcal{B}(H)$. Put $b=T / m \in C_{\mathcal{E}}^{*}(H) / m$. Then

$$
\left\|b-b_{n}\right\|_{m}=\left\|\left(b-b_{n}\right) m\right\|_{\mathcal{B}(H)}=\left\|b m-b_{n} m\right\|_{\mathcal{B}(H)}=\left\|T-b_{n} m\right\|_{\mathcal{B}(H)} \rightarrow 0, \quad n \rightarrow \infty ;
$$

that is, $\lim _{n} b_{n}=b$ in $C_{\mathcal{E}}^{*}(H) / m$. Thus $C_{\mathcal{E}}^{*}(H) / m$ is a Banach space.

The linear mapping $C_{\mathcal{E}}^{*}(H) \rightarrow C_{\mathcal{E}}^{*}(H) / m, T \mapsto T / m$, is an isomorphism, for if $T / m=0$, then $T(m \mid \mathcal{D})^{-1}=0$ or $T \mid \mathcal{D}=0$, which in turn implies that $T=0$. Fix $\alpha \in \Lambda$ and take $T=\left[T_{i j}\right] \in \mathbb{M}_{k}\left(C_{\mathcal{E}}^{*}(H)\right)=C_{\mathcal{E}^{k}}^{*}\left(H^{k}\right)$. Then $\theta_{m}^{(k)}(T)=\left[\theta_{m}\left(T_{i j}\right)\right]=\left[T_{i j} / m\right]=T /\left(m \cdot 1_{H^{k}}\right)$ by virtue of (6.2). Furthermore, $q_{m, \alpha}^{(k)}\left(\theta_{m}^{(k)}(T)\right)=\left\|T \mid H_{\alpha}^{k}\right\|_{\mathcal{B}\left(H_{\alpha}^{k}\right)}=\|T\|_{\alpha}^{(k)}$. Thus $\theta_{m}$ is a matrix isometry with respect to the indicated families of matrix seminorms.

Finally, $\left\|\theta_{m}^{(k)}(T)\right\|_{m}^{(k)}=\sup _{\alpha}\left\{q_{m, \alpha}^{(k)}\left(\theta_{m}^{(k)}(T)\right)\right\}=\sup _{\alpha}\left\{\|T\|_{\alpha}^{(k)}\right\}=\|T\|_{\mathcal{B}\left(H^{k}\right)}$ for all $T \in C_{\mathcal{E}^{k}}^{*}\left(H^{k}\right)$; that is, $\theta_{m}: C_{\mathcal{E}}^{*}(H) \rightarrow C_{\mathcal{E}}^{*}(H) / m, T \mapsto T / m$, is a matrix isometry of the dominating normed quantum spaces.

If $n \preceq m$ for some $n, m \in \mathfrak{M}_{\mathcal{E}}$, then $n^{-1} m$ is bounded and

$$
\left(n \cdot 1_{H^{k}}\right)^{-1}\left(m \cdot 1_{H^{k}}\right)=\left(n^{-1} \cdot 1_{H^{k}}\right)\left(m \cdot 1_{H^{k}}\right)=n^{-1} m \cdot 1_{H^{k}} .
$$

Therefore $n^{-1} m \cdot 1_{H^{k}}$ is bounded too. Hence $\left(n \cdot 1_{H^{k}}\right) \preceq\left(m \cdot 1_{H^{k}}\right)$. Using (6.2) and Lemma 5.2, infer that $C_{\mathcal{E}^{k}}^{*}\left(H^{k}\right) /\left(n \cdot 1_{H^{k}}\right) \subseteq C_{\mathcal{E}^{k}}^{*}\left(H^{k}\right) /\left(m \cdot 1_{H^{k}}\right)$.

Lemma 6.5. The inclusion $C_{\mathcal{E}}^{*}(H) / n \subseteq C_{\mathcal{E}}^{*}(H) / m$ is a matrix continuous mapping of the relevant quantum spaces. It is also a matrix bounded embedding between the relevant dominating normed quantum spaces. 
Proof. Take $b=T /\left(n \cdot 1_{H^{k}}\right)$. Then $n^{-1} m$ is bounded and

$$
b=\left(T\left(n^{-1} m \cdot 1_{H^{k}}\right)\right) /\left(m \cdot 1_{H^{k}}\right) \in C_{\mathcal{E}^{k}}^{*}\left(H^{k}\right) /\left(m \cdot 1_{H^{k}}\right) .
$$

Moreover,

$$
\begin{aligned}
\|b\|_{m, \alpha}^{(k)} & =\left\|\left(T\left(n^{-1} m \cdot 1_{H^{k}}\right)\right) \mid H_{\alpha}^{k}\right\|_{\mathcal{B}\left(H_{\alpha}^{k}\right)}=\left\|\left(T \mid H_{\alpha}^{k}\right)\left(\left(n^{-1} m \cdot 1_{H^{k}}\right) \mid H_{\alpha}^{k}\right)\right\|_{\mathcal{B}\left(H_{\alpha}^{k}\right)} \\
& \leq\left\|T \mid H_{\alpha}^{k}\right\|_{\mathcal{B}\left(H_{\alpha}\right)}\left\|\left(\left(n^{-1} m\right) \mid H_{\alpha} \cdot 1_{H_{\alpha}^{k}}\right)\right\|_{\mathcal{B}\left(H_{\alpha}^{k}\right)} \\
& =\|b\|_{n, \alpha}^{(k)}\left\|\left(n^{-1} m\right) \mid H_{\alpha}\right\|_{\mathcal{B}\left(H_{\alpha}\right)} .
\end{aligned}
$$

Thus $\|\cdot\|_{m, \alpha}^{(k)} \leq\left\|\left(n^{-1} m\right) \mid H_{\alpha}\right\|_{\mathcal{B}\left(H_{\alpha}\right)}\|\cdot\|_{n, \alpha}^{(k)}$, which in turn implies that (see Lemma 6.3)

$$
q_{m, \alpha} \leq\left\|\left(n^{-1} m\right) \mid H_{\alpha}\right\|_{\mathcal{B}\left(H_{\alpha}\right)} q_{n, \alpha} .
$$

In particular, $q_{m} \leq\left\|n^{-1} m\right\|_{\mathcal{B}(H)} q_{n}$.

Now let $M \subseteq \mathfrak{M}_{\mathcal{E}}$ be a unital subset of denominators and consider the fractional space $C_{\mathcal{E}}^{*}(H) / M=\sum_{m \in M} C_{\mathcal{E}}^{*}(H) / m$ in $C_{\mathcal{E}}^{*}(\mathcal{D})$ of all rational functions whose denominators belong to $M$. Being an algebraic sum of the dominating normed quantum spaces, $C_{\mathcal{E}}^{*}(H) / M$ can be equipped with the inductive matrix (or local operator) topology [13, Section 8] such that all inclusions $C_{\mathcal{E}}^{*}(H) / m \rightarrow C_{\mathcal{E}}^{*}(H) / M$, $m \in M$, are matrix continuous. We say that this is a fractional matrix topology on $C_{\mathcal{E}}^{*}(H) / M$. Thus a linear mapping $\varphi: C_{\mathcal{E}}^{*}(H) / M \rightarrow X$ into a quantum space $X$ is matrix continuous if and only if so are all restrictions $\varphi_{m}: C_{\mathcal{E}}^{*}(H) / m \rightarrow X$, $\varphi_{m}(b)=\varphi(b), m \in M$. If $n \preceq m$ for some $n, m \in M$, then $C_{\mathcal{E}}^{*}(H) / n \subseteq C_{\mathcal{E}}^{*}(H) / m$ by virtue of Lemma 5.2 , and $\varphi_{n}=\varphi_{m} \mid C_{\mathcal{E}}^{*}(H) / n$. Since the embedding $C_{\mathcal{E}}^{*}(H) / n \subseteq$ $C_{\mathcal{E}}^{*}(H) / m$ is a matrix bounded mapping between the dominating normed quantum spaces (Lemma 6.5), it follows that $\varphi_{n}$ is matrix continuous whenever $\varphi_{m}$ is as well. In particular, the fractional matrix topologies on $C_{\mathcal{E}}^{*}(H) / M$ and $C_{\mathcal{E}}^{*}(H) / M_{0}$ are the same for each cofinal subset $M_{0} \subseteq M$. Furthermore, each fractional space $\mathcal{F}_{M}=$ $\sum_{m \in M} \mathcal{F}_{m}$ (see Definition 5.2) being an inductive limit of the normed quantum spaces possesses the fractional matrix topology. Consequently, a quantum $*$-algebra can be equipped with a fractional matrix topology thanks to Corollary 5.1. Finally, a linear functional $\varphi: \mathcal{F}_{M} \rightarrow \mathbb{C}$ is said to be fractionally $\alpha$-continuous if each restriction mapping $\varphi_{m}: \mathcal{F}_{m} \rightarrow \mathbb{C}$ is fractionally $\alpha$-bounded; that is, $\left|\varphi_{m}(b)\right| \leq$ $C_{m, \alpha}\|b\|_{m, \alpha}^{(1)}$ for all $b \in \mathcal{F}_{m}$. Its matrix version will be considered later in Subsection 7.2 .

For the detailed description of the fractional topology in the "functionally" commutative case we refer the reader to [24].

6.2. The fractional positivity. Let $M \subseteq \mathfrak{M}_{\mathcal{E}}$ be a unital subset of denominators. We introduce a positive cone in a fractional space. To have a more general scope of applications, everywhere below we fix a unital $C^{*}$-subalgebra $\mathfrak{J}_{M} \subseteq C_{\mathcal{E}}^{*}(H)$ related to $M$ (that is, it contains all $n^{-1} m, n \preceq m, n, m \in M$ ) and consider the fractional space $\mathfrak{J}_{M} / M$ instead of $C_{\mathcal{E}}^{*}(H) / M$.

Let $\mathcal{F}_{M}=\sum_{m \in M} \mathcal{F}_{m}$ be a fractional subspace in $\mathfrak{J}_{M} / M$ (see Definition [5.2). Fix an index $\alpha \in \Lambda$ and $m \in M$. Let us introduce the cone

$$
\left(\mathcal{F}_{m}\right)_{M, \alpha}^{+}=\left\{b \in \mathcal{F}_{m}: b=\sum_{n \in M, n \preceq m} b_{n}, \quad b_{n} \in \mathcal{F}_{n}, \quad b_{n} n \geq_{\alpha} 0\right\}
$$


in the subspace $\mathcal{F}_{m}$. Thus each $b \in\left(\mathcal{F}_{m}\right)_{M, \alpha}^{+}$can be written as a finite sum $b=\sum_{i=1}^{k} T_{i} / n_{i}=\left(\sum_{i=1}^{k} T_{i} n_{i}^{-1} m\right) / m$ of some $\alpha$-positive $T_{i} \in \mathfrak{J}_{M}$ such that $T_{i} / n_{i} \in \mathcal{F}_{n_{i}}, n_{i} \in M, n_{i} \preceq m, 1 \leq i \leq k$. Note that $\sum_{i=1}^{k} T_{i} n_{i}^{-1} m \in \mathfrak{J}_{M}$. We write $b \geq_{\alpha} 0$ in $\mathcal{F}_{m}$ whenever $b \in\left(\mathcal{F}_{m}\right)_{M, \alpha}^{+}$. In particular, $T / m \geq_{\alpha} 0$ in $\mathcal{F}_{m}$ whenever $T \geq_{\alpha} 0$ in $\mathfrak{J}_{M}$ and $T / m \in \mathcal{F}_{m}$. Evidently, $\left(\mathcal{F}_{m}\right)_{M, \alpha}^{+}$is a cone in $\mathcal{F}_{m}$. In particular, so is $\left(\mathfrak{J}_{M} / m\right)_{M, \alpha}^{+}$in $\mathfrak{J}_{M} / m$. We declare an element $b \in \mathcal{F}_{M}$ to be $M$-fractionally $\alpha$-positive if $b$ is a finite sum $\sum_{m} b_{m}$ of some $b_{m} \in\left(\mathcal{F}_{m}\right)_{M, \alpha}^{+}$. Put

$$
\left(\mathcal{F}_{M}\right)_{\alpha}^{+}=\sum_{m \in M}\left(\mathcal{F}_{m}\right)_{M, \alpha}^{+}
$$

which is a cone in $\mathcal{F}_{M}$ of all $M$-fractionally $\alpha$-positive elements.

Replacing the local positivity in $\mathfrak{J}_{M}$ by the global one, we obtain the cone

$$
\left(\mathcal{F}_{M}\right)^{+}=\sum_{m \in M}\left(\mathcal{F}_{m}\right)_{M}^{+}
$$

of all $M$-fractionally positive elements. In the general case, the cones $\left(\mathcal{F}_{M}\right)_{\alpha}^{+}$and $\left(\mathcal{F}_{M_{0}}\right)_{\alpha}^{+}$are different for a cofinal subset $M_{0} \subseteq M$, although $\mathcal{F}_{M}=\mathcal{F}_{M_{0}}$.

Lemma 6.6. If $n \preceq m$ for some $n, m \in M$, then $\left(\mathcal{F}_{n}\right)_{M, \alpha}^{+} \subseteq\left(\mathcal{F}_{m}\right)_{M, \alpha}^{+}$. In particular, $\left(\mathcal{F}_{M}\right)_{\alpha}^{+}=\sum_{m \in M_{0}}\left(\mathcal{F}_{m}\right)_{M, \alpha}^{+}$, where $M_{0} \subseteq M$ is a cofinal subset, and $\left(\mathcal{F}_{M}\right)_{\alpha}^{+}=\left(\mathcal{F}_{m}\right)_{M, \alpha}^{+}$whenever $m$ is the greatest element of $M$ with respect to the pre-order $\preceq$. Finally, if $N \subseteq M$, then $\left(\mathfrak{J}_{M} / N\right)_{\alpha}^{+} \subseteq\left(\mathfrak{J}_{M} / M\right)_{\alpha}^{+}$.

Proof. According to Definition [5.2, $\mathcal{F}_{n} \subseteq \mathcal{F}_{m}$ (see also Lemma 5.2) whenever $n \preceq$ $m$. Take $b \in\left(\mathcal{F}_{n}\right)_{M, \alpha}^{+}$. Then $b=\sum_{i=1}^{k} b_{i}$ with $b_{i} n_{i} \geq_{\alpha} 0, n_{i} \in M, n_{i} \preceq n$, $1 \leq i \leq k$. With $n \preceq m$ in mind, infer that $n_{i} \preceq m$ for all $i$ (we deal with the transitive relation $\preceq$ ). By its very definition, $b=\sum_{i=1}^{k} b_{i} \in\left(\mathcal{F}_{m}\right)_{M, \alpha}^{+}$.

Now let $M_{0} \subseteq M$ be a cofinal subset. For each $n \in M$ there corresponds an $m \in M_{0}$ such that $n \preceq m$. As we have just proved $\left(\mathcal{F}_{n}\right)_{M, \alpha}^{+} \subseteq\left(\mathcal{F}_{m}\right)_{M, \alpha}^{+}$, whence $\left(\mathcal{F}_{M}\right)_{\alpha}^{+}=\sum_{m \in M_{0}}\left(\mathcal{F}_{m}\right)_{M, \alpha}^{+}$.

If $M$ has the greatest element $m$, then $\mathcal{F}_{M}=\mathcal{F}_{m}$. Moreover, $\left(\mathcal{F}_{n}\right)_{M, \alpha}^{+} \subseteq$ $\left(\mathcal{F}_{m}\right)_{M, \alpha}^{+}$for all $n \in M$. Consequently, $\left(\mathcal{F}_{M}\right)_{\alpha}^{+}=\left(\mathcal{F}_{m}\right)_{M, \alpha}^{+}$.

To prove the inclusion $\left(\mathfrak{J}_{M} / N\right)_{\alpha}^{+} \subseteq\left(\mathfrak{J}_{M} / M\right)_{\alpha}^{+}$, one suffices to note that $\left(\mathfrak{J}_{M} / n\right)_{N, \alpha}^{+}$ $\subseteq\left(\mathfrak{J}_{M} / n\right)_{M, \alpha}^{+}$. Indeed, if $b \in\left(\mathfrak{J}_{M} / n\right)_{N, \alpha}^{+}$, then $b=\sum_{i=1}^{k} b_{i}$ with $b_{i} n_{i} \geq_{\alpha} 0, n_{i} \in N$, $n_{i} \preceq n, 1 \leq i \leq k$. Since $N \subseteq M$, it follows that $b \in\left(\mathfrak{J}_{M} / n\right)_{M, \alpha}^{+}$.

Remark 6.1. One should make distinct the cones $\left(\mathfrak{J}_{M} / m\right)_{M, \alpha}^{+}$and $\left(\mathfrak{J}_{M} / m\right)_{\alpha}^{+}$. Note that

$$
\left(\mathfrak{J}_{M} / m\right)_{\alpha}^{+}=\left\{T / m: T \geq_{\alpha} 0\right\} \subseteq\left(\mathfrak{J}_{M} / m\right)_{M, \alpha}^{+} .
$$

Similar cones exist over all matrix spaces too. Namely, fix $k \in \mathbb{N}$. Taking into account (6.2), infer that

$$
\mathbb{M}_{k}\left(C_{\mathcal{E}}^{*}(H) / M\right)=C_{\mathcal{E}^{k}}^{*}\left(H^{k}\right) / M^{k}=\sum_{m \in M} C_{\mathcal{E}^{k}}^{*}\left(H^{k}\right) /\left(m \cdot 1_{H^{k}}\right) .
$$


Therefore we have a cone

$$
\left(\mathbb{M}_{k}\left(C_{\mathcal{E}}^{*}(H) / M\right)\right)_{\alpha}^{+}=\sum_{m \in M}\left(C_{\mathcal{E}^{k}}^{*}\left(H^{k}\right) /\left(m \cdot 1_{H^{k}}\right)\right)_{M^{k}, \alpha}^{+}
$$

of $M^{k}$-fractionally $\alpha$-positive elements in $\mathbb{M}_{k}\left(C_{\mathcal{E}}^{*}(H) / M\right)$. By analogy,

$$
\left(\mathbb{M}_{k}\left(\mathcal{F}_{M}\right)\right)_{\alpha}^{+}=\sum_{m \in M}\left(\mathbb{M}_{k}\left(\mathcal{F}_{m}\right)\right)_{M^{k}, \alpha}^{+}
$$

is a cone of $M^{k}$-fractionally $\alpha$-positive elements in $\mathbb{M}_{k}\left(\mathcal{F}_{M}\right)$ for a fractional space $\mathcal{F}_{M}$ in $\mathfrak{J}_{M} / M$. Note that $\left(\mathbb{M}_{k}\left(\mathcal{F}_{m}\right)\right)_{M^{k}, \alpha}^{+}$cannot be thought of as $\mathbb{M}_{k}\left(\left(\mathcal{F}_{m}\right)_{M^{k}, \alpha}^{+}\right)$.

6.3. The $M$-fractionally $\alpha$-positive functionals. We say that a linear functional $\varphi: \mathcal{F}_{M} \rightarrow \mathbb{C}$ is $M$-fractionally $\alpha$-positive if it is locally positive with respect to the cone $\left(\mathcal{F}_{M}\right)_{\alpha}^{+}$(that is, $\varphi(b) \geq 0$ whenever $b \geq_{\alpha} 0$ in $\left.\mathcal{F}_{M}\right)$ and $\varphi$ is $\alpha$ compatible (that is, $\varphi(b)=0$ whenever $b={ }_{\alpha} 0$ ). In a similar manner it is defined as $M$-fractionally positive functionals. Note that $\varphi: \mathcal{F}_{M} \rightarrow \mathbb{C}$ is $M$-fractionally $\alpha$-positive iff each restriction $\varphi_{m}: \mathcal{F}_{m} \rightarrow \mathbb{C}$ is locally positive with respect to the cone $\left(\mathcal{F}_{m}\right)_{M, \alpha}^{+}, m \in M$. If $\mathcal{F}_{n} \subseteq \mathcal{F}_{m}$ for some $n, m \in M$, then $\varphi_{n}=\varphi_{m} \mid \mathcal{F}_{n}$, and $\left(\mathcal{F}_{n}\right)_{M, \alpha}^{+} \subseteq\left(\mathcal{F}_{m}\right)_{M, \alpha}^{+}$thanks to Lemma 6.6. Whence $\varphi_{m} \mid \mathcal{F}_{n}$ is positive; that is, $M$ fractionally $\alpha$-positive functionals are well defined. If $M_{0} \subseteq M$ is a cofinal subset, then $\varphi: \mathcal{F}_{M} \rightarrow \mathbb{C}$ is $M_{0}$-fractionally $\alpha$-positive if it is locally positive with respect to the cone $\left(\mathcal{F}_{M_{0}}\right)_{\alpha}^{+}$. Thus $\varphi$ is $M_{0}$-fractionally $\alpha$-positive iff each $\varphi_{m}: \mathcal{F}_{m} \rightarrow \mathbb{C}$ is positive with respect to the cone $\left(\mathcal{F}_{m}\right)_{M_{0}, \alpha}^{+}, m \in M_{0}$. Since $\left(\mathcal{F}_{m}\right)_{M_{0}, \alpha}^{+} \subseteq\left(\mathcal{F}_{m}\right)_{M, \alpha}^{+}$, $m \in M_{0}$, it follows that each $M$-fractionally $\alpha$-positive functional is automatically an $M_{0}$-fractionally $\alpha$-positive one (see also Lemma 6.6).

Proposition 6.1. Let $M_{0} \subseteq M$ be a cofinal subset. A functional $\varphi: \mathfrak{J}_{M} / M \rightarrow \mathbb{C}$ is $M_{0}$-fractionally $\alpha$-positive if and only if the mapping $\varphi$ is fractionally $\alpha$-continuous and

$$
\varphi\left(1_{H} / m\right)=\sup \left\{|\varphi(T / m)|: T \in \mathfrak{J}_{M},\|T\|_{\alpha} \leq 1\right\}
$$

for all $m \in M_{0}$.

Proof. Put $\phi_{m}: \mathfrak{J}_{M} \rightarrow \mathbb{C}, \phi_{m}(T)=\varphi_{m}(T / m)$, which is a linear functional for each $m \in M_{0}$. Note that $\phi_{m}=\varphi_{m} \theta_{m}$, where $\theta_{m}: \mathfrak{J}_{M} \rightarrow \mathfrak{J}_{M} / m$ is the isometry considered in Lemma 6.4. Note that $\varphi: \mathfrak{J}_{M} / M \rightarrow \mathbb{C}$ is fractionally $\alpha$-continuous iff all $\varphi_{m}, m \in M_{0}$, are fractionally $\alpha$-bounded (see Lemma 6.5).

Assume that $\varphi$ is fractionally $\alpha$-continuous and

$$
\varphi\left(1_{H} / m\right)=\sup \left\{|\varphi(T / m)|:\left\|T \mid H_{\alpha}\right\| \leq 1\right\}
$$

for all $m \in M_{0}$. Then for each $m \in M_{0}$ there corresponds a positive real $C_{m, \alpha}$ such that $\left|\varphi_{m}(b)\right| \leq C_{m, \alpha}\|b\|_{m, \alpha}^{(1)}, b \in \mathfrak{J}_{M} / m$. It follows that $\left|\phi_{m}(T)\right|=\left|\varphi_{m} \theta_{m}(T)\right| \leq$ $C_{m, \alpha}\left\|\theta_{m}(T)\right\|_{m, \alpha}^{(1)}=C_{m, \alpha}\|T\|_{\alpha}$ for all $T \in \mathfrak{J}_{M}$, where $\|T\|_{\alpha}=\left\|T \mid H_{\alpha}\right\|$. Thus all $\phi_{m}, m \in M_{0}$, are $\alpha$-bounded functionals. Moreover,

$$
\begin{aligned}
\phi_{m}\left(1_{H}\right) & =\varphi_{m}\left(1_{H} / m\right)=\sup \left\{|\varphi(T / m)|: T \in \mathfrak{J}_{M},\|T\|_{\alpha} \leq 1\right\} \\
& =\sup \left\{\left|\phi_{m}(T)\right|: T \in \mathfrak{J}_{M},\|T\|_{\alpha} \leq 1\right\} \\
& =\left\|\phi_{m}\right\|_{\alpha} ;
\end{aligned}
$$

that is, $\left|\phi_{m}(T)\right| \leq \phi_{m}\left(1_{H}\right)\|T\|_{\alpha}$ for all $T \in \mathfrak{J}_{M}$. Thus $\phi_{m}=\phi_{m}^{\prime} \pi_{\alpha}$ for a certain bounded functional $\phi_{m}^{\prime}: \mathfrak{J}_{\alpha} \rightarrow \mathbb{C}$, where $\pi_{\alpha}: C_{\mathcal{E}}^{*}(H) \rightarrow \mathfrak{B}_{\alpha}$ is the canonical 
projection (see Subsection [5.1), and $\mathfrak{J}_{\alpha}=\pi_{\alpha}\left(\mathfrak{J}_{M}\right)$. Furthermore, $\phi_{m}^{\prime}\left(1_{H_{\alpha}}\right)=$ $\phi_{m}\left(1_{H}\right)=\left\|\phi_{m}\right\|_{\alpha}=\left\|\phi_{m}^{\prime}\right\| . \mathfrak{J}_{\alpha}$ being a $C^{*}$-algebra, we conclude that $\phi_{m}^{\prime} \geq 0$ (see [10, 1.7.5]), or $\phi_{m} \geq_{\alpha} 0$ for all $m \in M_{0}$. Let us prove that $\varphi$ is locally positive with respect to the cone $\left(\mathfrak{J}_{M} / M_{0}\right)_{\alpha}^{+}$. Take $b \in\left(\mathfrak{J}_{M} / m\right)_{M_{0}, \alpha}^{+}, m \in M_{0}$. Then $b=\sum_{i=1}^{k} T_{i} / n_{i}$, where $T_{i} \geq_{\alpha} 0$ and $n_{i} \in M_{0}, n_{i} \preceq m$, for all $i$. Therefore

$$
\varphi_{m}(b)=\sum_{i=1}^{k} \varphi_{m}\left(T_{i} / n_{i}\right)=\sum_{i=1}^{k} \varphi_{n_{i}}\left(T_{i} / n_{i}\right)=\sum_{i=1}^{k} \phi_{n_{i}}\left(T_{i}\right) \geq 0 ;
$$

that is, $\varphi_{m}$ is $M_{0}$-fractionally $\alpha$-positive.

Conversely, if $\varphi$ is $M_{0}$-fractionally $\alpha$-positive, then $\varphi_{m}$ is locally positive with respect to the cone $\left(\mathfrak{J}_{M} / m\right)_{M_{0}, \alpha}^{+}, m \in M_{0}$. In particular, if $T \geq_{\alpha} 0$ in $\mathfrak{J}_{M}$, then $T / m \in\left(\mathfrak{J}_{M} / m\right)_{M_{0}, \alpha}^{+}$and $\varphi_{m}(T / m) \geq 0$; that is, $\phi_{m} \geq_{\alpha} 0$. Thus all $\phi_{m}$ are $\alpha$-bounded and $\left\|\phi_{m}\right\|_{\alpha}=\phi_{m}\left(1_{H}\right)$ (see [10, 1.7.3]). Hence $\varphi_{m}$ is fractionally $\alpha$ bounded and $\varphi\left(1_{H} / m\right)=\sup \left\{|\varphi(T / m)|:\|T\|_{\alpha} \leq 1\right\}$ for all $m \in M_{0}$.

\section{The INNER PRODUCT MAPPING}

In this section we introduce inner product mappings on a fractional space and investigate their properties.

Let $\Delta$ be a pre-Hilbert space with its inner product $(x, y) \mapsto\langle x, y\rangle, x, y \in \Delta$, and let $S F(\Delta)$ be a space of all sesquilinear forms on $\Delta$. Consider a unital subset $M \subseteq \mathfrak{M}_{\mathcal{E}}$ of denominators in $C_{\mathcal{E}}^{*}(H)$ and fix a $C^{*}$-subalgebra $\mathfrak{J}_{M} \subseteq C_{\mathcal{E}}^{*}(H)$ related to $M$.

Definition 7.1. Let $\mathcal{F}_{M} \subseteq \mathfrak{J}_{M} / M$ be a fractional subspace. A linear mapping $\varphi: \mathcal{F}_{M} \rightarrow S F(\Delta)$ is said to be an inner product mapping if it is unital and $\varphi\left(1_{H} / m\right)(x, x)>0, x \in \Delta \backslash\{0\}$, for all $m$ from a certain unital cofinal subset $M_{0} \subseteq M$ of positive denominators.

Thus $\varphi\left(1_{H} / 1_{H}\right)(x, y)=\langle x, y\rangle$ for all $x, y \in \Delta$. Note again that $1_{H} / 1_{H}=$ $m / m=1_{\mathcal{D}} \in \mathcal{F}_{M}$ for all $m$. Further, $\varphi\left(1_{H} / m\right)$ is an inner product on $\Delta$. Thereby $\Delta$ is a pre-Hilbert space whose completion is denoted by $\Delta_{m}$ for each $m \in M_{0}$. In particular, we have the norms $\|x\|_{m}=\left(\varphi\left(1_{H} / m\right)(x, x)\right)^{1 / 2}, m \in M_{0}$, on $\Delta$. If $\Delta=$ $\mathbb{C}$, then we deal with a unital linear functional $\varphi: \mathcal{F}_{M} \rightarrow \mathbb{C}$ such that $\varphi\left(1_{H} / m\right)>0$ for all $m \in M_{0}$. Note also that each inner-product mapping automatically involves a unital cofinal subset $M_{0} \subseteq M$ of positive denominators. Everywhere below we shall fix this cofinal subset associated by an inner-product mapping.

7.1. The quantum space $S F_{M_{0}}(\Delta)$. Let $\mathcal{F}_{M} \subseteq \mathfrak{J}_{M} / M$ be a fractional subspace and let $\varphi: \mathcal{F}_{M} \rightarrow S F(\Delta)$ be an inner product mapping. Let us introduce a subspace

$$
S F_{m}(\Delta)=\left\{\theta \in S F(\Delta):\|\theta\|_{m}<\infty\right\}
$$

where $\|\theta\|_{m}=\sup \left\{|\theta(x, y)|:\|x\|_{m} \leq 1,\|y\|_{m} \leq 1\right\}, m \in M_{0}$. We also set

$$
S F_{M_{0}}(\Delta)=\sum_{m \in M_{0}} S F_{m}(\Delta) \text {. }
$$

Evidently, each $\|\cdot\|_{m}$ is a gauge on $S F(\Delta)$. Therefore $\left(S F_{m}(\Delta),\|\cdot\|_{m}\right)$ is a normed space. Actually, the norm $\|\cdot\|_{m}$ determines a normed quantum space structure on $S F_{m}(\Delta)$. In particular, $S F_{M_{0}}(\Delta)$ turns out to be a quantum space equipped with the inductive matrix topology. Let us present some details. 
Fix $k \in \mathbb{N}$. The $k$-th Hilbert space power of $\Delta_{m}$ is denoted by $\Delta_{m}^{k}$. The relevant inner product in $\Delta_{m}^{k}$ is $\langle x, y\rangle_{m}^{(k)}=\sum_{i=1}^{k}\left\langle x_{i}, y_{i}\right\rangle_{m}$, where $x=\left[x_{i}\right], y=\left[y_{i}\right] \in \Delta_{m}^{k}$. Obviously, $\Delta_{m}^{k}$ is just the Hilbert space completion of the inner product space $\left(\Delta^{k},\langle\cdot, \cdot\rangle_{m}^{(k)}\right)$. Each matrix $\theta=\left[\theta_{i j}\right] \in \mathbb{M}_{k}(S F(\Delta))$ determines a sesquilinear form on $\Delta^{k}$ by the rule $\theta(x, y)=\sum_{i, j=1}^{k} \theta_{i j}\left(x_{j}, y_{i}\right)$, where $x=\left[x_{i}\right], y=\left[y_{i}\right] \in \Delta^{k}$. This correspondence determines the inclusion

$$
\mathbb{M}_{k}(S F(\Delta)) \subseteq S F\left(\Delta^{k}\right)
$$

up to the indicated identification.

Lemma 7.1. If $\varphi: \mathcal{F}_{M} \rightarrow S F(\Delta)$ is an inner product mapping, then so are all canonical extensions $\varphi^{(k)}: \mathbb{M}_{k}\left(\mathcal{F}_{M}\right) \rightarrow S F\left(\Delta^{k}\right)$. Moreover, $\mathbb{M}_{k}\left(S F_{M_{0}}(\Delta)\right) \subseteq$ $S F_{M_{0}^{k}}\left(\Delta^{k}\right), k \in \mathbb{N}$.

Proof. Fix $k \in \mathbb{N}$. Undoubtedly, $\varphi^{(k)}$ is unital. Note that $\mathbb{M}_{k}\left(\mathcal{F}_{M}\right) \subseteq \mathbb{M}_{k}\left(\mathfrak{J}_{M}\right) / M^{k}$ is a fractional space with the set of denominators $M^{k}=\left\{m \cdot 1_{H^{k}}: m \in M\right\}$, and $M_{0}^{k}$ is its cofinal subset (see (6.2) $)$. Moreover, $\varphi^{(k)}\left(1_{H^{k}} /\left(m \cdot 1_{H^{k}}\right)\right)(x, x)=$ $\sum_{i=1}^{k} \varphi\left(1_{H} / m\right)\left(x_{i}, x_{i}\right)>0$ whenever $x \in \Delta^{k} \backslash\{0\}$ and $m \in M_{0}$. By Definition 7.1. $\varphi^{(k)}$ is an inner product mapping.

Now take $\theta=\left[\theta_{i j}\right] \in \mathbb{M}_{k}\left(S F_{m}(\Delta)\right), m \in M_{0}$. Then

$$
\begin{aligned}
\|\theta\|_{m \cdot 1_{H^{k}}} & =\sup \left\{|\theta(x, y)|:\|x\|_{m}^{(k)} \leq 1,\|y\|_{m}^{(k)} \leq 1\right\} \\
& \leq \sup \left\{\sum_{i, j=1}^{k}\left|\theta_{i j}\left(x_{j}, y_{i}\right)\right|: \sum_{i=1}^{k}\left\|x_{i}\right\|_{m}^{2} \leq 1, \sum_{i=1}^{k}\left\|y_{i}\right\|_{m}^{2} \leq 1\right\} \\
& \leq k^{2} \max \left\{\left\|\theta_{i j}\right\|_{m}: 1 \leq i, j \leq k\right\}<\infty .
\end{aligned}
$$

Whence $\|\theta\|_{m \cdot 1_{H^{k}}}<\infty$. So, $\mathbb{M}_{k}\left(S F_{m}(\Delta)\right) \subseteq S F_{m \cdot 1_{H^{k}}}\left(\Delta^{k}\right)$. In particular,

$$
\mathbb{M}_{k}\left(S F_{M_{0}}(\Delta)\right)=\sum_{m \in M_{0}} \mathbb{M}_{k}\left(S F_{m}(\Delta)\right) \subseteq \sum_{m \in M_{0}} S F_{m \cdot 1_{H^{k}}}\left(\Delta^{k}\right)=S F_{M_{0}^{k}}\left(\Delta^{k}\right) ;
$$

that is, $\mathbb{M}_{k}\left(S F_{M_{0}}(\Delta)\right) \subseteq S F_{M_{0}^{k}}\left(\Delta^{k}\right)$.

Taking into account Lemma 7.1, we put

$$
\|\theta\|_{m}^{(k)}=\|\theta\|_{m \cdot 1_{H^{k}}} \quad \text { for } \quad \theta \in \mathbb{M}_{k}\left(S F_{m}(\Delta)\right), \quad m \in M_{0} .
$$

Thus for each $m \in M_{0}$ we have a family $s_{m}=\left\{\|\cdot\|_{m}^{(k)}: k \in \mathbb{N}\right\}$ of norms over all matrices over the space $S F_{m}(\Delta)$.

Lemma 7.2. The family $s_{m}$ is a matrix norm on $S F_{m}(\Delta)$ for each $m \in M_{0}$. In particular, $S F_{M_{0}}(\Delta)$, being an inductive limit of the normed quantum spaces $\left(S F_{m}(\Delta), s_{m}\right), m \in M_{0}$, is a quantum space (or local operator space).

Proof. Let us verify the axioms $\mathbf{M} 1$ and M2. Take $\theta \in \mathbb{M}_{k}\left(S F_{m}(\Delta)\right)$ and $\zeta \in$ $\mathbb{M}_{t}\left(S F_{m}(\Delta)\right)$. Then $\theta \oplus \zeta \in S F\left(\Delta^{k+t}\right)$ (see (7.1)), and for all $x=\left[x_{i}\right], y=\left[y_{i}\right] \in$ $\Delta^{k+t}$ we have

$$
|(\theta \oplus \zeta)(x, y)|=\left|\sum_{i, j=1}^{k} \theta_{i j}\left(x_{j}, y_{i}\right)+\sum_{i, j=k+1}^{k+t} \zeta_{i-k, j-k}\left(x_{j}, y_{i}\right)\right| \leq\left|\theta\left(x^{\prime}, y^{\prime}\right)\right|+\left|\zeta\left(x^{\prime \prime}, y^{\prime \prime}\right)\right|,
$$


where $x^{\prime}=\epsilon_{k}(x), y^{\prime}=\epsilon_{k}(y) \in \Delta^{k}$ and $x^{\prime \prime}=\epsilon^{t}(x), y^{\prime}=\epsilon^{t}(y) \in \Delta^{t}, \epsilon_{k}: \Delta^{k+t} \rightarrow$ $\Delta^{k}$ (respectively, $\epsilon^{t}: \Delta^{k+t} \rightarrow \Delta^{t}$ ) is the canonical projection onto the first $k$ (respectively, last $t$ ) coordinates. Note that

$$
\left(\|x\|_{m}^{(k+t)}\right)^{2}=\left(\left\|x^{\prime}\right\|_{m}^{(k)}\right)^{2}+\left(\left\|x^{\prime \prime}\right\|_{m}^{(t)}\right)^{2}
$$

and

It follows that

$$
\left(\|y\|_{m}^{(k+t)}\right)^{2}=\left(\left\|y^{\prime}\right\|_{m}^{(k)}\right)^{2}+\left(\left\|y^{\prime \prime}\right\|_{m}^{(t)}\right)^{2} .
$$

$$
\begin{aligned}
\|\theta \oplus \zeta\|_{m}^{(k+t)} & =\sup \left\{|(\theta \oplus \zeta)(x, y)|:\|x\|_{m}^{(k+t)},\|y\|_{m}^{(k+t)} \leq 1\right\} \\
& \leq \sup \left\{\left|\theta\left(x^{\prime}, y^{\prime}\right)\right|+\left|\zeta\left(x^{\prime \prime}, y^{\prime \prime}\right)\right|\right\} \\
& \leq \sup \left\{\|\theta\|_{m}^{(k)}\left\|x^{\prime}\right\|_{m}^{(k)}\left\|y^{\prime}\right\|_{m}^{(k)}+\|\zeta\|_{m}^{(t)}\left\|x^{\prime \prime}\right\|_{m}^{(t)}\left\|y^{\prime \prime}\right\|_{m}^{(t)}\right\} \\
& \leq \sup \left\{\max \left\{\|\theta\|_{m}^{(k)},\|\zeta\|_{m}^{(t)}\right\}\|x\|_{m}^{(k+t)}\|y\|_{m}^{(k+t)}\right\} \\
& \leq \max \left\{\|\theta\|_{m}^{(k)},\|\zeta\|_{m}^{(t)}\right\} .
\end{aligned}
$$

Thus $\|\theta \oplus \zeta\|_{m}^{(k+t)} \leq \max \left\{\|\theta\|_{m}^{(k)},\|\zeta\|_{m}^{(t)}\right\}$. Further, take $\theta \in \mathbb{M}_{k}\left(S F_{m}(\Delta)\right), \lambda \in$ $\mathbb{M}_{t, k}, \mu \in \mathbb{M}_{k, t}$. Then

$$
\begin{aligned}
|\lambda \theta \mu(x, y)| & =\left|\sum_{i, j} \sum_{p, q} \lambda_{i p} \theta_{p q} \mu_{q j}\left(x_{j}, y_{i}\right)\right| \\
& =\left|\sum_{p, q} \theta_{p q}\left(\sum_{j} \mu_{q j} x_{j}, \sum_{i} \bar{\lambda}_{i p} y_{i}\right)\right|=\left|\theta\left(\mu x, \lambda^{*} y\right)\right|
\end{aligned}
$$

and $\|\mu x\|_{m}^{(k)}=\|(\mu \otimes 1) x\|_{m}^{(k)} \leq\|\mu\|\|x\|_{m}^{(t)},\left\|\lambda^{*} y\right\|_{m}^{(k)} \leq\|\lambda\|\|y\|_{m}^{(t)}$. It follows that

$$
\|\lambda \theta \mu\|_{m}^{(t)}=\sup \left\{\left|\theta\left(\mu x, \lambda^{*} y\right)\right|:\|x\|_{m}^{(t)},\|y\|_{m}^{(t)} \leq 1\right\} \leq\|\lambda\|\|\theta\|_{m}^{(k)}\|\mu\| .
$$

Thus $s_{m}$ is a matrix norm on $S F_{m}(\Delta)$ (see [12, 2.3.6]). The rest is clear.

7.2. The matrix $\alpha$-contractive and ( $m$-fractionally) matrix $\alpha$-positive mappings. Let $\varphi: \mathcal{F}_{M} \rightarrow S F(\Delta)$ be an inner-product mapping, so we have the quantum space $S F_{M_{0}}(\Delta)$ associated by a cofinal subset $M_{0} \subseteq M$ (see Definition (7.1), and let $\varphi_{m}$ be its restriction to the subspace $\mathcal{F}_{m}$. Fix $\alpha \in \Lambda$ and let (see Lemma 6.3.

We also set

$$
\left\|\varphi_{m}^{(k)}\right\|_{m, \alpha}^{(k)}=\sup \left\{\left\|\varphi_{m}^{(k)}(b)\right\|_{m}^{(k)}:\|b\|_{m, \alpha}^{(k)} \leq 1\right\} .
$$

Note that

$$
\left\|\varphi_{m}\right\|_{m, \alpha}^{c b}=\sup \left\{\left\|\varphi_{m}^{(k)}\right\|_{m, \alpha}^{(k)}: k \in \mathbb{N}\right\}
$$

$$
\left\|\varphi_{m}\right\|_{m, \alpha}^{c b}=\sup \left\{s_{m}\left(\varphi_{m}^{(\infty)}(v)\right): q_{m, \alpha}(v) \leq 1, v \in \mathbb{M}\left(\mathcal{F}_{m}\right)\right\}
$$

(see Lemmas 7.2 and 6.3). We say that $\varphi$ is matrix $\alpha$-contractive if $\left\|\varphi_{m}\right\|_{m, \alpha}^{c b} \leq 1$ for all $m \in M_{0}$. In particular, $\varphi\left(\mathcal{F}_{m}\right) \subseteq S F_{m}(\Delta)$ for all $m \in M_{0}$, and thereupon 
$\varphi\left(\mathcal{F}_{M}\right) \subseteq S F_{M_{0}}(\Delta)$. Replacing the matrix seminorm $q_{m, \alpha}=\left\{\|\cdot\|_{m, \alpha}^{(k)}: k \in \mathbb{N}\right\}$ on $C_{\mathcal{E}}^{*}(H) / m$ (see Lemma 6.3) by the dominating matrix norm $q_{m}=\left\{\|\cdot\|_{m}^{(k)}: k \in \mathbb{N}\right\}$, we introduce a matrix contractive mapping $\varphi: \mathcal{F}_{M} \rightarrow S F(\Delta)$ with

$$
\begin{aligned}
\left\|\varphi_{m}\right\|_{m}^{c b} & =\sup \left\{\left\|\varphi_{m}^{(k)}\right\|_{m}^{(k)}: k \in \mathbb{N}\right\} \\
& =\sup \left\{s_{m}\left(\varphi_{m}^{(\infty)}(v)\right): q_{m}(v) \leq 1, v \in \mathbb{M}\left(\mathcal{F}_{m}\right)\right\} \leq 1
\end{aligned}
$$

for all $m \in M_{0}$, where $\left\|\varphi_{m}^{(k)}\right\|_{m}^{(k)}=\sup \left\{\left\|\varphi_{m}^{(k)}(b)\right\|_{m}^{(k)}:\|b\|_{m}^{(k)} \leq 1\right\}$. Thus $\varphi$ is a matrix $\alpha$-contraction if for all $m \in M_{0}, k \in \mathbb{N}, x_{1}, \ldots, x_{k}, y_{1}, \ldots, y_{k} \in \Delta$ with

$$
\sum_{i=1}^{k} \varphi\left(1_{H} / m\right)\left(x_{i}, x_{i}\right) \leq 1, \quad \sum_{i=1}^{k} \varphi\left(1_{H} / m\right)\left(y_{i}, y_{i}\right) \leq 1,
$$

and for all $b=\left[b_{i j}\right] \in \mathbb{M}_{k}\left(\mathcal{F}_{m}\right)$ with $\left\|\left[b_{i j} m\right] \mid H_{\alpha}^{k}\right\|_{\mathcal{B}\left(H_{\alpha}^{k}\right)} \leq 1$, we have

$$
\left|\sum_{i, j=1}^{k} \varphi\left(b_{i j}\right)\left(x_{j}, y_{i}\right)\right| \leq 1 .
$$

A matrix $\theta \in \mathbb{M}_{k}\left(S F_{M_{0}}(\Delta)\right)$ is said to be positive if $\theta$ is positive, being an element of $S F_{M_{0}^{k}}\left(\Delta^{k}\right)$ (see Lemma 7.1). That is,

$$
\theta(x, x) \geq 0, \quad x \in \Delta^{k} .
$$

We say that $\varphi$ is matrix $\alpha$-positive if each $\varphi^{(k)}$ is locally positive (see [9]) with respect to the cone $\mathbb{M}_{k}\left(\mathcal{F}_{M_{0}}\right)_{\alpha}^{+}$. Hence each $\varphi^{(k)}$ is $M_{0}^{k}$-fractionally $\alpha$-positive, in the sense that $\varphi_{m}^{(k)}$ is $\alpha$-compatible $\left(\varphi_{m}^{(k)}(b)=0\right.$ whenever $\left.b={ }_{\alpha} 0\right)$ and $\varphi_{m}^{(k)}(b)(x, x) \geq$ $0, x \in \Delta^{k}$, whenever $b \in\left(\mathbb{M}_{k}\left(\mathcal{F}_{m}\right)\right)_{M_{0}^{k}, \alpha}^{+}, m \in M_{0}$. If $\Delta=\mathbb{C}$, then we have a matrix $\alpha$-positive functional $\varphi: \mathcal{F}_{M} \rightarrow \mathbb{C}$ whose canonical extensions $\varphi^{(k)}: \mathbb{M}_{k}\left(\mathcal{F}_{M}\right) \rightarrow$ $\mathbb{M}_{k}$ are $M_{0}^{k}$-fractionally $\alpha$-positive, respectively.

The following assertion is a simple corollary of Proposition 6.1. To compare with the commutative case we refer the reader to [25].

Corollary 7.1. Let $\varphi: \mathfrak{J}_{M} / M \rightarrow S F(\Delta)$ be an inner-product mapping. The mapping $\varphi$ is $M_{0}$-fractionally $\alpha$-positive iff

$$
\varphi\left(1_{H} / m\right)(x, x)=\sup \left\{|\varphi(T / m)(x, x)|: T \in \mathfrak{J}_{M},\|T\|_{\alpha} \leq 1\right\}
$$

for all $m \in M_{0}, x \in \Delta$.

Proof. Fix $x \in \Delta$. The mapping $\psi_{x}: \mathfrak{J}_{M} / M \rightarrow \mathbb{C}, \psi_{x}(b)=\varphi(b)(x, x)$, is a linear functional. By its very definition, $\varphi$ is $M_{0}$-fractionally $\alpha$-positive iff all $\psi_{x}$ are $M_{0}$-fractionally $\alpha$-positive functionals (see Subsection 6.3). By Proposition 6.1. the functional $\psi_{x}$ is $M_{0}$-fractionally $\alpha$-positive if and only if $\psi_{x}\left(1_{H} / m\right)=$ $\sup \left\{\left|\psi_{x}(T / m)\right|: T \in \mathfrak{J}_{M},\|T\|_{\alpha} \leq 1\right\}$. That is,

$$
\varphi\left(1_{H} / m\right)(x, x)=\psi_{x}\left(1_{H} / m\right)=\sup \left\{|\varphi(T / m)(x, x)|: T \in \mathfrak{J}_{M},\|T\|_{\alpha} \leq 1\right\}
$$

for all $m \in M_{0}$. 
Finally, we say that a linear mapping $\Psi: \mathfrak{J}_{M} \rightarrow \mathcal{B}(K)$ is $m$-fractionally $\alpha$ positive if we have $\Psi\left(T\left(n^{-1} m\right)\right) \geq 0$ in $\mathcal{B}(K)$ for all $T \geq_{\alpha} 0$ in $\mathfrak{J}_{M}$, and $n \in M_{0}$ with $n \preceq m$. By analogy, $\Psi$ is said to be $m$-fractionally matrix $\alpha$-positive if each $\Psi^{(k)}: \mathbb{M}_{k}\left(\mathfrak{J}_{M}\right) \rightarrow \mathcal{B}\left(K^{k}\right)$ is $\left(m \cdot 1_{H^{k}}\right)$-fractionally $\alpha$-positive. That is,

$$
\Psi^{(k)}\left(T\left(n^{-1} m \cdot 1_{H^{k}}\right)\right) \geq 0
$$

in $\mathcal{B}\left(\Delta_{m}^{k}\right)$ whenever $T \in \mathbb{M}_{k}\left(\mathfrak{J}_{M}\right), T \geq_{\alpha} 0$, and $n \in M_{0}, n \preceq m$.

7.3. The quantum $\Delta$-measures. Now let us introduce the quantum $\Delta$-measures on the $C^{*}$-algebra $\mathfrak{J}_{M}$ related to a unital subset of denominators $M$ in $C_{\mathcal{E}}^{*}(H)$, where $\Delta$ is an inner product space. For a linear mapping $\mu: \mathfrak{J}_{M} / M \rightarrow S F(\Delta)$ and $x, y \in \Delta$, we use the notation $\mu_{x, y}$ to indicate the linear functional $\mu_{x, y}: \mathfrak{J}_{M} / M \rightarrow$ $\mathbb{C}, \mu_{x, y}(b)=\mu(b)(x, y)$. We also write $\mu_{x}$ instead of $\mu_{x, x}$. The polarization formula implies that

$$
4 \mu_{x, y}=\mu_{x+y}-\mu_{x-y}+i \mu_{x+i y}-i \mu_{x-i y}
$$

for all $x, y \in \Delta$.

Definition 7.2. We define a quantum $\Delta$-measure on $\mathfrak{J}_{M}$ with support in $H_{\alpha}$ if we have a unital linear mapping $\mu: \mathfrak{J}_{M} / M \rightarrow S F(\Delta)$ such that the mapping

$$
\mu_{x}^{[k]}: \mathbb{M}_{k}\left(\mathfrak{J}_{M} / M\right) \longrightarrow \mathbb{M}_{k^{2}}, \quad \mu_{x}^{[k]}(b)=\left[\begin{array}{lll}
\mu_{x_{1}, x_{1}}^{(k)}(b) & \cdots & \mu_{x_{k}, x_{1}}^{(k)}(b) \\
\vdots & & \vdots \\
\mu_{x_{1}, x_{k}}^{(k)}(b) & \cdots & \mu_{x_{k}, x_{k}}^{(k)}(b)
\end{array}\right]
$$

is $M_{0}^{k}$-fractionally $\alpha$-positive for each $x=\left[x_{i}\right] \in \Delta^{k}, k \in \mathbb{N}$, and for a certain unital cofinal subset $M_{0} \subseteq M$ of positive denominators.

By analogy, replacing the local $\alpha$-positivity by the "global" one, it is defined as in Definition 7.2 as the quantum $\Delta$-measure on $\mathfrak{J}_{M}$ (with support in $\mathcal{D}$ ).

Lemma 7.3. If $\mu$ is a quantum $\Delta$-measure on $\mathfrak{J}_{M}$ with support in $H_{\alpha}$, then each functional $\mu_{x}: \mathfrak{J}_{M} / M \rightarrow \mathbb{C}$ is matrix $\alpha$-positive.

Proof. Indeed, by Definition [7.2, $\mu_{x}^{[1]}: \mathfrak{J}_{M} / M \rightarrow \mathbb{C}$ is $M_{0}$-fractionally $\alpha$-positive for each $x \in \Delta$. But $\mu_{x}^{[1]}(b)=\mu_{x, x}^{(1)}(b)=\mu_{x}(b)$ for all $b \in \mathfrak{J}_{M} / M$; that is, $\mu_{x}$ is $M_{0}$-fractionally $\alpha$-positive for all $x \in \Delta$. Moreover, if $y=[x, 0, \ldots, 0] \in \Delta^{k}$ and $b=\left[b_{i j}\right] \in\left(\mathbb{M}_{k}\left(\mathfrak{J}_{M}\right) / M_{0}^{k}\right)_{\alpha}^{+}$, then

$$
\mu_{y}^{[k]}(b)=\left[\begin{array}{lll}
\mu_{x}^{(k)}(b) & \cdots & 0 \\
\vdots & \ddots & \vdots \\
0 & \cdots & 0
\end{array}\right] \geq 0
$$

thanks to Definition 7.2. Whence $\mu_{x}^{(k)}(b) \geq 0$. Thus $\mu_{x}^{(k)}: \mathbb{M}_{k}\left(\mathfrak{J}_{M}\right) / M^{k} \rightarrow \mathbb{M}_{k}$ is $M_{0}^{k}$-fractionally $\alpha$-positive, which means that $\mu_{x}: \mathfrak{J}_{M} / M \rightarrow \mathbb{C}$ is matrix $\alpha$-positive (see Subsection 7.2).

Lemma 7.4. If $\mu$ is a quantum $\Delta$-measure on $\mathfrak{J}_{M}$ with support in $H_{\alpha}$, then the relevant linear mapping $\mu: \mathfrak{J}_{M} / M \rightarrow S F(\Delta)$ is an inner product mapping. 
Proof. By Definition 7.2, $\mu$ involves a unital cofinal subset $M_{0} \subseteq M$. Take $m \in$ $M_{0}$. Since $m \geq 0$, it follows that $\left\|m \mid H_{\alpha}\right\| 1_{H} \geq_{\alpha} m$ or $1_{H}-\|m\|_{\alpha}^{-1} m \geq_{\alpha} 0$. But $1_{H}-\|m\|_{\alpha}^{-1} m \in \mathfrak{J}_{M}$ and $\left(1_{H}-\|m\|_{\alpha}^{-1} m\right) / m \in\left(\mathfrak{J}_{M} / m\right)_{M_{0}, \alpha}^{+}$. Moreover, $\|m\|_{\alpha}^{-1} m / m=\|m\|_{\alpha}^{-1} 1_{H} / 1_{H}=\|m\|_{\alpha}^{-1} 1_{\mathcal{D}}$. Fix $x \in \Delta \backslash\{0\}$. Taking into account that $\mu_{x}: \mathfrak{J}_{M} / M \rightarrow \mathbb{C}$ is $M_{0}$-fractionally $\alpha$-positive (see Lemma 7.3) and $1_{H} / m \in$ $\left(\mathfrak{J}_{M} / m\right)_{M_{0}, \alpha}^{+}$, we deduce that

$\mu\left(1_{H} / m\right)(x, x)=\mu_{x}\left(1_{H} / m\right) \geq\|m\|_{\alpha}^{-1} \mu_{x}\left(1_{\mathcal{D}}\right)=\|m\|_{\alpha}^{-1}\langle x, x\rangle=\|m\|_{\alpha}^{-1}\|x\|^{2}>0$.

By Definition 7.1 $\mu$ is an inner product mapping with the same cofinal subset $M_{0}$.

Theorem 7.1. The linear mapping $\mu: \mathfrak{J}_{M} / M \rightarrow S F(\Delta)$ is a quantum $\Delta$-measure on $\mathfrak{J}_{M}$ with support in $H_{\alpha}$ if and only if $\mu$ is an inner product mapping, which is matrix $\alpha$-positive. Moreover, in this case, $\mu\left(\mathfrak{J}_{M} / m\right) \subseteq S F_{m}(\Delta), m \in M_{0}$, for a certain cofinal subset $M_{0} \subseteq M$.

Proof. Assume that $\mu$ is a quantum $\Delta$-measure on $\mathfrak{J}_{M}$ with support in $H_{\alpha}$. By Lemma 7.4, $\mu: \mathfrak{J}_{M} / M \rightarrow S F(\Delta)$ is an inner product mapping with the same cofinal subset $M_{0}$. Take $b=\left[b_{i j}\right] \in\left(\mathbb{M}_{k}\left(\mathfrak{J}_{M}\right) / M_{0}^{k}\right)_{\alpha}^{+}$, and fix $x=\left[x_{i}\right] \in \Delta^{k}$. We set $\epsilon_{1}, \ldots, \epsilon_{k}$ for the canonical basis in $\mathbb{C}^{k}$, and $\epsilon=\left[\epsilon_{i}\right]$ is the (column) vector in $\mathbb{C}^{k^{2}}$. Then

$$
\begin{aligned}
\mu^{(k)}(b)(x, x) & =\left[\mu\left(b_{i j}\right)\right](x, x)=\sum_{i, j=1}^{k} \mu\left(b_{i j}\right)\left(x_{j}, x_{i}\right)=\sum_{i, j=1}^{k} \mu_{x_{j}, x_{i}}\left(b_{i j}\right) \\
& =\sum_{i, j}\left\langle\mu_{x_{j}, x_{i}}^{(k)}(b) \epsilon_{j}, \epsilon_{i}\right\rangle=\sum_{i}\left\langle\sum_{j} \mu_{x_{j}, x_{i}}^{(k)}(b) \epsilon_{j}, \epsilon_{i}\right\rangle \\
& =\left\langle\left[\begin{array}{lll}
\mu_{x_{1}, x_{1}}^{(k)}(b) & \cdots & \mu_{x_{k}, x_{1}}^{(k)}(b) \\
\vdots & & \vdots \\
\mu_{x_{1}, x_{k}}^{(k)}(b) & \cdots & \mu_{x_{k}, x_{k}}^{(k)}(b)
\end{array}\right]\left[\begin{array}{l}
\epsilon_{1} \\
\vdots \\
\epsilon_{k}
\end{array}\right],\left[\begin{array}{l}
\epsilon_{1} \\
\vdots \\
\epsilon_{k}
\end{array}\right]\right\rangle \\
& =\left\langle\mu_{x}^{[k]}(b) \epsilon, \epsilon\right\rangle .
\end{aligned}
$$

By Definition $7.2, \mu_{x}^{[k]}(b) \geq 0$. Therefore $\left\langle\mu_{x}^{[k]}(b) \epsilon, \epsilon\right\rangle \geq 0$. It follows that $\mu^{(k)}(b)(x, x) \geq 0$; that is, $\mu^{(k)}(b) \geq 0$. Whence $\mu^{(k)}$ is $M_{0}^{k}$-fractionally $\alpha$-positive (see Subsection [7.2). Using Corollary 7.1, infer that

$$
\sup \left\{\left|\mu_{x}(T / m)\right|: T \in \mathfrak{J}_{M},\|T\|_{\alpha} \leq 1\right\}=\mu_{x}\left(1_{H} / m\right)=\|x\|_{m}^{2}
$$

for all $m \in M_{0}, x \in \Delta$. Further, taking into account (7.2) and using the Parallelogram Law (see for instance [17, 6.1.8]), we obtain that

$$
\begin{aligned}
4|\mu(T / m)(x, y)| & =4\left|\mu_{x, y}(T / m)\right| \leq\|x+y\|_{m}^{2}+\|x-y\|_{m}^{2}+\|x+i y\|_{m}^{2}+\|x-i y\|_{m}^{2} \\
& =2\left(\|x\|_{m}^{2}+\|y\|_{m}^{2}\right)+2\left(\|x\|_{m}^{2}+\|y\|_{m}^{2}\right)=4\left(\|x\|_{m}^{2}+\|y\|_{m}^{2}\right)
\end{aligned}
$$

whenever $\|T\|_{\alpha} \leq 1$. It follows that $\|\mu(T / m)\|_{m} \leq 2$; that is, $\mu(T / m) \in S F_{m}(\Delta)$. Thus $\mu\left(\mathfrak{J}_{M} / m\right) \subseteq S F_{m}(\Delta)$ for all $m \in M_{0}$. In particular, $\mu\left(\mathfrak{J}_{M} / M\right) \subseteq S F_{M_{0}}(\Delta)$. 
Now assume that $\mu$ is a matrix $\alpha$-positive inner product mapping. Fix $x=\left[x_{i}\right] \in$ $\Delta^{k}$ and $b=\left[b_{t s}\right] \in\left(\mathbb{M}_{k}\left(\mathfrak{J}_{M}\right) / M_{0}^{k}\right)_{\alpha}^{+}$. Let us prove that $\mu_{x}^{[k]}(b) \geq 0$ in $\mathbb{M}_{k^{2}}$ (see Definition 7.2). Take $\xi=\left[\xi_{i}\right] \in \mathbb{C}^{k^{2}}$, where each $\xi_{i}=\left[\xi_{i j}\right] \in \mathbb{C}^{k}$ is the (column) vector. Put also $\widehat{\xi}=\left[\xi_{i j}\right] \in \mathbb{M}_{k}$. Then

$$
\begin{aligned}
\left\langle\mu_{x}^{[k]}(b) \xi, \xi\right\rangle & =\left\langle\left[\begin{array}{lll}
\mu_{x_{1}, x_{1}}^{(k)}(b) & \cdots & \mu_{x_{k}, x_{1}}^{(k)}(b) \\
\vdots & & \vdots \\
\mu_{x_{1}, x_{k}}^{(k)}(b) & \cdots & \mu_{x_{k}, x_{k}}^{(k)}(b)
\end{array}\right]\left[\begin{array}{l}
\xi_{1} \\
\vdots \\
\xi_{k}
\end{array}\right],\left[\begin{array}{l}
\xi_{1} \\
\vdots \\
\xi_{k}
\end{array}\right]\right\rangle \\
& =\sum_{j}\left\langle\left[\begin{array}{l}
\mu_{x_{j}, x_{1}}^{(k)}(b) \xi_{j} \\
\vdots \\
\mu_{x_{j}, x_{k}}^{(k)}(b) \xi_{j}
\end{array}\right],\left[\begin{array}{l}
\xi_{1} \\
\vdots \\
\xi_{k}
\end{array}\right]\right\rangle=\sum_{j, i}\left\langle\mu_{x_{j}, x_{i}}^{(k)}(b) \xi_{j}, \xi_{i}\right\rangle \\
& =\sum_{j, i}\left\langle\left[\begin{array}{l}
\sum_{s} \mu_{x_{j}, x_{i}}\left(b_{1 s}\right) \xi_{j s} \\
\vdots \\
\sum_{s} \mu_{x_{j}, x_{i}}\left(b_{k s}\right) \xi_{j s}
\end{array}\right],\left[\begin{array}{l}
\xi_{i 1} \\
\vdots \\
\xi_{i k}
\end{array}\right]\right\rangle=\sum_{j, i, s, t} \xi_{j s} \mu_{x_{j}, x_{i}}\left(b_{t s}\right) \bar{\xi}_{i t} \\
& =\sum_{j, i, s, t} \xi_{j s} \mu\left(b_{t s}\right)\left(x_{j}, x_{i}\right) \bar{\xi}_{i t}=\sum_{s, t} \mu\left(b_{t s}\right)\left(\sum_{j} \xi_{j s} x_{j}, \sum_{i} \xi_{i t} x_{i}\right) \\
& =\mu^{(k)}(b)\left(\widehat{\xi} x, \widehat{\xi}_{x}\right) \geq 0,
\end{aligned}
$$

where $\mu$ is matrix $\alpha$-positive. Thus $\mu$ is a quantum $\Delta$-measure on $\mathfrak{J}_{M}$ with support in $H_{\alpha}$.

7.4. The quantum $\mathcal{B}(K)$-valued measures. Now again let $\Delta$ be an inner product space whose completion is $K$ and let $\Psi: \mathfrak{J}_{M} \rightarrow \mathcal{B}(K)$ be a linear mapping. The mapping $\Psi$ can be extended up to a linear mapping $\mu: \mathfrak{J}_{M} / M \rightarrow S F(\Delta)$ by the following canonical method. A few elements $x, y \in \Delta$ determines the linear functional

$$
\mu_{x, y}: \mathfrak{J}_{M} \longrightarrow \mathbb{C}, \quad \mu_{x, y}(T)=\langle\Psi(T) x, y\rangle .
$$

Assume they admit linear extensions $\widetilde{\mu}_{x, y}: \mathfrak{J}_{M} / M \rightarrow \mathbb{C}$ such that the mapping $\Delta \times \Delta \rightarrow \mathbb{C},(x, y) \mapsto \widetilde{\mu}_{x, y}(b)$, is a sesquilinear form on $\Delta$ for each $b \in \mathfrak{J}_{M} / M$. Hence we have a linear mapping

$$
\mu: \mathfrak{J}_{M} / M \longrightarrow S F(\Delta), \quad \mu(b)(x, y)=\widetilde{\mu}_{x, y}(b) .
$$

In this case, we say that $\mu: \mathfrak{J}_{M} / M \rightarrow S F(\Delta)$ is a sesquilinear extension of the linear mapping $\Psi: \mathfrak{J}_{M} \rightarrow \mathcal{B}(K)$.

Definition 7.3. A unital linear mapping $\Psi: \mathfrak{J}_{M} \rightarrow \mathcal{B}(K)$ is said to be a quantum $\mathcal{B}(K)$-valued measure on $\mathfrak{J}_{M}$ with support in $H_{\alpha}$ if it has a sesquilinear extension $\mu: \mathfrak{J}_{M} / M \rightarrow S F(\Delta)$ which is a quantum $\Delta$-measure on $\mathfrak{J}_{M}$ with support in $H_{\alpha}$.

Note that a quantum $\mathcal{B}(K)$-valued measure $\Psi: \mathfrak{J}_{M} \rightarrow \mathcal{B}(K)$ is a matrix $\alpha$ positive mapping automatically. Indeed, take $b \in \mathbb{M}_{k}\left(\mathfrak{J}_{M}\right)$ such that $b \geq_{\alpha} 0$ and 
take $x \in \Delta^{k}$. Then

$$
\begin{aligned}
\left\langle\Psi^{(k)}(b) x, x\right\rangle & =\sum_{i, j}\left\langle\Psi\left(b_{i j}\right) x_{j}, x_{i}\right\rangle=\sum_{i, j} \mu_{x_{j}, x_{i}}\left(b_{i j}\right) \\
& =\sum_{i, j} \widetilde{\mu}_{x_{j}, x_{i}}\left(b_{i j}\right)=\sum_{i, j} \mu\left(b_{i j}\right)\left(x_{j}, x_{i}\right) \\
& =\mu^{(k)}(b)(x, x) \geq 0
\end{aligned}
$$

for $b=b / 1_{\mathcal{D}^{k}} \in\left(\mathbb{M}_{k}\left(\mathfrak{J}_{M}\right) / 1_{\mathcal{D}^{k}}\right)_{\alpha}^{+} \subseteq\left(\mathbb{M}_{k}\left(\mathfrak{J}_{M}\right) / 1_{\mathcal{D}^{k}}\right)_{M_{0}^{k}, \alpha}^{+}$(see Remark 6.1).

The following assertion describes the quantum scalar measures.

Proposition 7.1. Assume that $\Delta=K=\mathbb{C}$ and that $\Psi: \mathfrak{J}_{M} \rightarrow \mathbb{C}$ is a unital linear functional. Then $\Psi$ determines a quantum $\mathbb{C}$-valued measure on $\mathfrak{J}_{M}$ with support in $H_{\alpha}$ if and only if $\Psi$ extends up to a matrix $\alpha$-positive functional $\widetilde{\Psi}: \mathfrak{J}_{M} / M \rightarrow \mathbb{C}$ such that $\widetilde{\Psi}\left(1_{H} / m\right)>0$ for all $m \in M_{0}$, where $M_{0} \subseteq M$ is a cofinal subset. In particular,

$$
\left\|\widetilde{\Psi}_{m}\right\|_{m, \alpha}^{c b}=\widetilde{\Psi}\left(1_{H} / m\right) \quad \text { for all } m \in M_{0} .
$$

Proof. First, assume that $\Psi$ determines a quantum $\mathbb{C}$-measure $\mu: \mathfrak{J}_{M} / M \rightarrow S F(\mathbb{C})$ on $\mathfrak{J}_{M}$ with support in $H_{\alpha}$. Using Lemma 7.3 and Definition 7.3, we conclude that each $\mu_{x}: \mathfrak{J}_{M} \rightarrow \mathbb{C}, \mu_{x}(T)=|x|^{2} \Psi(T), x \in \mathbb{C}$, has a matrix $\alpha$-positive extension $\widetilde{\mu}_{x}: \mathfrak{J}_{M} / M \rightarrow \mathbb{C}$. In particular, $\Psi=\mu_{1}(1$ is the unit in $\mathbb{C})$ extends up to a matrix $\alpha$-positive mapping $\widetilde{\mu}_{1}$. It remains to set $\widetilde{\Psi}=\widetilde{\mu}_{1}$. Note that $\widetilde{\Psi}\left(1_{H} / m\right)=\widetilde{\mu}_{1}\left(1_{H} / m\right)=\mu\left(1_{H} / m\right)>0$ for all $m \in M_{0}$.

Now assume that $\Psi$ extends up to a matrix $\alpha$-positive functional $\widetilde{\Psi}: \mathfrak{J}_{M} / M \rightarrow \mathbb{C}$ such that $\widetilde{\Psi}\left(1_{H} / m\right)>0$ for all $m \in M_{0}$. So, all extensions $\widetilde{\Psi}^{(k)}: \mathbb{M}_{k}\left(\mathfrak{J}_{M} / M\right) \rightarrow$ $\mathbb{M}_{k}$ are $M_{0}^{k}$-fractionally $\alpha$-positive. Then all functionals $\mu_{x, y}: \mathfrak{J}_{M} \rightarrow \mathbb{C}, \mu_{x, y}(T)=$ $x \Psi(T) \bar{y}, x, y \in \mathbb{C}$, have linear extensions $\widetilde{\mu}_{x, y}: \mathfrak{J}_{M} / M \rightarrow \mathbb{C}, \widetilde{\mu}_{x, y}(b)=x \widetilde{\Psi}(b) \bar{y}$. Evidently, the mapping $\mathbb{C} \times \mathbb{C} \rightarrow \mathbb{C},(x, y) \mapsto \widetilde{\mu}_{x, y}(b)$ is a sesquilinear form for each $b \in \mathfrak{J}_{M} / M$, and we have a well defined linear mapping

$$
\mu: \mathfrak{J}_{M} / M \longrightarrow S F(\mathbb{C}), \quad \mu(b)(x, y)=\widetilde{\mu}_{x, y}(b) .
$$

Note that $\mu\left(1_{H} / m\right)(x, x)=\widetilde{\mu}_{x}\left(1_{H} / m\right)=|x|^{2} \widetilde{\Psi}\left(1_{H} / m\right)>0$ if $x \in \mathbb{C} \backslash\{0\}$. It remains to prove that $\mu$ is a quantum $\Delta$-measure with support in $H_{\alpha}$; that is, $\mu_{x}^{[k]}(b) \geq 0$ (see Definition 7.2) whenever $x=\left[x_{i}\right] \in \mathbb{C}^{k}$ and $b \in\left(\mathbb{M}_{k}\left(\mathfrak{J}_{M}\right) / M_{0}^{k}\right)_{\alpha}^{+}$. Note that $\widetilde{\mu}_{x, y}^{(k)}(b)=x \widetilde{\Psi}^{(k)}(b) \bar{y}$ for all $x, y \in \mathbb{C}$. It follows that

$$
\begin{aligned}
\mu_{x}^{[k]}(b)= & {\left[\begin{array}{lll}
x_{1} \widetilde{\Psi}^{(k)}(b) \bar{x}_{1} & \cdots & x_{k} \widetilde{\Psi}^{(k)}(b) \bar{x}_{1} \\
\vdots & & \vdots \\
x_{1} \widetilde{\Psi}^{(k)}(b) \bar{x}_{k} & \cdots & x_{k} \widetilde{\Psi}^{(k)}(b) \bar{x}_{k}
\end{array}\right] } \\
= & {\left[\begin{array}{l}
\bar{x}_{1} I_{k} \\
\vdots \\
\bar{x}_{k} I_{k}
\end{array}\right] \widetilde{\Psi}^{(k)}(b)\left[\begin{array}{lll}
x_{1} I_{k} & \cdots & x_{k} I_{k}
\end{array}\right] . }
\end{aligned}
$$

By assumption, $\widetilde{\Psi}$ is matrix $\alpha$-positive, therefore $\widetilde{\Psi}^{(k)}(b) \geq 0$ in $\mathbb{M}_{k}$. It follows that $\mu_{x}^{[k]}(b) \geq 0$ in $\mathbb{M}_{k^{2}}$. Thus $\mu$ is a quantum $\Delta$-measure. 
Finally, note that $\left\|\widetilde{\Psi}_{m}\right\|_{m, \alpha}=\sup \left\{|\widetilde{\Psi}(T / m)|: T \in \mathfrak{J}_{M},\|T\|_{\alpha} \leq 1\right\}=\widetilde{\Psi}\left(1_{H} / m\right)$ thanks to Proposition 6.1. Using Lemma 6.4 and [13, Lemma 5.2] (see also [12, 2.2.3]), we derive that $\left\|\widetilde{\Psi}_{m}\right\|_{m, \alpha}^{c b}=\left\|\widetilde{\Psi}_{m}\right\|_{m, \alpha}=\widetilde{\Psi}\left(1_{H} / m\right)$ for all $m \in M_{0}$.

Thus unital matrix positive functionals $\mu: \mathfrak{J}_{M} / M \rightarrow \mathbb{C}$ determine quantum scalar measures on the $C^{*}$-algebra $\mathfrak{J}_{M}$.

The following lemma is very similar to one in [2, Lemma 2.4] proved for the commutative case. For convenience, we present all details.

Lemma 7.5. Let $\psi: \mathfrak{J}_{M} / M \rightarrow S F(\Delta)$ be an inner-product mapping, which determines a family $\Delta_{m}, m \in M_{0}$, of Hilbert space completions of $\Delta$. If $\psi_{m}$ is $M_{0}$-fractionally $\alpha$-positive, then

$$
\psi(T / m)(x, y)=\left\langle\Psi_{m}(T) x, y\right\rangle_{m}, \quad T \in \mathfrak{J}_{M}, \quad x, y \in \Delta,
$$

for the uniquely determined unital $\alpha$-positive linear mapping $\Psi_{m}: \mathfrak{J}_{M} \rightarrow \mathcal{B}\left(\Delta_{m}\right)$, $m \in M_{0}$. In this case, $\psi_{m}$ is matrix $\alpha$-contractive iff $\Psi_{m}$ is as well. Further, $\psi_{m}$ is matrix $\alpha$-positive iff $\Psi_{m}$ is m-fractionally matrix $\alpha$-positive on $\mathfrak{J}_{M}$. Finally, if $n \preceq m$ for some $n, m \in M_{0}$, then there is a unique $\alpha$-positive mapping $\Psi_{m, n}$ : $\mathfrak{J}_{M} \rightarrow \mathcal{B}\left(\Delta_{n}\right)$ such that

$$
\left\langle\Psi_{m, n}(T) x, y\right\rangle_{n}=\psi(T / n)(x, y)=\left\langle\Psi_{m}\left(T n^{-1} m\right) x, y\right\rangle_{m}
$$

for all $x, y \in \Delta$. In this case, $\Psi_{m, n}$ is matrix $\alpha$-contractive iff $\psi_{n}$ is as well. Moreover, $\Psi_{m, n}$ is n-fractionally matrix $\alpha$-positive iff $\psi_{n}$ is matrix $\alpha$-positive.

Proof. Take $T \in \mathfrak{J}_{M}$ such that $T \geq_{\alpha} 0$. Then $\|T\|_{\alpha} 1_{H} / m-T / m \geq_{\alpha} 0$ in $\mathfrak{J}_{M} / m$ for $\|T\|_{\alpha} 1_{H} \geq_{\alpha} T$; that is, $\|T\|_{\alpha} 1_{H} / m-T / m \in\left(\mathfrak{J}_{M} / m\right)_{M_{0}, \alpha}^{+}$. Hence $\|T\|_{\alpha} \psi_{m}\left(1_{H} / m\right)(x, x)-\psi_{m}(T / m)(x, x) \geq 0$; that is, $\psi_{m}(T / m)(x, x) \leq$ $\|T\|_{\alpha}\langle x, x\rangle_{m}$ for all $x \in \Delta$. Note that $\psi_{m}(T / m)(x, y), x, y \in \Delta$, is a positive semi-definite form on $\Delta$. Using the Cauchy-Schwarz inequality [17, 6.1.5], we infer that

$$
\left|\psi_{m}(T / m)(x, y)\right| \leq\|T\|_{\alpha}\|x\|_{m}\|y\|_{m}
$$

for all $x, y \in \Delta$. Using the density of $\Delta$ in $\Delta_{m}$, we conclude that the latter inequality holds for all $x, y \in \Delta_{m}$. Whence $\psi_{m}(T / m)(x, y)=\left\langle\Psi_{m}^{\prime}\left(T \mid H_{\alpha}\right) x, y\right\rangle_{m}$ for a positive operator $\Psi_{m}^{\prime}\left(T \mid H_{\alpha}\right) \in \mathcal{B}\left(\Delta_{m}\right)$ (see for instance [17, 6.5.3]). Moreover, $\Psi_{m}^{\prime}\left(T\left|H_{\alpha}+S\right| H_{\alpha}\right)=\Psi_{m}^{\prime}\left(T \mid H_{\alpha}\right)+\Psi_{m}^{\prime}\left(S \mid H_{\alpha}\right)$ and $\Psi_{m}^{\prime}\left(r T \mid H_{\alpha}\right)=r \Psi_{m}^{\prime}\left(T \mid H_{\alpha}\right)$ for all $\alpha$-positive $T, S$ and a positive $r \in \mathbb{R}$. Let us remark that each element of the $C^{*}$-algebra $\mathfrak{J}_{\alpha}=\pi_{\alpha}\left(\mathfrak{J}_{M}\right)$ is a linear combination of four positive operators from $\mathfrak{J}_{\alpha}$. Therefore $\psi_{m}(T / m)(x, y)=\left\langle\Psi_{m}^{\prime}\left(T \mid H_{\alpha}\right) x, y\right\rangle_{m}$ for a positive linear mapping $\Psi_{m}^{\prime}: \mathfrak{J}_{\alpha} \rightarrow \mathcal{B}\left(\Delta_{m}\right)$. It remains to set $\Psi_{m}=\Psi_{m}^{\prime} \pi_{\alpha}$. Note also that $\left\langle\Psi_{m}\left(1_{H}\right) x, y\right\rangle_{m}=\psi_{m}\left(1_{H} / m\right)(x, y)=\langle x, y\rangle_{m}$; that is, $\Psi_{m}\left(1_{H}\right)=1_{\Delta_{m}}$. Hence $\Psi_{m}: \mathfrak{J}_{M} \rightarrow \mathcal{B}\left(\Delta_{m}\right)$ is a unital $\alpha$-positive mapping.

As we have indicated above (see (6.2) $), \mathbb{M}_{k}\left(\mathfrak{J}_{M} / m\right) \subseteq C_{\mathcal{E}^{k}}^{*}\left(H^{k}\right) /\left(m \cdot 1_{H^{k}}\right)$, $k \in \mathbb{N}$. Moreover, $\psi_{m}^{(k)}\left(T /\left(m \cdot 1_{H^{k}}\right)\right)(x, y)=\sum_{i, j=1}^{k} \psi_{m}\left(T_{i j} / m\right)\left(x_{j}, y_{i}\right)$ for all $T=\left[T_{i j}\right] \in \mathbb{M}_{k}\left(\mathfrak{J}_{M}\right), x=\left[x_{i}\right], y=\left[y_{i}\right] \in \Delta^{k}$. It follows that

$$
\psi_{m}^{(k)}\left(T /\left(m \cdot 1_{H^{k}}\right)\right)(x, y)=\sum_{i, j=1}^{k}\left\langle\Psi_{m}\left(T_{i j}\right) x_{j}, y_{i}\right\rangle_{m}=\left\langle\Psi_{m}^{(k)}(T) x, y\right\rangle_{m}^{(k)},
$$


where $\Psi_{m}^{(k)}: \mathbb{M}_{k}\left(\mathfrak{J}_{M}\right) \rightarrow \mathcal{B}\left(\Delta_{m}^{k}\right)$ is the canonical extension of $\Psi_{m}$. Further,

$$
\begin{aligned}
& \| \psi_{m}^{(k)}\left(T /\left(m \cdot 1_{H^{k}}\right)\right) \|_{m}^{(k)} \\
&= \sup \left\{\left|\psi_{m}^{(k)}\left(T /\left(m \cdot 1_{H^{k}}\right)\right)(x, y)\right|:\|x\|_{m}^{(k)} \leq 1,\|y\|_{m}^{(k)} \leq 1\right\} \\
& \quad=\sup \left\{\left|\left\langle\Psi_{m}^{(k)}(T) x, y\right\rangle_{m}^{(k)}\right|: x, y \in \Delta_{m}^{k},\|x\|_{m}^{(k)} \leq 1,\|y\|_{m}^{(k)} \leq 1\right\} \\
&=\left\|\Psi_{m}^{(k)}(T)\right\|_{\mathcal{B}\left(\Delta_{m}^{k}\right)} .
\end{aligned}
$$

It follows that

$$
\left\|\psi_{m}^{(k)}\right\|_{m, \alpha}^{(k)}=\sup \left\{\left\|\psi_{m}^{(k)}\left(T /\left(m \cdot 1_{H^{k}}\right)\right)\right\|_{m}^{(k)}:\left\|T \mid H_{\alpha}^{k}\right\| \leq 1\right\}=\left\|\Psi_{m}^{(k)}\right\|_{\alpha}^{(k)} .
$$

In particular, $\left\|\psi_{m}\right\|_{m, \alpha}^{c b}=\sup \left\{\left\|\psi_{m}^{(k)}\right\|_{m, \alpha}^{(k)}: k \in \mathbb{N}\right\}=\sup \left\{\left\|\Psi_{m}^{(k)}\right\|_{\alpha}^{(k)}: k \in \mathbb{N}\right\}=$ $\left\|\Psi_{m}\right\|_{\alpha}^{c b}$. Thereby, $\psi_{m}$ is matrix $\alpha$-contractive iff $\Psi_{m}$ is as well.

Now take an $M_{0}^{k}$-fractionally $\alpha$-positive element $b$ in $\mathbb{M}_{k}\left(\mathfrak{J}_{M}\right) /\left(m \cdot 1_{H^{k}}\right)$; that is,

$$
b \in\left(\mathbb{M}_{k}\left(\mathfrak{J}_{M}\right) /\left(m \cdot 1_{H^{k}}\right)\right)_{M_{0}^{k}, \alpha}^{+} .
$$

Then $b=\sum_{i=1}^{t} T_{i} /\left(n_{i} \cdot 1_{H^{k}}\right)$ for some $n_{i} \in M_{0}, n_{i} \preceq m$, and $T_{i} \geq_{\alpha} 0$ in $C_{\mathcal{E}^{k}}^{*}\left(H^{k}\right)$, $1 \leq i \leq t$. It follows that

$$
\begin{aligned}
\psi_{m}^{(k)}(b)(x, x) & =\sum_{i=1}^{t} \psi_{m}^{(k)}\left(T_{i} /\left(n_{i} \cdot 1_{H^{k}}\right)\right)(x, x) \\
& =\sum_{i=1}^{t} \psi_{m}^{(k)}\left(T_{i}\left(n_{i}^{-1} m \cdot 1_{H^{k}}\right) /\left(m \cdot 1_{H^{k}}\right)\right)(x, x) \\
& =\sum_{i=1}^{t}\left\langle\Psi_{m}^{(k)}\left(T_{i}\left(n_{i}^{-1} m \cdot 1_{H^{k}}\right)\right) x, x\right\rangle_{m}^{(k)} \\
& =\left\langle\Psi_{m}^{(k)}\left(\sum_{i=1}^{t} T_{i}\left(n_{i}^{-1} m \cdot 1_{H^{k}}\right)\right) x, x\right\rangle_{m}^{(k)}
\end{aligned}
$$

for all $k \in \mathbb{N}$. Using the density of $\Delta$ in $\Delta_{m}$, we derive that $\Psi_{m}^{(k)}$ is $m$-fractionally $\alpha$-positive (see Subsection 7.2) iff $\psi_{m}^{(k)}$ is $M_{0}^{k}$-fractionally $\alpha$-positive (that is, with respect to the cone $\left.\left(\mathbb{M}_{k}\left(\mathfrak{J}_{M}\right) / m\right)_{M_{0}^{k}, \alpha}^{+}\right)$. Whence $\Psi_{m}$ is $m$-fractionally matrix $\alpha$ positive iff $\psi_{m}$ is matrix $\alpha$-positive.

Now let $n \preceq m$ for some $n \in M_{0}$. Then $\mathfrak{J}_{M} / n \subseteq \mathfrak{J}_{M} / m$ (see Lemma 5.2). Moreover, $\left(\mathfrak{J}_{M} / n\right)_{M_{0}, \alpha}^{+} \subseteq\left(\mathfrak{J}_{M} / m\right)_{M_{0}, \alpha}^{+}$thanks to Lemma 6.6. Therefore if $\psi$ is positive with respect to the cone $\left(\mathfrak{J}_{M} / m\right)_{M_{0}, \alpha}^{+}$, then it is positive with respect to the cone $\left(\mathfrak{J}_{M} / n\right)_{M_{0}, \alpha}^{+}$too. Therefore $\psi(T / n)(x, y)=\left\langle\Psi_{m, n}(T) x, y\right\rangle_{n}, x, y \in \Delta$, for the uniquely determined $\alpha$-positive linear mapping $\Psi_{m, n}: \mathfrak{J}_{M} \rightarrow \mathcal{B}\left(\Delta_{n}\right)$. It follows that

$$
\left\langle\Psi_{m, n}(T) x, y\right\rangle_{n}=\psi\left(T n^{-1} m / m\right)(x, y)=\left\langle\Psi_{m}\left(T n^{-1} m\right) x, y\right\rangle_{m}
$$


for all $x, y \in \Delta$. In particular,

$$
\begin{aligned}
\left\langle\Psi_{m, n}^{(k)}(T) x, y\right\rangle_{n}^{(k)} & =\psi^{(k)}\left(T /\left(n \cdot 1_{H^{k}}\right)\right)(x, y) \\
& =\psi^{(k)}\left(T\left(n^{-1} m \cdot 1_{H^{k}}\right) /\left(m \cdot 1_{H^{k}}\right)\right)(x, y) \\
& =\left\langle\Psi_{m}^{(k)}\left(T\left(n^{-1} m \cdot 1_{H^{k}}\right)\right) x, y\right\rangle_{m}^{(k)}
\end{aligned}
$$

for all $T \in \mathbb{M}_{k}\left(\mathfrak{J}_{M}\right), x, y \in \Delta^{k}, k \in \mathbb{N}$. Thus $\psi_{n}$ is matrix $\alpha$-contractive iff $\Psi_{m, n}$ is as well. By analogy, $\Psi_{m, n}$ is $n$-fractionally matrix $\alpha$-positive iff $\psi_{n}$ is matrix $\alpha$-positive.

Remark 7.1. Let $\psi: \mathfrak{J}_{M} / M \rightarrow S F(\Delta)$ be an inner-product mapping which is $M_{0^{-}}$ fractionally $\alpha$-positive. Put $\psi_{m, x}: \mathfrak{J}_{M} / m \rightarrow \mathbb{C}, \psi_{m, x}(T / m)=\psi_{m}(T / m)(x, x)$, which is a linear functional. Using Corollary 7.1 we infer that

$$
\begin{aligned}
\psi_{m, x}\left(1_{H} / m\right) & =\psi\left(1_{H} / m\right)(x, x)=\sup \left\{|\psi(T / m)(x, x)|: T \in \mathfrak{J}_{M},\|T\|_{\alpha} \leq 1\right\} \\
& =\sup \left\{\left|\psi_{m, x}(b)\right|: b \in \mathfrak{J}_{M} / m,\|b\|_{m, \alpha} \leq 1\right\} \\
& =\left\|\psi_{m, x}\right\|_{m, \alpha}
\end{aligned}
$$

for all $m \in M_{0}, x \in \Delta$. If $\mathcal{F}_{M}=\sum_{m \in M} \mathcal{F}_{m}$ is a fractional space in $\mathfrak{J}_{M} / M$ and $\phi: \mathcal{F}_{M} \rightarrow S F(\Delta)$ is a unital linear mapping, then we shall use the notation $\phi_{m}=\phi \mid \mathcal{F}_{m}$ and $\phi_{m, x}: \mathcal{F}_{m} \rightarrow \mathbb{C}, \phi_{m, x}(b)=\phi_{m}(b)(x, x), x \in \Delta, m \in M_{0}$.

Let $\psi: \mathfrak{J}_{M} / M \rightarrow S F(\Delta)$ be an inner-product mapping which is matrix $\alpha$ positive. By its very definition, all restrictions $\psi_{m}, m \in M_{0}$, are matrix $\alpha$-positive for a certain cofinal subset $M_{0} \subseteq M$. By Lemma 7.5,

$$
\psi(T / m)(x, y)=\left\langle\Psi_{m}(T) x, y\right\rangle_{m}, \quad x, y \in \Delta,
$$

for the uniquely determined unital $m$-fractionally matrix $\alpha$-positive mappings $\Psi_{m}$ : $\mathfrak{J}_{M} \rightarrow \mathcal{B}\left(\Delta_{m}\right), m \in M_{0}$. If $K=\Delta_{1_{H}}\left(m=1_{H} \in M_{0}\right)$ and $\Psi=\Psi_{1_{H}}$, then $\Psi: \mathfrak{J}_{M} \rightarrow \mathcal{B}(K)$ is a unital matrix $\alpha$-positive mapping (see Subsection 7.2).

Proposition 7.2. If $\psi: \mathfrak{J}_{M} / M \rightarrow S F(\Delta)$ is a quantum $\Delta$-measure on the $C^{*}$ algebra $\mathfrak{J}_{M}$ with support in $H_{\alpha}$, then the mapping $\Psi=\Psi_{1_{H}}: \mathfrak{J}_{M} \rightarrow \mathcal{B}(K)$ determines a quantum $\mathcal{B}(K)$-valued measure on $\mathfrak{J}_{M}$ with support in $H_{\alpha}$ such that $\psi$ is a sesquilinear extension of $\Psi$.

Proof. By Theorem 7.1, $\psi: \mathfrak{J}_{M} / M \rightarrow S F(\Delta)$ is a matrix $\alpha$-positive inner-product mapping. We have to prove that $\psi$ is a sesquilinear extension of $\Psi$ (see Definition 7.3). We set $\mu_{x, y}(T)=\langle\Psi(T) x, y\rangle$ for $T \in \mathfrak{J}_{M}$ and $x, y \in \Delta$. One may extend $\mu_{x, y}$ to the rational functions from $\mathfrak{J}_{M} / M$ in the following way. Take $T / m \in \mathfrak{J}_{M} / m$. Put

$$
\widetilde{\mu}_{x, y}(T / m)=\left\langle\Psi_{m}(T) x, y\right\rangle_{m}=\psi(T / m)(x, y) .
$$

If $m=1_{H}$, then $\widetilde{\mu}_{x, y}\left(T / 1_{H}\right)=\left\langle\Psi_{1_{H}}(T) x, y\right\rangle=\langle\Psi(T) x, y\rangle=\mu_{x, y}(T)$; that is, $\widetilde{\mu}_{x, y}$ extends $\mu_{x, y}$. If $n \preceq m, n, m \in M_{0}$, then $\widetilde{\mu}_{x, y}(T / n)=\psi(T / n)(x, y)=$ $\psi\left(T n^{-1} m / m\right)(x, y)=\widetilde{\mu}_{x, y}\left(T\left(n^{-1} m\right) / m\right)$. Moreover,

$$
\widetilde{\mu}_{x, y}(T m / m)=\psi(T m / m)(x, y)=\psi(T n / n)(x, y)=\widetilde{\mu}_{x, y}(T n / n),
$$

whence $\widetilde{\mu}_{x, y}(T m / m)=\widetilde{\mu}_{x, y}\left(T / 1_{H}\right)=\mu_{x, y}(T)$. Thus all $\widetilde{\mu}_{x, y}$ are well defined extensions of $\mu_{x, y}$, respectively. Obviously, the sesquilinear form $\Delta \times \Delta \rightarrow \mathbb{C}$, 
$(x, y) \mapsto \widetilde{\mu}_{x, y}(b)$ is just $\psi(b)$ for each $b \in \mathfrak{J}_{M} / M$. Consequently, $\psi: \mathfrak{J}_{M} / M \rightarrow$ $S F(\Delta)$ is a sesquilinear extension of $\Psi$.

7.5. The admissible inner product mapping. Let $\mathcal{F}_{M}=\sum_{m \in M} \mathcal{F}_{m}$ be a fractional space in $\mathfrak{J}_{M} / M$ and let $\phi: \mathcal{F}_{M} \rightarrow S F(\Delta)$ be an inner-product mapping. So, we have a family $\Delta_{m}, m \in M_{0}$, of Hilbert space completions of $\Delta$ (see Definition 7.1). The following definition plays a crucial role.

Definition 7.4. We say that $\phi: \mathcal{F}_{M} \rightarrow S F(\Delta)$ is an $\alpha$-admissible inner product mapping if $\phi_{m}(T / m)(x, y)=\left\langle\Psi_{m}(T) x, y\right\rangle_{m}$ for some $m$-fractionally matrix $\alpha$ positive mapping $\Psi_{m}: \mathfrak{J}_{M} \rightarrow \mathcal{B}\left(\Delta_{m}\right)$ for each $m \in M_{0}$.

If $\mu: \mathfrak{J}_{M} / M \rightarrow S F(\Delta)$ is a quantum $\Delta$-measure on $\mathfrak{J}_{M}$ with support in $H_{\alpha}$, then $\mu$ is a matrix $\alpha$-positive inner product mapping by Theorem 7.1. In particular, each $\mu_{m}$ is matrix $\alpha$-positive. Using Lemma 7.5, we derive that $\mu_{m}(T / m)(x, y)=$ $\left\langle\Psi_{m}(T) x, y\right\rangle_{m}$ for some $m$-fractionally matrix $\alpha$-positive mapping $\Psi_{m}: \mathfrak{J}_{M} \rightarrow$ $\mathcal{B}\left(\Delta_{m}\right)$, thereby $\mu$ is $\alpha$-admissible.

Lemma 7.6. Let $\mathcal{F}_{M}=\sum_{m \in M} \mathcal{F}_{m}$ be a fractional space in $\mathfrak{J}_{M} / M$ and let $\phi$ : $\mathcal{F}_{M} \rightarrow S F(\Delta)$ be a matrix $\alpha$-contractive inner-product mapping. There are matrix $\alpha$-positive and matrix $\alpha$-contractive mappings $\Psi_{m}: \mathfrak{J}_{M} \rightarrow \mathcal{B}\left(\Delta_{m}\right)$ such that

$$
\phi_{m}(T / m)(x, y)=\left\langle\Psi_{m}(T) x, y\right\rangle_{m}
$$

for all $T / m \in \mathcal{F}_{m}, m \in M_{0}$, where $M_{0}$ is a unital cofinal subset in $M$.

Proof. Since $\phi$ is an inner-product mapping, we have a unital cofinal subset $M_{0} \subseteq$ $M$. Fix $m \in M_{0}$. If $T / m \in \mathcal{F}_{m}$, then $\left|\phi_{m}(T / m)(x, y)\right| \leq\left\|\phi_{m}(T / m)\right\|_{m}^{(1)}\|x\|_{m}\|y\|_{m}$ $\leq\left\|\phi_{m}\right\|_{m, \alpha}^{(1)}\|T\|_{\alpha}\|x\|_{m}\|y\|_{m} \leq\left\|\phi_{m}\right\|_{m, \alpha}^{c b}\|T\|_{\alpha}\|x\|_{m}\|y\|_{m} \leq\|T\|_{\alpha}\|x\|_{m}\|y\|_{m}$. Using the density of $\Delta$ in $\Delta_{m}$, infer that $\phi_{m}(T / m)$ is a bounded form on $\Delta_{m}$. There exists a unique operator $\Phi_{m}^{\prime}\left(T \mid H_{\alpha}\right) \in \mathcal{B}\left(\Delta_{m}\right)$ such that

$$
\phi_{m}(T / m)(x, y)=\left\langle\Phi_{m}^{\prime}\left(T \mid H_{\alpha}\right) x, y\right\rangle_{m}=\phi\left(1_{H} / m\right)\left(\Phi_{m}^{\prime}\left(T \mid H_{\alpha}\right) x, y\right)
$$

for all $x, y \in \Delta$. Thus we have a unital linear mapping $\Phi_{m}: \mathcal{F}^{(m)} \rightarrow \mathcal{B}\left(\Delta_{m}\right)$, $\Phi_{m}(T)=\Phi_{m}^{\prime}\left(T \mid H_{\alpha}\right)$, where $\mathcal{F}^{(m)}=\left\{T \in \mathfrak{J}_{M}: T / m \in \mathcal{F}_{m}\right\}$ is a subspace in $\mathfrak{J}_{M}$. With $\left\|\phi_{m}\right\|_{m, \alpha}^{c b} \leq 1$ in mind, we infer that $\Phi_{m}$ is a matrix $\alpha$-contraction. Indeed, $\phi_{m}^{(k)}\left(T /\left(m \cdot 1_{H^{k}}\right)\right)(x, y)=\left\langle\Phi_{m}^{(k)}(T) x, y\right\rangle_{m}^{(k)}$ for all $T=\left[T_{i j}\right] \in C_{\mathcal{E}^{k}}^{*}\left(H^{k}\right)$, $T_{i j} / m \in \mathcal{F}_{m}, x=\left[x_{i}\right], y=\left[y_{i}\right] \in \Delta^{k}$. In particular, $\left\|\phi_{m}^{(k)}\left(T /\left(m \cdot 1_{H^{k}}\right)\right)\right\|_{m}^{(k)}=$ $\left\|\Phi_{m}^{(k)}(T)\right\|_{\mathcal{B}\left(\Delta_{m}^{k}\right)}$. It follows that

$$
\begin{aligned}
\left\|\Phi_{m}^{(k)}\right\|_{\alpha}^{(k)} & =\sup \left\{\left\|\Phi_{m}^{(k)}(T)\right\|_{\mathcal{B}\left(\Delta_{m}^{k}\right)}: T \in \mathbb{M}_{k}\left(\mathcal{F}^{(m)}\right),\left\|T \mid H_{\alpha}^{k}\right\| \leq 1\right\} \\
& =\sup \left\{\left\|\phi^{(k)}\left(T /\left(m \cdot 1_{H^{k}}\right)\right)\right\|_{m}^{(k)}: T /\left(m \cdot 1_{H^{k}}\right) \in \mathbb{M}_{k}\left(\mathcal{F}_{m}\right),\|T\|_{\alpha}^{(k)} \leq 1\right\} \\
& =\left\|\phi_{m}^{(k)}\right\|_{m, \alpha}^{(k)} .
\end{aligned}
$$

In particular, $\left\|\Phi_{m}\right\|_{\alpha}^{c b}=\sup \left\{\left\|\Phi_{m}^{(k)}\right\|_{\alpha}^{(k)}: k \in \mathbb{N}\right\}=\left\|\phi_{m}\right\|_{m, \alpha}^{c b} \leq 1$. Therefore, $\Phi_{m}$ is a matrix $\alpha$-contraction. It follows that it can be extended up to a matrix $\alpha$ contractive mapping $\Psi_{m}: \mathfrak{J}_{M} \rightarrow \mathcal{B}\left(\Delta_{m}\right)$ thanks to the Hahn-Banach theorem [26, 
Theorem 2.3.1] (see also [9]). Since $\mathfrak{J}_{M}$ is a normed quantum system (or operator system) on $H$ and $\Psi_{m}$ is unital, it follows that $\Psi_{m}$ is matrix $\alpha$-positive too [12, $5.1 .2], 9$.

Lemma 7.7. If $\phi: \mathcal{F}_{M} \rightarrow S F(\Delta)$ is an $\alpha$-admissible inner product mapping, then $\phi$ is matrix $\alpha$-contractive.

Proof. By Definition 7.4, there is an $m$-fractionally matrix $\alpha$-positive mapping $\Psi_{m}$ : $\mathfrak{J}_{M} \rightarrow \mathcal{B}\left(\Delta_{m}\right)$ such that $\phi_{m}(T / m)(x, y)=\left\langle\Psi_{m}(T) x, y\right\rangle_{m}$. Evidently, $m$-fractionally matrix $\alpha$-positivity involves matrix $\alpha$-positivity. Being a unital mapping, each $\Psi_{m}$ is matrix $\alpha$-contractive [9], [12, 5.1.2]. As in the proof of Lemma 7.6, we have $\left\|\phi_{m}^{(k)}\left(T /\left(m \cdot 1_{H^{k}}\right)\right)\right\|_{m}^{(k)}=\left\|\Psi_{m}^{(k)}(T)\right\|_{\mathcal{B}\left(\Delta_{m}^{k}\right)} ;$ that is,

$$
\begin{aligned}
\left\|\phi_{m}^{(k)}\right\|_{m, \alpha}^{(k)} & =\sup \left\{\left\|\phi^{(k)}\left(T /\left(m \cdot 1_{H^{k}}\right)\right)\right\|_{m}^{(k)}: T /\left(m \cdot 1_{H^{k}}\right) \in \mathbb{M}_{k}\left(\mathcal{F}_{m}\right),\|T\|_{\alpha}^{(k)} \leq 1\right\} \\
& \leq\left\|\Psi_{m}^{(k)}\right\|_{\alpha}^{(k)} .
\end{aligned}
$$

The assumption $T /\left(m \cdot 1_{H^{k}}\right) \in \mathbb{M}_{k}\left(\mathcal{F}_{m}\right)$ just restricts the set of those $T$ in the latter inequality. In particular,

$$
\left\|\phi_{m}\right\|_{m, \alpha}^{c b}=\sup \left\{\left\|\phi_{m}^{(k)}\right\|_{m, \alpha}^{(k)}: k \in \mathbb{N}\right\} \leq \sup \left\{\left\|\Psi_{m}^{(k)}\right\|_{\alpha}^{(k)}: k \in \mathbb{N}\right\}=\left\|\Psi_{m}\right\|_{\alpha}^{c b} \leq 1 .
$$

Therefore, $\phi_{m}$ is a matrix $\alpha$-contraction for each $m \in M_{0}$.

Note that we cannot assert that a matrix $\alpha$-contractive inner-product mapping is automatically $\alpha$-admissible. By Lemma 7.6 , each mapping $\Psi_{m}: \mathfrak{J}_{M} \rightarrow \mathcal{B}\left(\Delta_{m}\right)$ is matrix $\alpha$-positive and matrix $\alpha$-contractive. But we do not know whether $\Psi_{m}$ is $m$-fractionally matrix $\alpha$-positive. That depends on the particular choice of the mapping $\phi$ or the $C^{*}$-algebra $\mathfrak{J}_{M}$ related to the denominator set $M$. In the commutative case each matrix $\alpha$-contractive mapping $\phi: \mathcal{F}_{M} \rightarrow S F(\Delta)$ is admissible. All details will be presented in the next section. The following noncommutative case presents an interest to be considered too.

Theorem 7.2. Let $\mathcal{F}_{M}=\sum_{m \in M} \mathcal{F}_{m}$ be a fractional space in $\mathfrak{J}_{M} / M$ and let $\phi$ : $\mathcal{F}_{M} \rightarrow S F(\Delta)$ be a matrix $\alpha$-contractive inner-product mapping. Assume that $m / n^{2} \in \mathcal{F}_{M}$ and that there is a positive number $\lambda_{n, m}$ with

$$
\lambda_{n, m} \phi(1 / m)=\phi(1 / n)=\frac{1}{\lambda_{n, m}} \phi\left(m / n^{2}\right)
$$

whenever $n \preceq m, n, m \in M_{0}$. Then $\phi$ is $\alpha$-admissible.

Proof. By Lemma 7.6, $\phi_{m}(T / m)(x, y)=\left\langle\Psi_{m}(T) x, y\right\rangle_{m}$ for a matrix $\alpha$-positive and matrix $\alpha$-contractive mapping $\Psi_{m}: \mathfrak{J}_{M} \rightarrow \mathcal{B}\left(\Delta_{m}\right), m \in M_{0}$. Then

$$
\begin{aligned}
\left\langle\Psi_{m}\left(n^{-1} m\right) x, y\right\rangle_{m} & =\phi\left(\left(n^{-1} m\right) / m\right)(x, y)=\phi(1 / n)(x, y) \\
& =\langle x, y\rangle_{n}=\lambda_{n, m} \phi(1 / m)(x, y) \\
& =\lambda_{n, m}\langle x, y\rangle_{m}=\left\langle\lambda_{n, m} x, y\right\rangle_{m}
\end{aligned}
$$


for all $x, y \in \Delta$. Using the density of $\Delta$ in $\Delta_{m}$, we infer that $\Psi_{m}\left(n^{-1} m\right)=$ $\lambda_{n, m} 1_{\Delta_{m}}$. Further, note that

$$
\begin{aligned}
\left(m n^{-1}\right) \mid H_{\beta} & =\left(m \mid H_{\beta}\right)\left(n \mid H_{\beta}\right)^{-1}=\left(\left(n \mid H_{\beta}\right)^{-1}\left(m \mid H_{\beta}\right)\right)^{*} \\
& =\left(\left(n^{-1} m\right) \mid H_{\beta}\right)^{*}=\left(n^{-1} m\right)^{*} \mid H_{\beta}
\end{aligned}
$$

for all $\beta \in \Lambda$. Whence $\left(n^{-1} m\right)^{*}=m n^{-1}$. With $\left(n^{-1} m\right)^{*} \in \mathfrak{J}_{M}$ in mind, we infer that

$$
\begin{aligned}
\left\langle\Psi_{m}\left(\left(n^{-1} m\right)^{*}\left(n^{-1} m\right)\right) x, y\right\rangle_{m} & =\phi\left(m n^{-2} m / m\right)(x, y)=\phi\left(m / n^{2}\right)(x, y) \\
& =\lambda_{n, m} \phi(1 / n)(x, y)=\lambda_{n, m}^{2} \phi(1 / m)(x, y) \\
& =\left\langle\lambda_{n, m}^{2} x, y\right\rangle_{m}
\end{aligned}
$$

for all $x, y \in \Delta$. Whence $\Psi_{m}\left(\left(n^{-1} m\right)^{*}\left(n^{-1} m\right)\right)=\lambda_{n, m}^{2} 1_{\Delta_{m}}=\Psi_{m}\left(n^{-1} m\right)^{2}$. Using [12, Corollary 5.2.2] (see also [9, Corollary 5.5]), we conclude that

$$
\Psi_{m}\left(T\left(n^{-1} m\right)\right)=\Psi_{m}(T) \Psi_{m}\left(n^{-1} m\right)
$$

whenever $T \in \mathfrak{J}_{M}$ and $n \preceq m, n, m \in M_{0}$.

Now consider the matrices over $\mathfrak{J}_{M}$. Take $T=\left[T_{i j}\right] \in \mathbb{M}_{k}\left(\mathfrak{J}_{M}\right)$. Then

$$
\begin{aligned}
\Psi_{m}^{(k)}\left(T\left(n^{-1} m \cdot 1_{H^{k}}\right)\right) & =\Psi_{m}^{(k)}\left[T_{i j}\left(n^{-1} m\right)\right]=\left[\Psi_{m}\left(T_{i j}\left(n^{-1} m\right)\right)\right] \\
& =\left[\Psi_{m}\left(T_{i j}\right) \Psi_{m}\left(n^{-1} m\right)\right] \\
& =\lambda_{n, m} \Psi_{m}^{(k)}(T) .
\end{aligned}
$$

If $T \geq_{\alpha} 0$ in $\mathbb{M}_{k}\left(\mathfrak{J}_{M}\right)$, then $\Psi_{m}^{(k)}(T) \geq 0$, for $\Psi_{m}$ is matrix $\alpha$-positive. Hence $\Psi_{m}^{(k)}\left(T\left(n^{-1} m \cdot 1_{H^{k}}\right)\right)=\lambda_{n, m} \Psi_{m}^{(k)}(T) \geq 0$; that is, $\Psi_{m}$ is $m$-fractionally matrix $\alpha$-positive. By Definition 7.4, we conclude that $\phi$ is $\alpha$-admissible.

The following corollary of the just-proved assertion will be used in Section 9 ,

Corollary 7.2. Let $\mathcal{F}_{M}=\sum_{m \in M} \mathcal{F}_{m}$ be a fractional space in $\mathfrak{J}_{M} / M$ and let $\phi: \mathcal{F}_{M} \rightarrow \mathbb{C}$ be a unital $\alpha$-contractive functional. Assume that $m / n^{2} \in \mathcal{F}_{M}$ and

$$
\phi(1 / n)^{2}=\phi(1 / m) \phi\left(m / n^{2}\right) \quad \text { and } \quad \phi(1 / m)>0
$$

whenever $n \preceq m, n, m \in M_{0}$. Then $\phi$ is $\alpha$-admissible.

Proof. Note that $\phi: \mathcal{F}_{M} \rightarrow \mathbb{C}$ is automatically matrix $\alpha$-contractive. It remains to use Theorem 7.2 ,

7.6. Noncommutative Albrecht-Vasilescu theorem. In this subsection we propose a noncommutative version of the extension result proved in 2, Theorem 2.5] by E. Albrecht and F.-H. Vasilescu.

Theorem 7.3. Let $M$ be a unital upward filtered set of denominators, $\mathcal{F}_{M}=$ $\sum_{m \in M} \mathcal{F}_{m}$ a fractional space in $\mathfrak{J}_{M} / M$ and let $\phi: \mathcal{F}_{M} \rightarrow S F(\Delta)$ be an inner product mapping. The mapping $\phi$ extends up to a quantum $\Delta$-measure $\psi: \mathfrak{J}_{M} / M \rightarrow$ $S F(\Delta)$ with support in $H_{\alpha}$ such that $\left\|\psi_{m, x}\right\|_{m, \alpha}=\left\|\phi_{m, x}\right\|_{m, \alpha}, m \in M_{0}, x \in \Delta$, if and only if $\phi$ is $\alpha$-admissible, where $M_{0} \subseteq M$ is a unital cofinal subset. In particular, $\phi$ is a matrix $\alpha$-contraction. 
Proof. First, assume that $\phi$ has an extension up to a quantum $\Delta$-measure $\psi$ : $\mathfrak{J}_{M} / M \rightarrow S F(\Delta)$ with support in $H_{\alpha}$ such that $\left\|\psi_{m, x}\right\|_{m, \alpha}=\left\|\phi_{m, x}\right\|_{m, \alpha}$ for all $m \in M_{0}, x \in \Delta$. By Theorem 7.1, $\psi: \mathfrak{J}_{M} / M \rightarrow S F(\Delta)$ is a matrix $\alpha$-positive mapping such that $\psi\left(\mathfrak{J}_{M} / m\right) \subseteq S F_{m}(\Delta), m \in M_{0}$. In particular, $\psi$ is an $M_{0^{-}}$ fractionally $\alpha$-positive mapping. As we have confirmed in Remark 7.1,

$$
\left\|\psi_{m, x}\right\|_{m, \alpha}=\psi_{m, x}\left(1_{H} / m\right)=\psi\left(1_{H} / m\right)(x, x)=\phi\left(1_{H} / m\right)(x, x)
$$

(see also Definition 5.2). Using Lemma 7.5, we infer that

$$
\psi(T / m)(x, y)=\left\langle\Psi_{m}(T) x, y\right\rangle_{m}, \quad x, y \in \Delta,
$$

for the uniquely determined unital $m$-fractionally matrix $\alpha$-positive mapping $\Psi_{m}$ : $\mathfrak{J}_{M} \rightarrow \mathcal{B}\left(\Delta_{m}\right)$. It follows that $\phi$ is $\alpha$-admissible (see Definition 7.4). In particular, $\phi$ is matrix $\alpha$-contractive thanks to Lemma 7.7

Now assume that $\phi: \mathcal{F}_{M} \rightarrow S F(\Delta)$ is an $\alpha$-admissible inner product mapping. So, there is an $m$-fractionally matrix $\alpha$-positive mapping $\Psi_{m}: \mathfrak{J}_{M} \rightarrow \mathcal{B}\left(\Delta_{m}\right)$ such that $\phi_{m}(T / m)(x, y)=\left\langle\Psi_{m}(T) x, y\right\rangle_{m}, T / m \in \mathcal{F}_{m}, x, y \in \Delta$, for each $m \in M_{0}$. In particular, for the fixed $m \in M_{0}$ we have a matrix $\alpha$-positive mapping

$$
\gamma_{m}: \mathfrak{J}_{M} / m \rightarrow S F_{M_{0}}(\Delta), \quad \gamma_{m}(T / m)(x, y)=\left\langle\Psi_{m}(T) x, y\right\rangle_{m}
$$

(see Lemma [7.5). Take $n \in M_{0}$ with $n \preceq m$. Then $1_{H} / n \in \mathcal{F}_{n} \subseteq \mathcal{F}_{m}$ (see Definition [5.2) and $1_{H} / n \in\left(\mathfrak{J}_{M} / n\right)_{M_{0}, \alpha}^{+} \subseteq\left(\mathfrak{J}_{M} / m\right)_{M_{0}, \alpha}^{+}$thanks to Lemma 6.6. Since $\phi_{n}=\phi_{m} \mid \mathcal{F}_{n}$, it follows that

$$
\begin{aligned}
\gamma_{m}\left(1_{H} / n\right)(x, x) & =\gamma_{m}\left(n^{-1} m / m\right)(x, x)=\left\langle\Psi_{m}\left(n^{-1} m\right) x, x\right\rangle_{m} \\
& =\phi_{m}\left(n^{-1} m / m\right)(x, x) \\
& =\phi_{n}\left(1_{H} / n\right)(x, x)>0
\end{aligned}
$$

for all $x \in \Delta \backslash\{0\}$. Using the same argument as in proof of Lemma 7.5, we derive that there is a unital $n$-fractionally matrix $\alpha$-positive mapping $\Psi_{m, n}: \mathfrak{J}_{M} \rightarrow \mathcal{B}\left(\Delta_{n}\right)$ such that

$$
\left\langle\Psi_{m, n}(T) x, y\right\rangle_{n}=\gamma_{m}(T / n)(x, y)=\left\langle\Psi_{m}\left(T n^{-1} m\right) x, y\right\rangle_{m}
$$

for all $T \in \mathfrak{J}_{M}, x, y \in \Delta$. In particular,

$$
\gamma_{m, n}: \mathfrak{J}_{M} / n \rightarrow S F(\Delta), \quad \gamma_{m, n}(T / n)(x, y)=\left\langle\Psi_{m, n}(T) x, y\right\rangle_{n}
$$

is a unital matrix $\alpha$-positive mapping such that

$$
\gamma_{m, n}=\gamma_{m} \mid \mathfrak{J}_{M} / n
$$

and

$$
\begin{aligned}
\gamma_{m, n}(T / n)(x, y) & =\left\langle\Psi_{m}\left(T n^{-1} m\right) x, y\right\rangle_{m}=\phi_{m}\left(T n^{-1} m / m\right)(x, y) \\
& =\phi_{n}(T / n)(x, y)
\end{aligned}
$$

whenever $T / n \in \mathcal{F}_{n}, x, y \in \Delta$; that is,

$$
\gamma_{m, n} \mid \mathcal{F}_{n}=\phi_{n} .
$$

Now we use the trick proposed in [2, Theorem 2.5] (see also [19]). Fix $m \in M_{0}$ and consider the set $K_{m}$ of all complex-valued functions $a: \mathfrak{J}_{M} / m \times \Delta \times \Delta \rightarrow \mathbb{C}$ such that

$$
|a(T / m, x, y)| \leq\|T\|_{\alpha}\|x\|_{m}\|y\|_{m}
$$


for all $(T / m, x, y) \in \mathfrak{J}_{M} / m \times \Delta \times \Delta$. Evidently, the set $K_{m}$ is a compact space with respect to the pointwise convergence topology. Therefore $K=\prod_{m \in M_{0}} K_{m}$ is a compact topological space. Consider its subspace $\Omega_{m} \subseteq K$ comprising those $a=\left(a_{n}\right)_{n \in M_{0}} \in K$ such that for each $n \in M_{0}, n \preceq m$, the mapping $a_{n}$ : $\mathfrak{J}_{M} / m \rightarrow S F(\Delta), a_{n}(T / m)(x, y)=a_{n}(T / m, x, y)$, is matrix $\alpha$-positive with $a_{n}=a_{m} \mid\left(\mathfrak{J}_{M} / n \times \Delta \times \Delta\right)$ and $a_{n} \mid \mathcal{F}_{n}=\phi_{n}$. Then $\Omega_{m}$ is closed, and therefore it is a compact space. Moreover, as we have just proved above, $\omega=\left(\omega_{n}\right)_{n \in M_{0}} \in \Omega_{m}$, where $\omega_{m}=\gamma_{m}, \omega_{n}=\gamma_{m, n}$ if $n \preceq m$, and $\omega_{n}=0$ otherwise (see (7.3) and (7.4)). Further, if $m \preceq s$ for some $m, s \in M_{0}$, then $\Omega_{s} \subseteq \Omega_{m}$. Indeed, if $a \in \Omega_{s}$ and $n \preceq m$, then $n \preceq s$. Therefore

$$
\begin{aligned}
a_{m} \mid\left(\mathfrak{J}_{M} / n \times \Delta \times \Delta\right) & =\left(a_{s} \mid\left(\mathfrak{J}_{M} / m \times \Delta \times \Delta\right)\right) \mid\left(\mathfrak{J}_{M} / n \times \Delta \times \Delta\right) \\
& =a_{s} \mid\left(\mathfrak{J}_{M} / n \times \Delta \times \Delta\right)=a_{n}
\end{aligned}
$$

and $a_{n} \mid \mathcal{F}_{n}=\phi_{n}$. Whence $a \in \Omega_{m}$.

Taking into account the fact that $M_{0}$ is a cofinal subset of an upward filtered set, we deduce that $\left\{\Omega_{m}: m \in M_{0}\right\}$ is a filter base in the compact space $K$. Therefore the set $\bigcap_{m \in M_{0}} \Omega_{m}$ being the set of all limit points of the filter associated with the indicated filter base is nonempty [3, 1.9.1]. Take $b=\left(b_{n}\right)_{n \in M_{0}} \in \bigcap_{m \in M_{0}} \Omega_{m}$. Then $b_{m} \mid\left(\mathfrak{J}_{M} / n \times \Delta \times \Delta\right)=b_{n}$ and $b_{n} \mid \mathcal{F}_{n}=\phi_{n}$ whenever $n \preceq m, n, m \in M_{0}$. We put

$$
\psi(u)(x, y)=b_{m}(u, x, y)
$$

if $u \in \mathfrak{J}_{M} / m, x, y \in \Delta$. If $u \in \mathfrak{J}_{M} / n \cap \mathfrak{J}_{M} / s$ for some $n, s \in M_{0}$, then $\mathfrak{J}_{M} / n+$ $\mathfrak{J}_{M} / s \subseteq \mathfrak{J}_{M} / m$ for a certain $m \in M_{0}$, for $M$ is upward filtered and $M_{0}$ is its cofinal subset. With $b \in \Omega_{m}$ in mind, we infer that

$$
b_{n}(u, x, y)=b_{m}(u, x, y)=b_{s}(u, x, y)
$$

for all $x, y \in \Delta$, and the mapping $(x, y) \rightarrow b_{m}(u, x, y)$ is a sesquilinear form. Thus $\psi: \mathfrak{J}_{M} / M \rightarrow S F(\Delta)$ is a well defined matrix $\alpha$-positive mapping extending $\phi$. Using Theorem 7.1 and Lemma 7.7. we derive that $\psi\left(\mathfrak{J}_{M} / m\right) \subseteq S F_{m}(\Delta), m \in M_{0}$, and $\psi$ is a matrix $\alpha$-contraction. Hence

$$
\begin{aligned}
\left\|\phi_{m, x}\right\|_{m, \alpha} & \leq\left\|\psi_{m, x}\right\|_{m, \alpha} \leq\left\|\psi\left(1_{H} / m\right)\right\|_{m}\|x\|_{m}^{2} \leq\|x\|_{m}^{2} \\
& =\phi\left(1_{H} / m\right)(x, x)=\phi_{m, x}\left(1_{H} / m\right) \\
& \leq\left\|\phi_{m, x}\right\|_{m, \alpha} ;
\end{aligned}
$$

that is, $\left\|\phi_{m, x}\right\|_{m, \alpha}=\left\|\psi_{m, x}\right\|_{m, \alpha}$ for all $m \in M_{0}, x \in \Delta$.

Remark 7.2. As we have mentioned in the introduction, the functional scheme of the fractional space technique developed in [2], 24] and 25] can be considered as a particular case of the proposed above quantum construction. The space $H_{\alpha}$ represents the square integrable functions with support in a compact set $K_{\alpha}$. Keeping the support of a quantum $\Delta$-measure $\psi: \mathfrak{J}_{M} / M \rightarrow S F(\Delta)$ in $H_{\alpha}$, just proving Theorem 7.3 effectively generalizes the relevant functional extension result proved in [2, Theorem 2.5] (see Corollary 8.1] below). 


\section{The Mutually COMmuting Denominators}

In this section we investigate the fractional spaces associated with a set of mutually commuting positive denominators, and using the noncommutative AlbrechtVasilescu theorem we solve the quantum moment problem for this case.

8.1. The $C^{*}$-algebra $M_{\mathcal{E}}^{\prime}$ related to $M$. Let $M \subseteq \mathfrak{M}_{\mathcal{E}}$ be a subset. We say that $M$ is a commutative set of denominators in $\mathfrak{M}_{\mathcal{E}}$ if $M$ consists of mutually commuting positive operators from $\mathfrak{M}_{\mathcal{E}}$ with $1_{H} \in M$. We set $M_{\mathcal{E}}^{\prime}$ to denote the commutant of the set $M$ in the $C^{*}$-algebra $C_{\mathcal{E}}^{*}(H)$; that is, $M_{\mathcal{E}}^{\prime}=M^{\prime} \cap C_{\mathcal{E}}^{*}(H)$, where $M^{\prime}$ is the commutant of $M$ in $\mathcal{B}(H)$. Since all operators from $M$ are normal, it follows that (see for instance [20, 12.12.16]) $M_{\mathcal{E}}^{\prime}$ is a unital $C^{*}$-algebra in $C_{\mathcal{E}}^{*}(H)$. Taking into account that $M$ is itself commutative, we conclude that $M \subseteq M_{\mathcal{E}}^{\prime}$. For each $k \in \mathbb{N}$ the set $M^{k}=\left\{m \cdot 1_{H^{k}}: m \in M\right\}$ is a commutative set of denominators in $C_{\mathcal{E}^{k}}^{*}\left(H^{k}\right)$. Its commutant in $C_{\mathcal{E}^{k}}^{*}\left(H^{k}\right)$ is denoted by $M_{\mathcal{E}^{k}}^{\prime}$. If $T=\left[T_{i j}\right] \in M_{\mathcal{E}^{k}}^{\prime}$, then $\left[T_{i j} m\right]=T\left(m \cdot 1_{H^{k}}\right)=\left(m \cdot 1_{H^{k}}\right) T=\left[m T_{i j}\right]$ for all $m \in M$. Hence

$$
M_{\mathcal{E}^{k}}^{\prime}=\mathbb{M}_{k}\left(M_{\mathcal{E}}^{\prime}\right)
$$

for all $k$.

Lemma 8.1. Let $M$ be a commutative set of denominators in $\mathfrak{M}_{\mathcal{E}}$. If $n \preceq m$ for some $n, m \in M$, then $n^{-1} m \geq 0$ in the $C^{*}$-algebra $C_{\mathcal{E}}^{*}(H)$. Moreover, $n^{-1} m \in M_{\mathcal{E}}^{\prime}$. Thus $M_{\mathcal{E}}^{\prime}$ is a $C^{*}$-algebra in $C_{\mathcal{E}}^{*}(H)$ related to the set $M$.

Proof. Take $\alpha \in \Lambda$. Since $n$ is an $\mathcal{E}$-bijection, it follows that $n^{-1} \mid H_{\alpha}=\left(n \mid H_{\alpha}\right)^{-1} \in$ $\mathcal{B}\left(H_{\alpha}\right)$. Taking into account that $n \geq 0$ and $\sigma(n)=\overline{\bigcup_{\alpha \in \Lambda} \sigma\left(n \mid H_{\alpha}\right)}$ (see Corollary 2.1), we deduce that $\sigma\left(n^{-1} \mid H_{\alpha}\right)=\sigma\left(n \mid H_{\alpha}\right)^{-1} \geq 0$; that is, $n^{-1} \mid H_{\alpha} \geq 0$. Moreover, $\left(m \mid H_{\alpha}\right)\left(n \mid H_{\alpha}\right)=\left(n \mid H_{\alpha}\right)\left(m \mid H_{\alpha}\right)$. Therefore $\left(n^{-1} m\right) \mid H_{\alpha}=\left(n^{-1} \mid H_{\alpha}\right)\left(m \mid H_{\alpha}\right)=$ $\left(m \mid H_{\alpha}\right)\left(n^{-1} \mid H_{\alpha}\right) \geq 0$; that is, $\left\langle\left(n^{-1} m\right) x, x\right\rangle \geq 0$ for all $x \in \mathcal{D}$. Since $n^{-1} m$ is bounded and $\mathcal{D}$ is dense, we derive that $n^{-1} m \geq 0$.

Now take $k \in M$. Then

$$
\begin{aligned}
\left(\left(n^{-1} m\right) k\right) \mid H_{\alpha} & =\left(\left(n^{-1} m\right) \mid H_{\alpha}\right)\left(k \mid H_{\alpha}\right)=\left(n^{-1} \mid H_{\alpha}\right)\left(m \mid H_{\alpha}\right)\left(k \mid H_{\alpha}\right) \\
& =\left(k\left(n^{-1} m\right)\right) \mid H_{\alpha}
\end{aligned}
$$

for all $\alpha \in \Lambda$. Whence $n^{-1} m \in M^{\prime}$. But $n^{-1} m \in C_{\mathcal{E}}^{*}(H)$; therefore $n^{-1} m \in M_{\mathcal{E}}^{\prime}$. Hence $M_{\mathcal{E}}^{\prime}$ is a $C^{*}$-algebra related to $M$ (see the passage after Definition [5.2).

8.2. The fractional space $M_{\mathcal{E}}^{\prime} / M$. Take $m \in M$. Consider the fractional space $C_{\mathcal{E}}^{*}(H) / m$ and its subspace $M_{\mathcal{E}}^{\prime} / m=\left\{T / m: T \in M_{\mathcal{E}}^{\prime}\right\}$. We set

$$
M_{\mathcal{E}}^{\prime} / M=\sum_{m \in M} M_{\mathcal{E}}^{\prime} / m,
$$

which is a subspace in $C_{\mathcal{E}}^{*}(H) / M$.

Lemma 8.2. The space $M_{\mathcal{E}}^{\prime} / m$ is a quantum system in $C_{\mathcal{E}}^{*}(\mathcal{D})$. Moreover, $M_{\mathcal{E}}^{\prime} / M$ is a fractional space in $C_{\mathcal{E}}^{*}(H) / M$. 
Proof. First, note that $1_{\mathcal{D}}=m / m \in M_{\mathcal{E}}^{\prime} / m$ and $1_{H} / m \in M_{\mathcal{E}}^{\prime} / m$. Further, each $T / m$ has the unbounded dual, namely, $T^{*} / m$. Indeed,

$$
\begin{aligned}
\langle(T / m) x, y\rangle & =\left\langle T(m \mid \mathcal{D})^{-1} x, y\right\rangle=\left\langle(m \mid \mathcal{D})^{-1} x, T^{*} y\right\rangle \\
& =\left\langle x,(m \mid \mathcal{D})^{-1} T^{*} y\right\rangle=\left\langle x, T^{*}(m \mid \mathcal{D})^{-1} y\right\rangle \\
& =\left\langle x,\left(T^{*} / m\right) y\right\rangle
\end{aligned}
$$

for all $x, y \in \mathcal{D}$. But $T^{*} / m \in C_{\mathcal{E}}^{*}(\mathcal{D})$. It follows that $(T / m)^{*}=T^{*} / m \in M_{\mathcal{E}}^{\prime} / m$, Proposition 3.1]. Thus $M_{\mathcal{E}}^{\prime} / m$ is a quantum system in $C_{\mathcal{E}}^{*}(\mathcal{D})$.

Now let $n \preceq m$ for some $n, m \in M$. If $T / n \in M_{\mathcal{E}}^{\prime} / n$, then $T / n=T n^{-1} m / m \in$ $C_{\mathcal{E}}^{*}(H) / m$. By Lemma 8.1, $n^{-1} m \in M_{\mathcal{E}}^{\prime}$. Therefore $T n^{-1} m \in M_{\mathcal{E}}^{\prime}$; that is, $T / n \in$ $M_{\mathcal{E}}^{\prime} / m$. Thus $M_{\mathcal{E}}^{\prime} / n \subseteq M_{\mathcal{E}}^{\prime} / m$. According to Definition 5.2, $M_{\mathcal{E}}^{\prime} / M$ is a fractional space in $C_{\mathcal{E}}^{*}(H) / M$.

Remark 8.1. Let $\Theta \subseteq C_{\mathcal{E}}^{*}(\mathcal{D})_{h}$ be a unital subset of symmetric operators, and let $M=\left\{D_{S}: S \in \Theta\right\}$ with $D_{S}=2-\bar{U}_{S}-\bar{U}_{S}^{*}$ (see (3.1)). Then $M$ is commutative if $\Theta$ is as well. Indeed, take $D_{S}, D_{T} \in M$ for some $T, S \in \Theta$. Note that

$$
D_{S} \mid H_{\alpha}=4\left(1+S^{2} \mid H_{\alpha}\right)^{-1} \in \mathcal{B}\left(H_{\alpha}\right)
$$

for all $\alpha \in \Lambda$, thanks to (3.3) (see the proof of Lemma 3.4). Therefore if $[S, T]=0$ in $C_{\mathcal{E}}^{*}(\mathcal{D})$, then $\left[S\left|H_{\alpha}, T\right| H_{\alpha}\right]=0$, which in turn implies that $\left[D_{S}\left|H_{\alpha}, D_{T}\right| H_{\alpha}\right]=0$ for all $\alpha \in \Lambda$. Further, note that

$$
\bar{U}_{S} \in M_{\mathcal{E}}^{\prime}
$$

Indeed, $\bar{U}_{S} \mid H_{\alpha}=r\left(S \mid H_{\alpha}\right)$ (see Remark 3.1), where $r(t)=(t-i) /(t+i), t \in \mathbb{R}$, is a rational function. Therefore

$$
\left[\bar{U}_{S}\left|H_{\alpha}, D_{T}\right| H_{\alpha}\right]=\left[r\left(S \mid H_{\alpha}\right), 4\left(1+T^{2} \mid H_{\alpha}\right)^{-1}\right]=0
$$

for all $\alpha \in \Lambda$. It follows that $\left[\bar{U}_{S}, D_{T}\right]=0$ for all $S, T \in \Theta$. But $\bar{U}_{S} \in \mathfrak{U}_{\mathcal{E}} \subseteq C_{\mathcal{E}}^{*}(H)$ (see Theorem 3.1), thereby $\bar{U}_{S} \in M^{\prime} \cap C_{\mathcal{E}}^{*}(H)=M_{\mathcal{E}}^{\prime}$.

Consider an $n$-tuple $S=\left(S_{1}, \ldots, S_{n}\right)$ of mutually commuting operators from the set $C_{\mathcal{E}}^{*}(\mathcal{D})_{h}$. Then we have a mutually commuting $n$-tuple $D_{S}=\left(D_{S_{1}}, \ldots, D_{S_{n}}\right)$ of bounded positive operators from $C_{\mathcal{E}}^{*}(H)$ (see Remark 8.1). For an $n$-tuple $\lambda=$ $\left(\lambda_{1}, \ldots, \lambda_{n}\right) \in \mathbb{Z}_{+}^{n}$ we set $D_{S}^{\lambda}=D_{S_{1}}^{\lambda_{1}} \cdots D_{S_{n}}^{\lambda_{n}} \in C_{\mathcal{E}}^{*}(H)$. Then

$$
\mathcal{S}=\left\{D_{S}^{\lambda}: \lambda \in \mathbb{Z}_{+}^{n}\right\}
$$

is a unital commutative set of denominators in $C_{\mathcal{E}}^{*}(H)$. Note that $D_{S}^{\lambda} \preceq D_{S}^{\mu}$ if $\lambda \leq \mu$ in $\mathbb{Z}_{+}^{n}$. Indeed,

$$
D_{S}^{-\lambda} D_{S}^{\mu}=\prod_{i=1}^{n} D_{S_{i}}^{-\lambda_{i}} D_{S_{i}}^{\mu_{i}}=\prod_{i=1}^{n} 4^{\mu_{i}-\lambda_{i}}\left(1+S_{i}^{2}\right)^{\lambda_{i}-\mu_{i}} \in \mathcal{B}(H)
$$

(see Remark 8.1). In particular, $\mathcal{S}$ is upward filtered; that is, for a couple $D_{S}^{\lambda}, D_{S}^{\mu}$ there corresponds $D_{S}^{\gamma}$ with $D_{S}^{\lambda} \preceq D_{S}^{\gamma}$ and $D_{S}^{\lambda} \preceq D_{S}^{\gamma}$. Just put $\gamma=\max \{\lambda, \mu\}$ in $\mathbb{Z}_{+}^{n}$. Thus

$$
\mathcal{S}_{\mathcal{E}}^{\prime}=\left\{T \in C_{\mathcal{E}}^{*}(H):\left[T, D_{S_{i}}\right]=0,1 \leq i \leq n\right\} .
$$


One can easily verify that

$$
\mathcal{S}_{\mathcal{E}}^{\prime}=\left\{T \in C_{\mathcal{E}}^{*}(H):\left[T\left|\mathcal{D}, S_{i}^{2}\right| \mathcal{D}\right]=0,1 \leq i \leq n\right\} .
$$

Moreover, as we have just confirmed in Remark 8.1, $\bar{U}_{S_{i}} \in \mathcal{S}_{\mathcal{E}}^{\prime}$ for all $i$.

Lemma 8.3. Let $M$ be a commutative set of denominators in $C_{\mathcal{E}}^{*}(H)$ and let $\alpha \in \Lambda$. Then $\left(M_{\mathcal{E}}^{\prime} / m\right)_{M, \alpha}^{+}=\left(M_{\mathcal{E}}^{\prime} / m\right)_{\alpha}^{+}$. In particular,

$$
\left(M_{\mathcal{E}}^{\prime} / M\right)_{\alpha}^{+}=\sum_{m \in M}\left(M_{\mathcal{E}}^{\prime} / m\right)_{\alpha}^{+},
$$

and if $M_{0} \subseteq M$ is a cofinal subset, then

$$
\left(M_{\mathcal{E}}^{\prime} / m\right)_{M_{0}, \alpha}^{+}=\left(M_{\mathcal{E}}^{\prime} / m\right)_{M, \alpha}^{+} \quad \text { and } \quad\left(M_{\mathcal{E}}^{\prime} / M\right)_{\alpha}^{+}=\left(M_{\mathcal{E}}^{\prime} / M_{0}\right)_{\alpha}^{+} .
$$

Proof. Evidently, $\left(M_{\mathcal{E}}^{\prime} / m\right)_{\alpha}^{+}=\left\{T / m \in M_{\mathcal{E}}^{\prime} / m: T \geq_{\alpha} 0\right\} \subseteq\left(M_{\mathcal{E}}^{\prime} / m\right)_{M, \alpha}^{+}$(see Remark 6.1). Take $b=T / m \in\left(M_{\mathcal{E}}^{\prime} / m\right)_{M, \alpha}^{+}$. Then $b=\sum_{i=1}^{k} T_{i} / n_{i}$ for some $T_{i} \in M_{\mathcal{E}}^{\prime}$, $T_{i} \geq_{\alpha} 0, n_{i} \in M, n_{i} \preceq m, 1 \leq i \leq k$ (see Subsection 6.2). By Lemma 8.1, $n_{i}^{-1} m \geq 0$ in the $C^{*}$-algebra $M_{\mathcal{E}}^{\prime}$. In particular, $n_{i}^{-1} m \geq_{\alpha} 0$. Moreover,

$$
\begin{aligned}
{\left[T_{i}\left|H_{\beta},\left(n_{i}^{-1} m\right)\right| H_{\beta}\right] } & =\left[T_{i} \mid H_{\beta},\left(n_{i} \mid H_{\beta}\right)^{-1}\left(m \mid H_{\beta}\right)\right] \\
& =\left[T_{i} \mid H_{\beta},\left(n_{i} \mid H_{\beta}\right)^{-1}\right]\left(m \mid H_{\beta}\right)+\left(n_{i} \mid H_{\beta}\right)^{-1}\left[T_{i} \mid H_{\beta},\left(m \mid H_{\beta}\right)\right] \\
& =0
\end{aligned}
$$

for all $\beta \in \Lambda$. Hence $\left[T_{i}, n_{i}^{-1} m\right]=0$ for all $i$. Therefore $T_{i}\left(n_{i}^{-1} m\right) \geq_{\alpha} 0$ for all $i$, which in turn implies that $S=\sum_{i=1}^{k} T_{i}\left(n_{i}^{-1} m\right) \geq_{\alpha} 0$. But $b=S / m$, so $b \in\left(M_{\mathcal{E}}^{\prime} / m\right)_{\alpha}^{+}$. Consequently, $\left(M_{\mathcal{E}}^{\prime} / m\right)_{M, \alpha}^{+}=\left(M_{\mathcal{E}}^{\prime} / m\right)_{\alpha}^{+}$, which in turn implies that $\left(M_{\mathcal{E}}^{\prime} / M\right)_{\alpha}^{+}=\sum_{m \in M}\left(M_{\mathcal{E}}^{\prime} / m\right)_{\alpha}^{+}$.

Finally, let $M_{0} \subseteq M$ be a cofinal subset. If $m \in M_{0}$, then (see Lemma 6.6)

$$
\left(M_{\mathcal{E}}^{\prime} / m\right)_{\alpha}^{+} \subseteq\left(M_{\mathcal{E}}^{\prime} / m\right)_{M_{0}, \alpha}^{+} \subseteq\left(M_{\mathcal{E}}^{\prime} / m\right)_{M, \alpha}^{+} \subseteq\left(M_{\mathcal{E}}^{\prime} / m\right)_{\alpha}^{+} ;
$$

that is, $\left(M_{\mathcal{E}}^{\prime} / m\right)_{\alpha}^{+}=\left(M_{\mathcal{E}}^{\prime} / m\right)_{M_{0}, \alpha}^{+}$. Whence $\left(M_{\mathcal{E}}^{\prime} / M\right)_{\alpha}^{+}=\left(M_{\mathcal{E}}^{\prime} / M_{0}\right)_{\alpha}^{+}$.

As follows from Lemma 8.3. all varieties of the fractional positivity (see Remark 6.1) that occurred in the noncommutative case are unified in the commutative one.

Now consider the matrix spaces over the fractional space $M_{\mathcal{E}}^{\prime} / M$. First note that $\mathbb{M}_{k}\left(M_{\mathcal{E}}^{\prime} / M\right)=\sum_{m \in M} \mathbb{M}_{k}\left(M_{\mathcal{E}}^{\prime} / m\right)$ for all $k$. Using (6.2) and (8.1), infer that

$$
\mathbb{M}_{k}\left(M_{\mathcal{E}}^{\prime} / m\right)=M_{\mathcal{E}^{k}}^{\prime} /\left(m \cdot 1_{H^{k}}\right),
$$

which in turn implies that

$$
\mathbb{M}_{k}\left(M_{\mathcal{E}}^{\prime} / M\right)=\sum_{m \in M} M_{\mathcal{E}^{k}}^{\prime} /\left(m \cdot 1_{H^{k}}\right)=M_{\mathcal{E}^{k}}^{\prime} / M^{k} .
$$

Due to Lemma 8.3 ,

$$
\left(M_{\mathcal{E}^{k}}^{\prime} /\left(m \cdot 1_{H^{k}}\right)\right)_{M^{k}, \alpha}^{+}=\left(M_{\mathcal{E}^{k}}^{\prime} /\left(m \cdot 1_{H^{k}}\right)\right)_{\alpha}^{+}=\left(M_{\mathcal{E}^{k}}^{\prime} /\left(m \cdot 1_{H^{k}}\right)\right)_{M_{0}^{k}, \alpha}^{+}
$$

for a cofinal subset $M_{0} \subseteq M$. Whence

$$
\left(M_{\mathcal{E}^{k}}^{\prime} / M^{k}\right)_{\alpha}^{+}=\sum_{m \in M^{k}}\left(M_{\mathcal{E}^{k}}^{\prime} /\left(m \cdot 1_{H^{k}}\right)\right)_{\alpha}^{+}=\left(M_{\mathcal{E}^{k}}^{\prime} / M_{0}^{k}\right)_{\alpha}^{+} .
$$


As above fix an $n$-tuple $S=\left(S_{1}, \ldots, S_{n}\right)$ of mutually commuting operators in $C_{\mathcal{E}}^{*}(\mathcal{D})_{h}$ and consider the commutative set $\mathcal{S}=\left\{D_{S}^{\lambda}: \lambda \in \mathbb{Z}_{+}^{n}\right\}$ of denominators in $C_{\mathcal{E}}^{*}(H)$. The unital $*$-subalgebra in $C_{\mathcal{E}}^{*}(\mathcal{D})$ generated by $S$ is denoted by $\mathcal{P}_{S}$. It is a quantum *-algebra in $C_{\mathcal{E}}^{*}(\mathcal{D})$ of all polynomials taken by $S$. If $v \in \mathbb{Z}_{+}^{n}$, then we have an unbounded operator

$$
D_{S}^{-v / 2}=\prod_{i=1}^{n} D_{S_{i}}^{-v_{i} / 2}=2^{-|v|} \prod_{i=1}^{n}\left(1_{\mathcal{D}}+S_{i}^{2}\right)^{v_{i} / 2} \in C_{\mathcal{E}}^{*}(\mathcal{D})
$$

where $\left(1_{\mathcal{D}}+S_{i}^{2}\right)^{v_{i} / 2} \mid H_{\alpha}=\left(\left(1+\left(S_{i} \mid H_{\alpha}\right)^{2}\right)^{v_{i}}\right)^{1 / 2}$ is the square root of a positive operator. For the fixed $\lambda \in \mathbb{Z}_{+}^{n}$ we consider the following subspace:

$$
\begin{aligned}
\mathcal{P}_{S, \lambda}=\operatorname{span}\left\{S^{v}\right. & =S_{1}^{v_{1}} \cdots S_{n}^{v_{n}}: D_{S}^{-v / 2} D_{S}^{\lambda} \\
& \left.=\prod_{i=1}^{n} 4^{\lambda_{i}-v_{i} / 2}\left(1_{\mathcal{D}}+S_{i}^{2}\right)^{v_{i} / 2-\lambda_{i}} \quad \text { is bounded }\right\} \subseteq \mathcal{P}_{S} .
\end{aligned}
$$

Note that if $v \leq 2 \lambda$, then each $\left(1_{\mathcal{D}}+S_{i}^{2}\right)^{v_{i} / 2-\lambda_{i}}$ is bounded. Therefore $D_{S}^{-v / 2} D_{S}^{\lambda}$ is bounded and therefore $S^{v} \in \mathcal{P}_{S, \lambda}$. Thus $\left\{S^{v}: v \leq 2 \lambda\right\} \subseteq \mathcal{P}_{S, \lambda}$. It follows that $\mathcal{P}_{S}=\sum_{\lambda} \mathcal{P}_{S, \lambda}$.

Lemma 8.4. The quantum *-algebra $\mathcal{P}_{S}=\sum_{\lambda} \mathcal{P}_{S, \lambda}$ is a fractional subspace in $\mathcal{S}_{\mathcal{E}}^{\prime} / \mathcal{S}$. Namely, $1_{H} / D_{S}^{\lambda} \in \mathcal{P}_{S, \lambda} \subseteq \mathcal{S}_{\mathcal{E}}^{\prime} / D_{S}^{\lambda}$ and $\mathcal{P}_{S, \lambda} \subseteq \mathcal{P}_{S, \mu}$ whenever $D_{S}^{\lambda} \preceq D_{S}^{\mu}$.

Proof. First note that being a quantum $*$-algebra, $\mathcal{P}_{S}$ is a fractional space thanks to Corollary 5.1. Let us prove that $\mathcal{P}_{S}=\sum_{\lambda} \mathcal{P}_{S, \lambda}$ is the fractional space expansion (see Definition 5.2). Note that

$$
\begin{aligned}
1_{H} / D_{S}^{\lambda} & =D_{S}^{-\lambda}=4^{-|\lambda|} \prod_{i=1}^{n}\left(1_{\mathcal{D}}+S^{2}\right)^{\lambda_{i}}=4^{-|\lambda|} \sum_{0 \leq q \leq \lambda}\left(\begin{array}{l}
\lambda \\
q
\end{array}\right) S^{2(\lambda-q)} \\
& \in \operatorname{span}\left\{S^{v}: v \leq 2 \lambda\right\} \subseteq \mathcal{P}_{S, \lambda}
\end{aligned}
$$

that is, $1_{H} / D_{S}^{\lambda} \in \mathcal{P}_{S, \lambda}$.

Now take $S^{v} \in \mathcal{P}_{S, \lambda}$. Then $S^{v} D^{\lambda}$ is bounded. Indeed, first prove that

$$
2^{|v|} D_{S}^{-v / 2} \geq_{\alpha} S^{v} \geq_{\alpha}-2^{|v|} D_{S}^{-v / 2}
$$

for all $\alpha \in \Lambda$. Note that the $C^{*}$-algebra $A_{\alpha}$ in $\mathcal{B}\left(H_{\alpha}\right)$ generated by the mutually commuting operators $S \mid H_{\alpha}=\left(S_{1}\left|H_{\alpha}, \ldots, S_{n}\right| H_{\alpha}\right)$ is a commutative $C^{*}$-algebra which is identified to within a $*$-isomorphism, with the algebra of all complex continuous functions $C\left(\sigma\left(S \mid H_{\alpha}\right)\right)$ on the character space $\sigma\left(S \mid H_{\alpha}\right)$ of $A_{\alpha}$. Let $t=\left(t_{1}, \ldots, t_{n}\right)$ be the real variables running in the spectrum $\sigma\left(S \mid H_{\alpha}\right)$. Since $\sigma\left(S \mid H_{\alpha}\right) \subseteq \prod_{i=1}^{n} \sigma\left(S_{i} \mid H_{\alpha}\right)$, it follows that $t_{i} \in \sigma\left(S_{i} \mid H_{\alpha}\right), 1 \leq i \leq n$. Moreover, $S^{v} \mid H_{\alpha}=\prod_{i=1}^{n} t_{i}^{v_{i}}$ and $2^{|v|} D_{S}^{-v / 2}=\prod_{i=1}^{n}\left(1+t_{i}^{2}\right)^{v_{i} / 2}$ in $C\left(\sigma\left(S \mid H_{\alpha}\right)\right)$. With $\left|\prod_{i=1}^{n} t_{i}^{v_{i}}\right| \leq$ $\prod_{i=1}^{n}\left(1+t_{i}^{2}\right)^{v_{i} / 2}$ in mind, we derive the inequality (8.2). Further, since $D_{S}^{\lambda} \geq 0$ and 
$D_{S}^{\lambda} \mid H_{\alpha}$ commutes with $D_{S}^{-v / 2} \mid H_{\alpha}$ and $S^{v} \mid H_{\alpha}$, we also deduce that $2^{|v|} D_{S}^{-v / 2} D_{S}^{\lambda} \geq_{\alpha}$ $S^{v} D_{S}^{\lambda} \geq_{\alpha}-2^{|v|} D_{S}^{-v / 2} D_{S}^{\lambda}$. In particular,

$$
\left\|S^{v} D_{S}^{\lambda}\left|H_{\alpha}\left\|\leq 2^{|v|}\right\| D_{S}^{-v / 2} D_{S}^{\lambda}\right| H_{\alpha}\right\| \leq 2^{|v|}\left\|D_{S}^{-v / 2} D_{S}^{\lambda}\right\|
$$

for all $\alpha \in \Lambda$. But $D_{S}^{-v / 2} D_{S}^{\lambda}$ is bounded, for $S^{v} \in \mathcal{P}_{S, \lambda}$. Consequently, $S^{v} D_{S}^{\lambda}$ is bounded.

In particular, $S^{\nu}=S^{\nu} D_{S}^{\lambda} / D_{S}^{\lambda} \in \mathcal{S}_{\mathcal{E}}^{\prime} / D_{S}^{\lambda}$. Thus $\mathcal{P}_{S, \lambda} \subseteq \mathcal{S}_{\mathcal{E}}^{\prime} / D_{S}^{\lambda}$ is a unital subspace.

Finally, $\mathcal{P}_{S, \lambda} \subseteq \mathcal{P}_{S, \mu}$ whenever $D_{S}^{\lambda} \preceq D_{S}^{\mu}$. Indeed, take $v \in \mathbb{Z}_{+}^{n}$ with $S^{v} \in \mathcal{P}_{S, \lambda}$. Then $D_{S}^{-v / 2} D_{S}^{\lambda}$ and $D_{S}^{-\lambda} D_{S}^{\mu}$ are bounded operators and

$$
D_{S}^{-v / 2} D_{S}^{\mu}=\left(D_{S}^{-v / 2} D_{S}^{\lambda}\right)\left(D_{S}^{-\lambda} D_{S}^{\mu}\right)
$$

turns out to be a bounded operator. By its very definition, $S^{v} \in \mathcal{P}_{S, \mu}$.

Proposition 8.1. Let $M \subseteq \mathfrak{M}_{\mathcal{E}}$ be a commutative set of denominators, $\mathcal{F}_{M}=$ $\sum_{m \in M} \mathcal{F}_{m} \subseteq M_{\mathcal{E}}^{\prime} / M$ a fractional space, and let $\phi: \mathcal{F}_{M} \rightarrow S F(\Delta)$ be an innerproduct mapping. Then $\phi$ is $\alpha$-admissible if and only if it is matrix $\alpha$-contractive.

Proof. By Lemma 7.7 each $\alpha$-admissible inner product mapping is matrix $\alpha$ contractive.

Now assume that $\phi: \mathcal{F}_{M} \rightarrow S F(\Delta)$ is a matrix $\alpha$-contractive inner-product mapping. By Lemma 7.6, there are matrix $\alpha$-positive and matrix $\alpha$-contractive mappings $\Psi_{m}: M_{\mathcal{E}}^{\prime} \rightarrow \mathcal{B}\left(\Delta_{m}\right)$ such that $\phi_{m}(T / m)(x, y)=\left\langle\Psi_{m}(T) x, y\right\rangle_{m}$ for all $T / m \in M_{\mathcal{E}}^{\prime} / m, m \in M_{0}$, where $M_{0} \subseteq M$ is a unital cofinal subset. If $n \preceq m$ for some $n, m \in M_{0}$, then $n^{-1} m \geq 0$ in the $C^{*}$-algebra $M_{\mathcal{E}}^{\prime}$ by virtue of Lemma 8.1 Take $T \in M_{\mathcal{E}}^{\prime}, T \geq_{\alpha} 0$. Then

$$
T\left(n^{-1} m\right) / m=T / n \in\left(M_{\mathcal{E}}^{\prime} / n\right)_{\alpha}^{+} \subseteq\left(M_{\mathcal{E}}^{\prime} / n\right)_{M, \alpha}^{+} \subseteq\left(M_{\mathcal{E}}^{\prime} / m\right)_{M, \alpha}^{+} \subseteq\left(M_{\mathcal{E}}^{\prime} / m\right)_{\alpha}^{+}
$$

due to Lemma 8.3. It follows that $T\left(n^{-1} m\right) \geq_{\alpha} 0$. Since $\mathbb{M}_{k}\left(M_{\mathcal{E}}^{\prime} / M\right)=M_{\mathcal{E}^{k}}^{\prime} / M^{k}$ and $\left(n^{-1} m\right) \cdot 1_{H^{k}} \geq 0$, we derive that $T\left(\left(n^{-1} m\right) \cdot 1_{H^{k}}\right) \geq_{\alpha} 0$ whenever $T \in M_{\mathcal{E}^{k}}^{\prime}$, $T \geq_{\alpha} 0$, on the same grounds. By assumption each $\Psi_{m}: M_{\mathcal{E}}^{\prime} \rightarrow \mathcal{B}\left(\Delta_{m}\right)$ is matrix $\alpha$-positive, therefore $\Psi_{m}^{(k)}\left(T\left(n^{-1} m \cdot 1_{H^{k}}\right)\right) \geq 0$. Thus $\Psi_{m}$ is $m$-fractionally matrix $\alpha$-positive (see Subsection 7.2). Therefore $\phi$ is $\alpha$-admissible (see Definition 7.4).

8.3. The quantum moment problem. Let us return to the quantum moment problem mentioned in the introduction. Fix an $n$-tuple $S=\left(S_{1}, \ldots, S_{n}\right)$ of mutually commuting symmetric operators in $C_{\mathcal{E}}^{*}(\mathcal{D})$ and consider the commutative set $\mathcal{S}=\left\{D_{S}^{\lambda}: \lambda \in \mathbb{Z}_{+}^{n}\right\}$ of denominators in $C_{\mathcal{E}}^{*}(H)$. According to Lemma 8.4, the polynomial *-algebra $\mathcal{P}_{S}$ generated by $S$ is a fractional space in $\mathcal{S}_{\mathcal{E}}^{\prime} / \mathcal{S}$. Consider an inner product space $\Delta$ whose completion is denoted by $K$, and also a unital linear mapping $\phi: \mathcal{P}_{S} \rightarrow S F(\Delta)$. We say that $\phi$ is an $H_{\alpha}$-moment form (or local moment form) if there is a quantum $\Delta$-measure $\mu: \mathcal{S}_{\mathcal{E}}^{\prime} / \mathcal{S} \rightarrow S F(\Delta)$ with support in $H_{\alpha}$ (see Definition 7.2) such that

$$
\phi(p(S))(x, x)=\mu_{x}(p(S)) \quad \text { for all } p(S) \in \mathcal{P}_{S} \text { and } x \in \Delta .
$$

In this case $\mu$ is called a representing quantum $\Delta$-measure for $\phi$. Replacing $\mu$ by a quantum $\Delta$-measure with support in $\mathcal{D}$, we can introduce a moment form. The moment forms can also be expressed in terms of quantum $\mathcal{B}(K)$-valued measures, as follows from Lemma 8.5 . 
Lemma 8.5. A unital linear mapping $\phi: \mathcal{P}_{S} \rightarrow S F(\Delta)$ is an $H_{\alpha}$-moment form iff there is a quantum $\mathcal{B}(K)$-valued measure $\Psi: \mathcal{S}_{\mathcal{E}}^{\prime} \rightarrow \mathcal{B}(K)$ with support in $H_{\alpha}$ such that $\phi(p(S))(x, x)=\widetilde{\mu}_{x}(p(S))$ for all $p(S) \in \mathcal{P}_{S}$ and $x \in \Delta$, where $\mu$ is a sesquilinear extension of $\Psi$.

Proof. It suffices to use Proposition 7.2 and Definition 7.3 ,

Now we prove the central result which solves the quantum moment problem.

Theorem 8.1. A unital linear mapping $\phi: \mathcal{P}_{S} \rightarrow S F(\Delta)$ is an $H_{\alpha}$-moment form if and only if $\phi$ is a matrix $\alpha$-contractive inner product mapping.

Proof. If $\phi$ is an $H_{\alpha}$-moment form, then there is a representing $\Delta$-measure $\mu$ : $\mathcal{S}_{\mathcal{E}}^{\prime} / \mathcal{S} \rightarrow S F(\Delta)$ with support in $H_{\alpha}$. By Lemma 7.4, $\mu$ is an inner product mapping, which is matrix $\alpha$-positive by virtue of Theorem 7.1. In particular, $\mu$ is $M_{0}$-fractionally $\alpha$-positive. Take $p(S) \in \mathcal{P}_{S}$. Using (7.2) and (8.3), we infer that

$$
\begin{aligned}
4 \mu(p(S))(x, y)= & 4 \mu_{x, y}(p(S))=\mu_{x+y}(p(S))-\mu_{x-y}(p(S)) \\
& +i \mu_{x+i y}(p(S))-i \mu_{x-i y}(p(S)) \\
= & \phi(p(S))(x+y, x+y)-\phi(p(S))(x-y, x-y) \\
+ & i \phi(p(S))(x+i y, x+i y)-i \phi(p(S))(x-i y, x-i y) \\
= & 4 \phi(p(S))(x, y) ;
\end{aligned}
$$

that is, $\mu(p(S))=\phi(p(S))$. Hence $\mu$ extends the mapping $\phi$. Further, by Corollary 7.1 .

$$
\begin{aligned}
\mu\left(1_{H} / m\right)(x, x) & =\sup \left\{|\mu(T / m)(x, x)|: T \in \mathcal{S}_{\mathcal{E}}^{\prime},\|T\|_{\alpha} \leq 1\right\}=\left\|\mu_{m, x}\right\|_{m, \alpha} \\
& \geq\left\|\phi_{m, x}\right\|_{m, \alpha} \geq \phi\left(1_{H} / m\right)(x, x)=\mu\left(1_{H} / m\right)(x, x)
\end{aligned}
$$

(see Remark 7.1); that is, $\left\|\mu_{m, x}\right\|_{m, \alpha}=\left\|\phi_{m, x}\right\|_{m, \alpha}$ for all $m \in \mathcal{S}, x \in \Delta$. Using Theorem 7.3, we infer that $\phi$ is $\alpha$-admissible, which in turn implies that $\phi$ is matrix $\alpha$-contractive thanks to Proposition 8.1 ,

Now assume that $\phi$ is a matrix $\alpha$-contractive inner product mapping. By Proposition 8.1. $\phi: \mathcal{P}_{S} \rightarrow S F(\Delta)$ is $\alpha$-admissible. Using Theorem 7.3, we deduce that $\phi$ extends up to a quantum $\Delta$-measure $\mu: \mathcal{S}_{\mathcal{E}}^{\prime} / \mathcal{S} \rightarrow S F(\Delta)$ with support in $H_{\alpha}$. It follows that $\mu_{x}(p(S))=\mu(p(S))(x, x)=\phi(p(S))(x, x)$ for all $p(S) \in \mathcal{P}_{S}$. Whence $\phi$ is $H_{\alpha}$-moment form.

Corollary 8.1. If $\mu: \mathcal{S}_{\mathcal{E}}^{\prime} / \mathcal{S} \rightarrow S F(\Delta)$ is a representing quantum $\Delta$-measure with support in $H_{\alpha}$ for an $H_{\alpha}$-moment form $\phi: \mathcal{P}_{S} \rightarrow S F(\Delta)$, then there exists a unique positive $\mathcal{B}(K)$-valued measure $E$ over all Borel sets in $\mathbb{R}^{n}$ whose support lies in $K_{\alpha}$ such that

$$
\phi(p(S))(x, x)=\int_{\mathbb{R}^{n}} p(t) d E_{x, x}(t) \quad \text { for all } p(S) \in \mathcal{P}_{S} \text { and } x \in \Delta .
$$

Conversely, each such spectral measure $E$ for an $H_{\alpha}$-moment form $\phi: \mathcal{P}_{S} \rightarrow$ $S F(\Delta)$ can be extended up to a quantum $\Delta$-measure $\mu$ with support in $H_{\alpha}$.

Proof. Let $\mathcal{C}_{S}$ be the unital $C^{*}$-algebra in $C_{\mathcal{E}}^{*}(H)$ generated by all unitaries $\bar{U}_{S_{i}}$, $1 \leq i \leq n$. Obviously, $\mathcal{C}_{S}$ is a $C^{*}$-algebra in $C_{\mathcal{E}}^{*}(H)$ related to $\mathcal{S}$ (see Remark 8.1). Moreover, $\mathcal{P}_{S}$ is a fractional subspace in $\mathcal{C}_{S} / \mathcal{S}$ (see the proof of Theorem 4.8 from 25]). The algebra $\mathcal{C}_{S}$ is a normal subalgebra in $\mathcal{B}(H)$ (actually in $\mathcal{S}_{\mathcal{E}}^{\prime}$ ), and all $H_{\alpha}$ 
are reducing subspaces for $\mathcal{C}_{S}$. Using Lemma 2.2, we derive that $\operatorname{Spec}\left(\mathcal{C}_{S}\right)$ is the closure of the union $\bigcup_{\alpha \in \Lambda} \operatorname{Spec}\left(\mathcal{C}_{S} \mid H_{\alpha}\right)$. Note that $\operatorname{Spec}\left(\mathcal{C}_{S}\right)$ is a compact subset in $\mathbb{T}^{n}$. If $\tau \in \operatorname{Spec}\left(\mathcal{C}_{S}\right)$ with $\tau\left(\bar{U}_{S_{i}}\right)=1$ for a certain $i$, then $\tau \notin \bigcup_{\alpha \in \Lambda} \operatorname{Spec}\left(\mathcal{C}_{S} \mid H_{\alpha}\right)$ by virtue of Proposition 3.1. Hence $\bigcup_{\alpha \in \Lambda} \operatorname{Spec}\left(\mathcal{C}_{S} \mid H_{\alpha}\right) \subseteq(\mathbb{T} \backslash\{1\})^{n}$, which means that $\left\{\operatorname{Spec}\left(\mathcal{C}_{S} \mid H_{\alpha}\right)\right\}$ are identified with an upward filtered family of compact sets $\left\{K_{\alpha}\right\}$ in $\mathbb{R}^{n}$ by means of the inverse Cayley transform. Further, the quantum *-algebra $\mathcal{P}_{S}$ is a normal algebra of unbounded operators thanks to Proposition 5.1. Furthermore, the fractional space $\mathcal{C}_{S} / \mathcal{S}$ is identified with the functional space $C\left(\operatorname{Spec}\left(\mathcal{C}_{S}\right)\right) / \mathcal{M}$ of fractions [25]. It follows that $\mathcal{P}_{S}$ can be identified with the $*-$ algebra $\mathcal{P}_{n}(X)$ of all polynomial functions on a certain (unbounded) subset $X \subseteq \mathbb{R}^{n}$ which is the union of its compact subsets $K_{\alpha}, \alpha \in \Lambda$.

Now comparing the results [2, Theorem 3.2] and Theorem 8.1, we obtain that if $\mu: \mathcal{S}_{\mathcal{E}}^{\prime} / \mathcal{S} \rightarrow S F(\Delta)$ is a representing quantum $\Delta$-measure with support in $H_{\alpha}$ for $\phi: \mathcal{P}_{S} \rightarrow S F(\Delta)$, then there exists a unique positive $\mathcal{B}(K)$-valued measure $E$ over all Borel sets in $K_{\alpha}$ such that $\mu_{x}(p(S))=\int_{K_{\alpha}} p(t) d E_{x, x}(t)$ for all $p(S) \in \mathcal{P}_{S}$ and $x \in \Delta$.

Finally, the extension of the spectral measure $E$ up to a quantum $\Delta$-measure $\mu$ is provided by the extension theorem, Theorem 7.3 .

Remark 8.2. It worth noting the global version of this assertion. Namely, if $\mu$ : $\mathcal{S}_{\mathcal{E}}^{\prime} / \mathcal{S} \rightarrow S F(\Delta)$ is a representing quantum $\Delta$-measure for a moment form $\phi$ : $\mathcal{P}_{S} \rightarrow S F(\Delta)$, then there exists a unique positive $\mathcal{B}(K)$-valued measure $E$ over all Borel sets in $\mathbb{R}^{n}$ such that $\phi(p(S))(x, x)=\int_{\mathbb{R}^{n}} p(t) d E_{x, x}(t), p(S) \in \mathcal{P}_{S}, x \in \Delta$. Conversely, each such spectral measure $E$ for a moment form $\phi: \mathcal{P}_{S} \rightarrow S F(\Delta)$ can be extended up to a quantum $\Delta$-measure $\mu$.

8.4. The moment form $\phi: \mathcal{P}_{S} \rightarrow S F(\mathcal{D})$. Now we apply Theorem 8.1 to a special example, that of $\Delta=\mathcal{D}$. For brevity, we consider the "global" moment form instead of the local one. Again fix an $n$-tuple $S=\left(S_{1}, \ldots, S_{n}\right)$ of mutually commuting symmetric operators in $C_{\mathcal{E}}^{*}(\mathcal{D})_{h}$ and consider the commutative set $\mathcal{S}=$ $\left\{D_{\mathcal{E}}^{\lambda}: \lambda \in \mathbb{Z}_{+}^{n}\right\}$ of denominators in $C_{\mathcal{E}}^{*}(H)$. By Lemma 8.4, the quantum *-algebra $\mathcal{P}_{S}$ is a fractional space in $\mathcal{S}_{\mathcal{E}}^{\prime} / \mathcal{S}$. Put $\Delta=\mathcal{D}$ and consider the following linear mapping:

$$
\phi: \mathcal{P}_{S} \rightarrow S F(\mathcal{D}), \quad \phi\left(S^{v}\right)(x, y)=\left\langle S^{v} x, y\right\rangle,
$$

where $v \in \mathbb{Z}_{+}^{n}$.

Lemma 8.6. The linear mapping $\phi: \mathcal{P}_{S} \rightarrow S F(\mathcal{D})$ is a matrix contractive inner product mapping. In particular, $\phi$ is a moment form.

Proof. First note that $\phi$ is an inner product mapping (see Definition 7.1). If $x \in$ $\mathcal{D} \backslash\{0\}$ and $\lambda \in \mathbb{Z}_{+}^{n}$, then we have

$$
\begin{aligned}
\phi\left(1 / D_{S}^{\lambda}\right)(x, x) & =\left\langle\prod_{i=1}^{n} 4^{-\lambda_{i}}\left(1+S_{i}^{2}\right)^{\lambda_{i}} x, x\right\rangle=4^{-|\lambda|}\left\langle\prod_{i=1}^{n}\left(1+S_{i}^{2}\right)^{\lambda_{i}} x, x\right\rangle \\
& \geq 4^{-|\lambda|}\langle x, x\rangle>0 .
\end{aligned}
$$

Further, put $\langle x, y\rangle_{\lambda}=\left\langle D_{S}^{-\lambda} x, y\right\rangle, x, y \in \mathcal{D}$, and take $T=\left[T_{i j}\right] \in \mathbb{M}_{k}\left(\mathcal{S}_{\mathcal{E}}^{\prime}\right)=\mathcal{S}_{\mathcal{E}^{k}}^{\prime}$ (see (8.1)). Then $\left[T\left|H_{\alpha}^{k},\left(D_{S}^{\lambda} \cdot 1_{H^{k}}\right)\right| H_{\alpha}^{k}\right]=0$ for each $\alpha$. It follows that

$$
\left\|T^{*} T\right\|\left(D_{S}^{-\lambda} \cdot 1_{H^{k}}\right) \geq_{\alpha} T^{*} T\left(D_{S}^{-\lambda} \cdot 1_{H^{k}}\right) \geq_{\alpha} 0 .
$$


Confirm that $0 \leq T^{*} T \leq\left\|T^{*} T\right\| 1_{H^{k}}$. Consequently,

$$
\left\langle T^{*} T\left(D_{S}^{-\lambda} \cdot 1_{H^{k}}\right) x, x\right\rangle \leq\left\|T^{*} T\right\|\left\langle\left(D_{S}^{-\lambda} \cdot 1_{H^{k}}\right) x, x\right\rangle \text { for all } x \in \mathcal{D}^{k} .
$$

Then

$$
\begin{aligned}
\left(\|T x\|_{\lambda}^{(k)}\right)^{2} & =\langle T x, T x\rangle_{\lambda}^{(k)}=\left\langle T^{*} T\left(D_{S}^{-\lambda} \cdot 1_{H^{k}}\right) x, x\right\rangle^{(k)} \\
& \leq\left\|T^{*} T\right\|\left\langle\left(D_{S}^{-\lambda} \cdot 1_{H^{k}}\right) x, x\right\rangle=\|T\|^{2}\left(\|x\|_{\lambda}^{(k)}\right)^{2}
\end{aligned}
$$

for all $x \in \mathcal{D}^{(k)}$. Hence

$$
\begin{aligned}
\left|\phi^{(k)}\left(T /\left(D_{S}^{\lambda} \cdot 1_{H^{k}}\right)\right)(x, y)\right| & =\left|\left\langle T\left(D_{S}^{-\lambda} \cdot 1_{H^{k}}\right) x, y\right\rangle\right|=\left|\left\langle\left(D_{S}^{-\lambda} \cdot 1_{H^{k}}\right) T x, y\right\rangle\right| \\
& =\left|\langle T x, y\rangle_{\lambda}^{(k)}\right| \leq\|T x\|_{\lambda}^{(k)}\|y\|_{\lambda}^{(k)} \leq\|T\|\|x\|_{\lambda}^{(k)}\|y\|_{\lambda}^{(k)},
\end{aligned}
$$

which in turn implies that $\left\|\phi^{(k)}\left(T /\left(D_{S}^{\lambda} \cdot 1_{H^{k}}\right)\right)\right\|_{\lambda}^{(k)} \leq\|T\|=\left\|T /\left(D_{S}^{\lambda} \cdot 1_{H^{k}}\right)\right\|_{\lambda}^{(k)}$ and $\left\|\phi_{\lambda}^{(k)}\right\|_{\lambda}^{(k)}=\sup \left\{\left\|\phi_{\lambda}^{(k)}(b)\right\|_{\lambda}^{(k)}:\|b\|_{\lambda}^{(k)} \leq 1\right\} \leq 1$ for all $\lambda$. That is, $\phi$ is a matrix contractive inner-product mapping. By Theorem 8.1, $\phi$ is a moment form.

\section{The NONCOMMUTing VARIABLES}

A noncommutative version of the moment form $\phi: \mathcal{P}_{S} \rightarrow S F(\Delta)$ from the previous section has a more complicated structure. In this final section we demonstrate how the general framework of the quantum moment problem developed in the previous sections can be used for a certain class of noncommutative tuples of symmetric operators from the algebra $C_{\mathcal{E}}^{*}(\mathcal{D})$.

Let $S=\left(S_{1}, \ldots, S_{n}\right)$ be an $n$-tuple of operators in $C_{\mathcal{E}}^{*}(\mathcal{D})_{h}$ and let $\mathcal{P}_{S}$ be a unital associative subalgebra in $C_{\mathcal{E}}^{*}(\mathcal{D})$ generated by $S$. Thus $\mathcal{P}_{S}$ is a quantum $*$-algebra. For each tuple $J=\left(j_{1}, \ldots, j_{k}\right), 1 \leq j_{s} \leq n$, we use $S_{J, \epsilon}$ to indicate the "real" noncommutative monomial $\operatorname{Re}\left((-i)^{\epsilon} S_{J}\right)$ in $\mathcal{P}_{S}$, where $\epsilon=0,1$ and $S_{J}=S_{j_{1}} \cdots S_{j_{k}}$. Since each element in $\mathcal{P}_{S}$ has unique expansion by means of its real and imaginary parts, we derive that the linear space $\mathcal{P}_{S}$ is generated by the monomials $S_{J, \epsilon}$, where $\epsilon=0,1$ and $J$ runs over all tuples in elements of the finite set $F_{n}=\{1,2, \ldots, n\}$. For the fixed tuple $J=\left(j_{1}, \ldots, j_{k}\right)$ we define its conjugate $J^{*}$ as $J^{*}=\left(j_{k}, \ldots, j_{1}\right)$. Evidently, $S_{J}^{*}=S_{J^{*}}$. Further, for the tuples $J=\left(j_{1}, \ldots, j_{k}\right)$ and $K=\left(k_{1}, \ldots, k_{s}\right)$ we put $J \cup K=\left(j_{1}, \ldots, j_{k}, k_{1}, \ldots, k_{s}\right)$. Evidently, $(J \cup K)^{*}=K^{*} \cup J^{*}$. Note that

$$
\begin{aligned}
S_{J, \epsilon}^{2} & =\operatorname{Re}\left((-i)^{\epsilon} S_{J}\right)^{2}=4^{-1}\left((-i)^{\epsilon} S_{J}+i^{\epsilon} S_{J}^{*}\right)^{2} \\
& =4^{-1}\left((-1)^{\epsilon} S_{J}^{2}+(-1)^{\epsilon} S_{J}^{* 2}+S_{J} S_{J}^{*}+S_{J}^{*} S_{J}\right) \\
& =4^{-1}\left((-1)^{\epsilon} S_{J \cup J}+(-1)^{\epsilon} S_{J^{*} \cup J^{*}}+S_{J \cup J^{*}}+S_{J^{*} \cup J}\right) \\
& =4^{-1}\left((-1)^{\epsilon}\left(S_{J \cup J}+S_{(J \cup J)^{*}}\right)+\left(S_{J \cup J^{*}}+S_{\left(J \cup J^{*}\right)^{*}}\right)\right) \\
& =4^{-1}\left((-1)^{\epsilon} 2 \operatorname{Re}\left(S_{J \cup J}\right)+2 \operatorname{Re}\left(S_{J \cup J^{*}}\right)\right)=2^{-1}\left((-1)^{\epsilon} S_{J \cup J, 0}+S_{J \cup J^{*}, 0}\right) .
\end{aligned}
$$

Thus in the noncommutative case a simple algebraic operation over the monomials $S_{J, \epsilon}$ would change the relevant tuples $J$ significantly. So, it is not possible (except in some special cases) to obtain a similar decomposition for the algebra $\mathcal{P}_{S}$ as in the commutative case proposed in Lemma 8.4. Nonetheless, some decomposition exists in the general case. 
Consider the real subspace $P_{S}$ in $\mathcal{P}_{S}$ generated by these monomials. Thus each element $a \in P_{S}$ is a "real" linear combination $a=\sum_{J, \epsilon} r_{J, \epsilon} S_{J, \epsilon}, r_{J, \epsilon} \in \mathbb{R}$. In particular, $P_{S} \subseteq C_{\mathcal{E}}^{*}(\mathcal{D})_{h}$.

Let us introduce the set $M=\left\{D_{a}: a \in P_{S}\right\}$ of positive denominators in $C_{\mathcal{E}}^{*}(H)$, where

$$
D_{a}=2-\bar{U}_{a}-\bar{U}_{a}^{*}
$$

Since $D_{a} \mid H_{\alpha}=4\left(1+a^{2} \mid H_{\alpha}\right)^{-1}\left(\right.$ see $(\underline{3.3})$ ), it follows that $\left(1+a^{2}\right)^{-1} \in C_{\mathcal{E}}^{*}(H)$ for each $a \in P_{S}$.

Definition 9.1. We say that $S=\left(S_{1}, \ldots, S_{n}\right)$ is an admissible $n$-tuple of operators in $C_{\mathcal{E}}^{*}(\mathcal{D})_{h}$ if for each couple $a, b \in P_{S}$ there is $c \in P_{S}$ such that the operators $a^{2}\left(1+c^{2}\right)^{-1}$ and $b^{2}\left(1+c^{2}\right)^{-1}$ are bounded.

If $S$ is an $n$-tuple of mutually commuting operators in $C_{\mathcal{E}}^{*}(\mathcal{D})_{h}$, then it is admissible. Indeed, in this case $S_{J, \epsilon}=S_{J}$, and $P_{S}$ is just the real linear space generated by the monomials $S_{J}$. Since $a, b \in P_{S}$ are commuting operators in $C_{\mathcal{E}}^{*}(\mathcal{D})_{h}$, it suffices to set $c=a^{2}+b^{2}$. As an example of a noncommutative case, one may consider an $n$-tuple $S$ for which all monomials $S_{J, \epsilon}$ of length at least $k$ are commuting. This type of example can be constructed in some formal way, taking an appropriate quotient of the relevant free algebra. In order to satisfy the conditions of Definition 9.1 in this case, it makes sense to consider polynomials $a=p(S), b=q(S) \in P_{S}$ without free terms. Then $a^{2^{k}}$ and $b^{2^{k}}$ are commuting for a certain $k$. It follows that $a^{2^{k}}\left(1+c^{2}\right)^{-1}$ and $b^{2^{k}}\left(1+c^{2}\right)^{-1}$ are bounded whenever $c=a^{2^{k+1}}+b^{2^{k+1}}$. But $a^{2}\left(1+a^{2^{k}}\right)^{-1}$ and $b^{2}\left(1+b^{2^{k}}\right)^{-1}$ are bounded too. Consequently, $a^{2}\left(1+c^{2}\right)^{-1}$ and $b^{2}\left(1+c^{2}\right)^{-1}$ are bounded.

Lemma 9.1. Let $S=\left(S_{1}, \ldots, S_{n}\right)$ be an $n$-tuple of operators in $C_{\mathcal{E}}^{*}(\mathcal{D})_{h}$. Then $S$ is admissible iff the set $M=\left\{D_{a}: a \in P_{S}\right\}$ of denominators is upward filtered. Moreover, the subset $M_{k}=\left\{D_{a}: a \in P_{S}^{(k)}\right\} \subseteq M$ is a unital cofinal subset, where $P_{S}^{(k)}=\left\{a^{2^{k}}: a \in P_{S}\right\}, k \in \mathbb{N}$.

Proof. By its very definition, $D_{a} \preceq D_{c}$ means that the operator $D_{a}^{-1} D_{c}$ is bounded. But $D_{a}^{-1} D_{c}=\left(1+a^{2}\right)\left(1+c^{2}\right)^{-1}$, thanks to (3.3). Thereby, the operator $D_{a}^{-1} D_{c}$ is bounded iff $a^{2}\left(1+c^{2}\right)^{-1}$ is as well. In particular, for each couple $a, b \in P_{S}$ there corresponds $c \in P_{S}$ such that $D_{a} \preceq D_{c}$ and $D_{b} \preceq D_{c}$.

Further, as we have shown above, $a^{2}\left(1+a^{2^{k+1}}\right)^{-1}$ is bounded for each $a \in P_{S}$. In particular, $D_{a} \preceq D_{b}$, where $b=a^{2^{k}} \in P_{S}^{(k)}$. Consequently, $M_{k} \subseteq M$ is a cofinal subset.

We write $a \preceq b$ for some $a, b \in P_{S}$, if $D_{a} \preceq D_{b}$; that is, $D_{a}^{-1} D_{b}$ has the bounded linear extension denoted by $D_{a b}$. Thus $a \preceq b$ iff the operator $a^{2}\left(1+b^{2}\right)^{-1}$ is bounded thanks to Lemma 9.1 .

Now we put $\mathcal{P}_{a}=\mathcal{P}_{S} \cap\left(C_{\mathcal{E}}^{*}(H) / D_{a}\right)$. Then $1_{H} / D_{a}=D_{a}^{-1}=4^{-1}\left(1_{\mathcal{D}}+a^{2}\right) \in$ $\mathcal{P}_{S}$. Using Corollary 5.1, we deduce that $\mathcal{P}_{S}=\sum_{a \in P_{S}} \mathcal{P}_{a}$ is a fractional space. 
Now take $n=D_{a}, m=D_{b}$, such that $n \preceq m$. Then $1 / n=D_{a}^{-1}=4^{-1}\left(1_{\mathcal{D}}+a^{2}\right)$, $1 / m=D_{b}^{-1}=4^{-1}\left(1_{\mathcal{D}}+b^{2}\right)$ and $m / n^{2}=4^{-1}\left(1_{\mathcal{D}}+b^{2}\right)^{-1}\left(1_{\mathcal{D}}+a^{2}\right)^{2}$. We set

$$
R_{a b}=\left(1_{\mathcal{D}}+b^{2}\right)^{-1}\left(1_{\mathcal{D}}+a^{2}\right)^{2} \in C_{\mathcal{E}}^{*}(\mathcal{D})
$$

whenever $a \preceq b$. Let us introduce the $*$-linear subspace $\mathcal{R}_{S}$ in $C_{\mathcal{E}}^{*}(\mathcal{D})$ generated by all $a$ and $R_{a b}$. Obviously, $\mathcal{P}_{S} \subseteq \mathcal{R}_{S}$. If $\mathcal{R}_{a}=\mathcal{R}_{S} \cap\left(C_{\mathcal{E}}^{*}(H) / D_{a}\right)$, then $1_{H} / D_{a} \in \mathcal{P}_{a} \subseteq \mathcal{R}_{a}$ and $\mathcal{R}_{a} \subseteq \mathcal{R}_{b}$ whenever $a \preceq b$. Indeed, the latter relation between polynomials implies that $D_{a} \preceq D_{b}$ (see Lemma 9.1). Then $C_{\mathcal{E}}^{*}(H) / D_{a} \subseteq C_{\mathcal{E}}^{*}(H) / D_{b}$ (see Lemma 5.2), which in turn implies that $\mathcal{R}_{a} \subseteq \mathcal{R}_{b}$. Thus $\mathcal{R}_{S}=\sum_{a \in P_{S}} \mathcal{R}_{a}$ is a fractional space in $C_{\mathcal{E}}^{*}(\mathcal{D})$ (see Definition 5.2). Furthermore, $R_{a b}=4 m / n^{2}=4\left(m n^{-1}\right) / n=4\left(n^{-1} m\right)^{*} / n \in \mathcal{R}_{S} \cap\left(C_{\mathcal{E}}^{*}(H) / D_{a}\right)=\mathcal{R}_{a}$.

Each unital $\alpha$-contractive linear functional $\phi: \mathcal{R}_{S} \rightarrow \mathbb{C}$ is uniquely determined by the numbers $\gamma_{a}=\phi(a)$ and $\gamma_{a b}=\phi\left(R_{a b}\right)$ with $a \preceq b$. As in the previous section $\phi: \mathcal{R}_{S} \rightarrow \mathbb{C}$ is said to be an $H_{\alpha}$-moment form if there is a quantum $\mathbb{C}$-measure $\mu: C_{\mathcal{E}}^{*}(H) / M \rightarrow \mathbb{C}$ with support in $H_{\alpha}$ (see Subsection 7.3) such that

$$
\mu(a)=\gamma_{a} \quad \text { and } \quad \mu\left(R_{a b}\right)=\gamma_{a b}, \quad a \preceq b .
$$

In this case $\mu$ is called a representing (scalar) measure for $\phi$.

Theorem 9.1. If $\phi: \mathcal{R}_{S} \rightarrow \mathbb{C}$ is a unital $\alpha$-contractive functional such that

$$
\phi\left(\left(1_{\mathcal{D}}+b^{2}\right)^{-1}\left(1_{\mathcal{D}}+a^{2}\right)^{2}\right)=\frac{\left(1+\phi\left(a^{2}\right)\right)^{2}}{1+\phi\left(b^{2}\right)}
$$

for all $a, b, a \preceq b$, then $\phi$ is an $H_{\alpha}$-moment form.

Proof. Since $\mathcal{R}_{S}$ is a quantum system in $C_{\mathcal{E}}^{*}(\mathcal{D})$, it follows that $\phi$ is matrix $\alpha$ positive. In particular, $\phi\left(a^{2}\right) \geq 0$. Thereby $\phi\left(1_{H} / D_{a}\right)>0$ for all $a \in P_{S}$. Hence $\phi$ is an inner product mapping. By assumption

$$
\phi\left(1 / D_{a}\right)^{2}=\phi\left(1 / D_{b}\right) \phi\left(D_{b} / D_{a}^{2}\right)
$$

whenever $D_{a} \preceq D_{b}$. Using Corollary 7.2 , we infer that $\phi$ is $\alpha$-admissible. By Theorem 7.3 the mapping $\phi$ extends up to a quantum scalar measure $\mu: C_{\mathcal{E}}^{*}(H) / M \rightarrow \mathbb{C}$ with support in $H_{\alpha}$. Whence $\phi$ is an $H_{\alpha}$-moment form.

Remark 9.1. One may restrict the assumptions demanded in Theorem 9.1 using the cofinal subset $M_{k} \subseteq M$ proposed in Lemma 9.1.

\section{ACKNOWLEDGEMENT}

The author wishes to thank F.-H. Vasilescu for useful discussions of the present work and for sending the papers 2], 24] and 25. The author also thanks the referee(s) for the attentive reading and valuable comments, which improved the paper significantly.

\section{REFERENCES}

[1] Alcantara-Bode J., Yngvason J., Algebraic quantum field theory and noncommutative moment problems I, Ann. Inst. Henri Poincare 48 (2) 147-159 (1988). MR952659 (90e:46062)

[2] Albrecht E., Vasilescu F.-H., Unbounded extensions and operator moment problems Preprint (2004).

[3] Bourbaki N., General topology, the main structures, Nauka, Moscow (1968). MR0244925 (39:6238)

[4] Bourbaki N., Spectral Theory, MIR, Moscow (1972). MR0358350 (50:10816) 
[5] Doherty A. C., Liang Y.-C., Toner B., Wehner S., The quantum moment problem and bounds on entagled multi-prover games, IEEE, Comp. Compl. 199-210 (2008). MR2513501 (2010e:81058)

[6] Dosiev A. A., Cartan-Slodkowski spectra, splitting elements and noncommutative spectral mapping theorems, J. Funct. Anal. 230 446-493 (2006). MR2186220 (2006g:47010)

[7] Dosiev A. A., A representation theorem for quantum spaces, Funct. Anal. its Appl. 41 (4) 306-310 (2007). MR2411608

[8] Dosiev A. A., Quantized moment problem, C. R. Acad. Sci. Paris 344 (I) 627-630 (2007). MR2334073 (2008f:46086)

[9] Dosiev A. A., Local operator spaces, unbounded operators and multinormed $C^{*}$-algebras, J. Funct. Anal. 255 1724-1760 (2008). MR2442081

[10] Conway J. B., A course in Operator Theory, Amer. Math. Soc. 21 (2000). MR1721402 (2001d:47001)

[11] Effros E. G., Advances in quantized functional analysis, Proc. ICM Berkeley, 1986. MR.934293 (89e:46064)

[12] Effros E. G., Ruan Z.-J., Operator spaces, London Math. Soc. Clarendon Press-Oxford (2000). MR1793753 (2002a:46082)

[13] Effros E. G., Webster C., Operator analogues of locally convex spaces, Operator Algebras and Applications (Samos 1996), (NATO Adv. Sci. Inst. Ser. C Math. Phys. Sci., 495), Kluwer (1997). MR 1462680 (99b:46081)

[14] Helemskii A.Ya., Tensor Products in Quantum Functional Analysis: the Non-Matricial Approach, Topological algebras and applications, 199-223, Contemp. Math., 427, Amer. Math. Soc., Providence, RI (2007). MR2326357 (2008f:46075)

[15] Helemskii A.Ya., Banach and polinormed algebras: General theory, representations, homology, Nauka, Moscow (1989). MR1031991(91h:46001)

[16] Helemskii A.Ya., Lectures and exercises on functional analysis, 233 Amer. Math. Soc., Providence, RI (2006). MR2248303 (2007k:46001)

[17] Kutateladze S. S., Fundamentals of functional analysis, Kluwer Acad. Publ. 12 (1995). MR.1384068 (97a:46001)

[18] Kiukas J., Moment Operators of Observables in Quantum Mechanics, with Applications to Quantization and Homodyne Detection, Turun Y. J. Annl. Univ. T. 380, (2007).

[19] Mokni H., Positive functionals on some spaces of fractions, Positivity 11 (2007), 417-432. MR2336206 (2009c:46055)

[20] Rudin W. Functional Analysis, Intern. Ser. Pure and Appl. Math., McGraw-Hill (1991). MR1157815 (92k:46001)

[21] Schmudgen K., Unbounded operator algebras and representation theory, Operator Theory: Advances and Appl. 37, Birkhauser Verlag, Basel/Boston/Berlin (1990). MR.1056697 (91f:47062)

[22] Turovskii Yu. V., On spectral properties of some Lie algebras and spectral radius of subsets of a Banach algebra, Sb. Spekt. Teor. Operator i Prilozhen. Baku ELM (1985) 144-181. MR849356 (87k:46102)

[23] Vasilescu F.-H., Operator moment problems in unbounded sets, Operator Theory: Advances and Applications (127) 613-638, Birkhauser Verlag, Basel, 2001. MR1902905 (2003d:47018)

[24] Vasilescu F.-H., Spaces of fractions and positive functionals, Math. Scand. 96 257-279 (2005). MR2153414 (2007b:47040)

[25] Vasilescu F.-H., Unbounded normal algebras and spaces of fractions, Proceedings of the Conference "Operator Theory, System Theory and Scattering Theory: Multidimensional Generalizations and Related Topics", Beer-Sheva (2005).

[26] Webster C., Local operator spaces and applications, Ph.D. University of California, Los Angeles (1997).

Department of Mathematics, Middle East Technical University, Northern Cyprus Campus, Guzelyurt, KKTC via Mersin 10, Turkey

E-mail address: dosiev@yahoo.com

E-mail address: dosiev@metu.edu.tr 